\title{
Illuminating Stannylation
}

Kyoka Sakamoto, ${ }^{\dagger}$ Yuki Nagashima, ${ }^{*, \dagger, \ddagger}$ Chao Wang, ${ }^{\dagger}$ Kazunori Miyamoto, ${ }^{\dagger}$ Ken

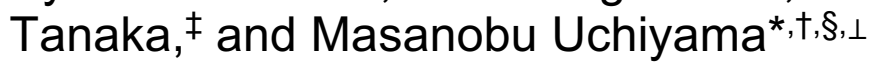

${ }^{\dagger}$ Graduate School of Pharmaceutical Sciences, The University of Tokyo, 7-3-1 Hongo, Bunkyo-ku, Tokyo 113-0033, Japan

¥Department of Chemical Science and Engineering, Tokyo Institute of Technology, O-okayama, Meguro-ku, Tokyo 1528550, Japan

$\S$ Cluster for Pioneering Research (CPR), RIKEN, 2-1 Hirosawa, Wako, Saitama 351-0198, Japan

$\perp$ Research Initiative for Supra-Materials (RISM), Shinshu University, 3-15-1 Tokida, Ueda, Nagano 386-8567, Japan

\section{Table of Contents}

1. General

S-2

2. Experimental Section

2.1. Stannylation of Various Alkynes (Table 2)

2.2. Dehalostannylation of Aryl Halides (Table 4)

S-12

2.3. Sequential Stannylation/Stille Coupling Processes (Scheme 2)

3. Computational Details

4. References

S-23

5. Copies of NMR Spectra 


\section{General}

\section{Instrumentation.}

NMR spectra were obtained on a BRUKER AVANCE III HD 500 spectrometer. Chemical shifts are expressed in

$\delta \quad(\mathrm{ppm})$ values and coupling constants are expressed in hertz $(\mathrm{Hz})$. All ${ }^{1} \mathrm{H}$ NMR experiments are reported in $\delta$ units, parts per million (ppm), and were measured relative to the signals for residual chloroform (7.26 ppm). All ${ }^{13} \mathrm{C}$ NMR spectra are reported in $\mathrm{ppm}$ relative to deuterochloroform $(77.16 \mathrm{ppm})$ and were obtained with ${ }^{1} \mathrm{H}$ decoupling. The following abbreviations are used: $\mathrm{s}=$ singlet, $\mathrm{d}=$ doublet, $\mathrm{t}=$ triplet, $\mathrm{m}=$ multiplet, and $\mathrm{bs}=$ broad singlet. EI mass spectra with gas clomatography were measured on an Agilent 5977B spectrometer. All previously unreported compounds were additionally characterized by high-resolution mass spectrometry (HRMS). ESI mass spectra (HRMS analyses) were measured on a Bruker micrOTOF-II spectrometer. IR spectra were obtained on a JASCO FR/IR-4700 spectrometer. Melting points were determined with a Yanaco micro melting point apparatus.

\section{Materials.}

Unless otherwise noted, materials were purchased from Aldrich Inc., Wako Pure Chemical Industries, Ltd., Tokyo Kasei Co., and other commercial suppliers and were used after appropriate purification. Anhydrous solvents were purchased from Kanto Chemical Co. Ltd. Trimethyl(tributylstannyl)silane (2) was purchased from Aldrich Inc., and stored under argon atmosphere. All other chemicals were of reagent grade and used as received. Air- and moisture-sensitive manipulations were performed with standard Schlenk techniques under argon atmosphere. Photoreactions were performed in a Schlenck tube using a blue LED light (Kessil A160WE TUNA Blue). Normalphase column chromatography was performed with YAMAZEN YFLC AI-580.

NOTE: Normal-phase column chromatography was performed within 15 minutes, since many of stannylated products $(\mathbf{3}, \mathbf{5}$ and $\mathbf{1 2})$ are not so stable on silica gel. 


\section{Experimental Section}

\subsection{Stannylation of Various Alkynes (Table 2)}

Typical Procedure A:

(E)-tributyl(oct-1-en-1-yl)stannane (3a)

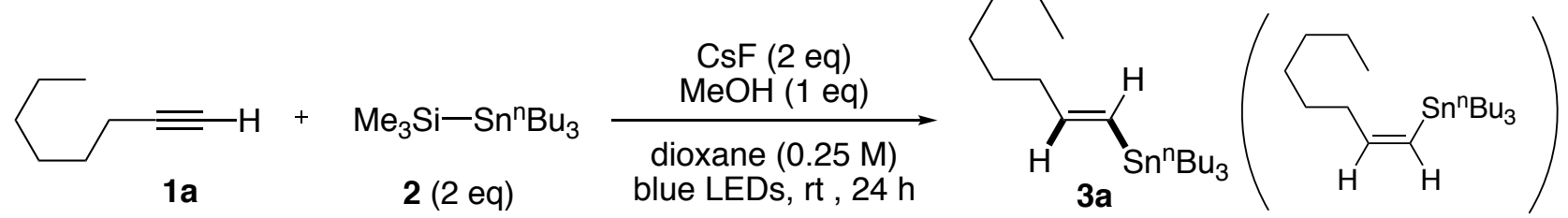

CsF (76 mg, $0.50 \mathrm{mmol}$ ) was charged in a dried tube at argon glovebox, and dissolved in dry dioxane $(0.5 \mathrm{~mL})$. To the mixture was successively added $\mathrm{MeOH}(8.01 \mathrm{mg}, 0.250 \mathrm{mmol})$, trimethyl(tributylstannyl)silane $(2,182 \mathrm{mg}$, $0.50 \mathrm{mmol})$ and 1-octyne (1a, $27.6 \mathrm{mg}, 0.25 \mathrm{mmol})$ at room temperature. After the reaction tube was sealed and irradiated with a blue LED lamp, the solution was stirred for $24 \mathrm{~h}$ at room temperature. The reaction was quenched by $\mathrm{H}_{2} \mathrm{O}(1 \mathrm{~mL})$, and then extracted with AcOEt $(3 \mathrm{~mL} \times 3)$. The AcOEt layers were combined and dried over $\mathrm{MgSO}_{4}$, and the solvent was removed under reduced pressure. The residue was purified by silica gel column chromatography using hexane as eluent to give the titled compound (3a) as a colorless oil in 90\% NMR yield determined by ${ }^{1} \mathrm{H}$ NMR analysis (isolated yield: $53 \%, 52.9 \mathrm{mg}$ ).

$17 \%$ isomer (structure in parentheses) is including in crude mixture determined by ${ }^{1} \mathrm{H}$ NMR analysis. ${ }^{1} \mathrm{H}$ and ${ }^{13} \mathrm{C}$ NMR were in agreement with the reference ${ }^{1}{ }^{1} \mathbf{H}$ NMR $\left(\mathbf{C D C l}_{3}, \mathbf{5 0 0} \mathbf{M H z}\right): 5.99-5.80(\mathrm{~m}, 2 \mathrm{H}), 2.15-2.10(\mathrm{~m}, 2 \mathrm{H})$, 1.53-1.45 (m, 6H), 1.33-1.26 (m, 13H) 0.92-0.85 (m, 19H). ${ }^{13} \mathbf{C}$ NMR (CDCl 3 , 125 MHz): 150.0, 127.1, 38.1, $31.9,30.1,29.4,28.9,27.4,22.8,17.7,13.9,9.5 . \mathbf{R}_{\mathbf{f}}=0.91$ (hexane). EI-MS: $m / z:[\mathrm{M}-\mathrm{Bu}]^{+}$calcd for $\mathrm{C}_{16} \mathrm{H}_{33} \mathrm{Sn}$ 345.2; Found 345.1.

Isomer: ${ }^{1} \mathbf{H}$ NMR $\left(\mathbf{C D C l}_{3}, \mathbf{5 0 0} \mathbf{~ M H z}\right)$ : 6.55-6.48 (m, 1H), 5.79-5.75 (m, 1H), 2.04-1.99 (m, 2H), 1.53-1.45 (m, 6H), 1.33-1.26 (m, 13H) 0.92-0.85 (m, 19H). ${ }^{13} \mathbf{C}$ NMR ( $\mathbf{C D C l}_{3}, \mathbf{1 2 5} \mathbf{~ M H z ) : ~ 1 4 9 . 5 , ~ 1 2 7 . 8 , ~ 3 7 . 3 , ~ 3 2 . 0 , ~ 3 0 . 1 , ~ 2 9 . 3 , ~}$ $29.0,27.5,22.8,17.7,14.2,10.4$.

(E)-tributyl(4-phenylbut-1-en-1-yl)stannane (3b)

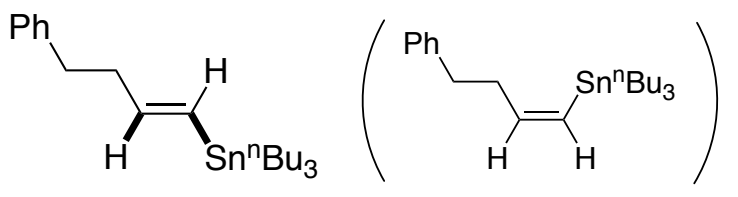

Using the Typical Procedure A (solvent: dioxane $1 \mathrm{~mL}$ ), the titled compound was obtained as a colorless oil in $89 \%$ NMR yield determined by ${ }^{1} \mathrm{H}$ NMR analysis (isolated yield: 66\%, 70.0 $\mathrm{mg}$ ) from 4-phenyl-1-butyne (1b, $32.5 \mathrm{mg}, 0.25 \mathrm{mmol})$.

$16 \%$ isomer (structure in parentheses) is including in crude mixture determined by ${ }^{1} \mathrm{H}$ NMR analysis. ${ }^{1} \mathbf{H}$ NMR $\left(\mathbf{C D C l}_{3}, \mathbf{5 0 0} \mathbf{M H z}\right):$ 7.31-7.24 (m, 2H), 7.22-7.14 (m, 3H), 6.06-5.81 (m, 2H), 2.75-2.66 (m, 2H), 2.48-2.42 (m, 2H), 1.52-1.44 (m, 6H), 1.30 (tq, 6H, $J=7.4,7.2 \mathrm{~Hz}), 0.92-0.83(\mathrm{~m}, 15 \mathrm{H}) .{ }^{13} \mathbf{C ~ N M R}\left(\mathbf{C D C l}_{3}, \mathbf{1 2 5} \mathbf{~ M H z}\right): 148.2$, $142.3,128.6,128.5,128.3,125.8,39.7,35.6,29.2,27.4,13.8,9.5 . \mathbf{R}_{\mathbf{f}}=0.85$ (hexane). $\mathbf{H R M S}(\mathbf{E S I}): \mathrm{m} / \mathrm{z}:[\mathrm{M}+\mathrm{Na}]^{+}$ calcd for $\mathrm{C}_{22} \mathrm{H}_{38} \mathrm{SnNa} 445.1893$; Found 445.1881. ATR-FTIR (neat) v: 2955, 2923, 2852, 1598, 1455, 1338, 1074, $988,696 \mathrm{~cm}^{-1}$.

Isomer: ${ }^{1} \mathbf{H}$ NMR $\left(\mathbf{C D C l}_{3}, \mathbf{5 0 0} \mathbf{~ M H z}\right)$ : 7.31-7.24 (m, 2H), 7.22-7.14 (m, 3H), 6.60-6.52 (m, 1H), 6.06-5.81 (m, $1 \mathrm{H}), 2.75-2.66(\mathrm{~m}, 2 \mathrm{H}), 2.37-2.31(\mathrm{~m}, 2 \mathrm{H}), 1.52-1.44(\mathrm{~m}, 6 \mathrm{H}), 1.30(\mathrm{tq}, 6 \mathrm{H}, J=7.4,7.2 \mathrm{~Hz}), 0.92-0.83(\mathrm{~m}, 15 \mathrm{H})$.

${ }^{13} \mathbf{C}$ NMR ( $\mathbf{C D C l}_{3}, \mathbf{1 2 5}$ MHz): 148.6, 142.0, 128.9, 128.6, 128.2, 126.0, 39.1, 36.4, 29.3 , 27.4, 14.2, 10.4. 
(E)-tributyl(2-cyclopropylvinyl)stannane (3c)

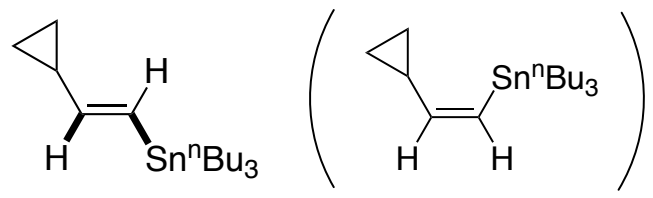

Using the Typical Procedure A (solvent: dioxane $1 \mathrm{~mL}$, purified by gel permeation chromatography instead of silica gel column chromatography), the titled compound was obtained as a colorless oil in $77 \%$ yield NMR yield determined by ${ }^{1} \mathrm{H}$ NMR analysis (isolated yield: $31 \%, 27.3 \mathrm{mg}$ ) from cyclopropylacetylene (1c, $16.5 \mathrm{mg}, 0.25 \mathrm{mmol})$.

$11 \%$ isomer (structure in parentheses) is including in crude mixture determined by ${ }^{1} \mathrm{H}$ NMR analysis. ${ }^{1} \mathrm{H}$ and ${ }^{13} \mathrm{C}$ NMR were in agreement with the reference ${ }^{2}{ }^{1} \mathbf{H}$ NMR $\left(\mathbf{C D C l}_{3}, \mathbf{5 0 0} \mathbf{~ M H z}\right): 6.04-5.87(\mathrm{~m}, 1 \mathrm{H}), 5.53-5.34(\mathrm{~m}, 1 \mathrm{H})$, 1.55-1.42 (m, 7H), 1.33-1.27 (m, 6H), 0.94-0.82 (m, 15H), 0.75-0.68 (m, 2H), 0.44-0.39 (m, 2H). ${ }^{13} \mathbf{C}$ NMR $\left(\mathbf{C D C l}_{3}, \mathbf{1 2 5} \mathbf{M H z}\right): 152.9,124.0,29.2,27.4,18.3,13.9,9.6,7.2 . \mathbf{R}_{\mathrm{f}}=0.90$ (hexane). EI-MS: $\mathrm{m} / z:[\mathrm{M}-\mathrm{Bu}]^{+} \mathrm{calcd}$ for $\mathrm{C}_{13} \mathrm{H}_{25} \mathrm{Sn} 301.1$; Found 301.1 .

Isomer: ${ }^{1} \mathbf{H}$ NMR $\left(\mathbf{C D C l}_{3}, \mathbf{5 0 0} \mathbf{~ M H z}\right):$ 5.87-5.81 (m, 1H), 5.69-5.62 (m, 1H), 1.55-1.42 (m, 7H), 1.33-1.27 (m, 6H), 0.94-0.82 (m, 15H), 0.75-0.68 (m, 2H), 0.44-0.39 (m, 2H). ${ }^{13} \mathbf{C}$ NMR (CDCl 3 , 125 MHz): 152.9, 124.5, 29.4, $27.6,17.5,13.9,8.9,7.5$.

(E)-tributyl(2-cyclopentylvinyl)stannane (3d)

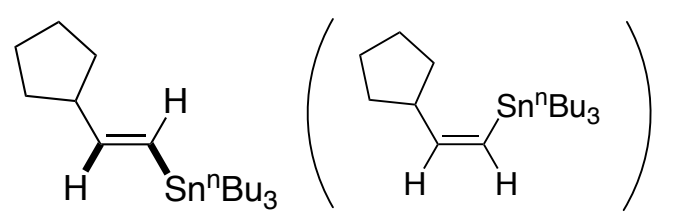

Using the Typical Procedure A (solvent: dioxane $1 \mathrm{~mL}$ ), the titled compound was obtained as a colorless oil in $84 \%$ NMR yield determined by ${ }^{1} \mathrm{H}$ NMR analysis (isolated yield: $58 \%, 55.7 \mathrm{mg}$ ) from cyclopentylacetylene (1d, $23.5 \mathrm{mg}, 0.25 \mathrm{mmol}$ ).

$19 \%$ isomer (structure in parentheses) is including in crude mixture determined by ${ }^{1} \mathrm{H}$ NMR analysis. ${ }^{1} \mathbf{H}$ NMR $\left(\mathbf{C D C l}_{\mathbf{3}}, \mathbf{5 0 0} \mathbf{~ M H z}\right)$ : $5.98-5.80$ (m, 2H), 2.50-2.43 (m, 1H), 1.81-1.71 $(\mathrm{m}, 2 \mathrm{H}), 1.69-1.60(\mathrm{~m}, 2 \mathrm{H}), 1.58-1.46(\mathrm{~m}, 8 \mathrm{H}), 1.37-1.26(\mathrm{~m}, 8 \mathrm{H}), 0.94-0.82(\mathrm{~m}, 15 \mathrm{H}) .{ }^{13} \mathbf{C} \mathbf{~ N M R}\left(\mathbf{C D C l}_{3}, \mathbf{1 2 5}\right.$ MHz): $154.4,124.4,48.1,32.8,29.3,27.4,25.4,13.9,9.5 . \mathbf{R}_{\mathbf{f}}=0.87$ (hexane). HRMS (APCI): $m / z:[\mathrm{M}-\mathrm{Bu}]^{+}$ calcd for $\mathrm{C}_{15} \mathrm{H}_{29} \mathrm{Sn} 329.1286$; Found 329.1257. ATR-FTIR (neat) v: 2953, 2925, 2871, 1596, 1456, 1376, 1070, $987,870,689,665 \mathrm{~cm}^{-1}$.

Isomer: ${ }^{1} \mathbf{H}$ NMR $\left(\mathbf{C D C l}_{3}, \mathbf{5 0 0} \mathbf{M H z}\right)$ : 6.42-6.36 (m, 1H), 5.70-5.65 (m, 1H), 2.24-2.17 (m, 1H), 1.81-1.71 (m, $2 \mathrm{H}), 1.69-1.60(\mathrm{~m}, 2 \mathrm{H}), 1.58-1.46(\mathrm{~m}, 8 \mathrm{H}), 1.37-1.26(\mathrm{~m}, 8 \mathrm{H}), 0.94-0.82(\mathrm{~m}, 15 \mathrm{H}) .{ }^{13} \mathbf{C} \mathbf{~ N M R}\left(\mathbf{C D C l}_{3}, \mathbf{1 2 5} \mathbf{M H z}\right)$ : $154.2,125.7,48.5,33.9,29.4,27.5,25.8,13.9,10.5$.

(E)-tributyl(5-chloropent-1-en-1-yl)stannane (3e)

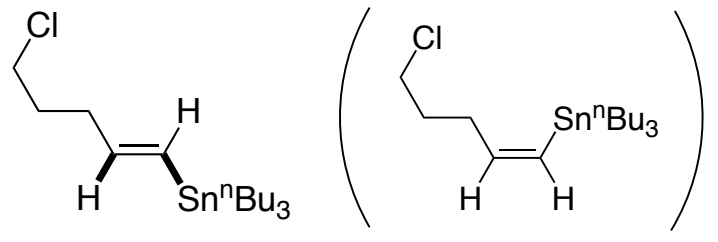

Using the Typical Procedure A (fluoride source: KF + 18-crown6 instead of CsF, solvent: dioxane $1 \mathrm{~mL}$ ), the titled compound was obtained as a colorless oil in $97 \%$ NMR yield determined by ${ }^{1} \mathrm{H}$ NMR analysis (isolated yield: $59 \%, 57.6 \mathrm{mg}$ ) from 5-chloro-1pentyne (1e, $25.6 \mathrm{mg}, 0.25 \mathrm{mmol}$ ).

$13 \%$ isomer (structure in parentheses) is including in crude mixture determined by ${ }^{1} \mathrm{H}$ NMR analysis. ${ }^{1} \mathbf{H}$ NMR $\left(\mathbf{C D C l}_{3}, \mathbf{5 0 0} \mathbf{M H z}\right): 5.99-5.85(\mathrm{~m}, 2 \mathrm{H}), 3.53(\mathrm{t}, 2 \mathrm{H}, J=6.8 \mathrm{~Hz}), 2.31-2.25(\mathrm{~m}, 2 \mathrm{H}), 1.88(\mathrm{tt}, 2 \mathrm{H}, J=7.2,7.2 \mathrm{~Hz})$, 1.53-1.43 (m, 6H), 1.30 (tq, 6H, $J=7.4,7.2 \mathrm{~Hz}), 0.94-0.84(\mathrm{~m}, 15 \mathrm{H}) \cdot{ }^{13} \mathbf{C} \mathbf{N M R}\left(\mathbf{C D C l}_{3}, \mathbf{1 2 5} \mathbf{~ M H z}\right): 147.1,129.3$, 44.4, 34.8, 31.7, 29.1, 27.2, 13.7, 9.4. $\mathrm{R}_{\mathrm{f}}=0.90$ (hexane). HRMS (APCI): $m / z:[\mathrm{M}-\mathrm{Bu}]^{+}$calcd for $\mathrm{C}_{13} \mathrm{H}_{26} \mathrm{ClSn}$ 337.0740; Found 337.0704. ATR-FTIR (neat) v: 2955, 2924, 2871, 2845, 1599, 1458, 989, 865, 689, $656 \mathrm{~cm}^{-1}$.

Isomer: ${ }^{1} \mathbf{H}$ NMR $\left(\mathbf{C D C l}_{3}, \mathbf{5 0 0} \mathbf{~ M H z}\right)$ : 6.51-6.44 (m, 1H), 5.99-5.85 (m, 1H), 3.56-3.51 (m, 2H), 2.31-2.25 (m, $2 \mathrm{H}), 1.88(\mathrm{tt}, 2 \mathrm{H}, J=7.2,7.2 \mathrm{~Hz}), 1.53-1.43(\mathrm{~m}, 6 \mathrm{H}), 1.30(\mathrm{tq}, 6 \mathrm{H}, J=7.4,7.2 \mathrm{~Hz}), 0.94-0.84(\mathrm{~m}, 15 \mathrm{H}) .{ }^{13} \mathbf{C} \mathbf{N M R}$ $\left(\mathbf{C D C l}_{3}, \mathbf{1 2 5} \mathbf{M H z}\right):$ 146.9, 129.9, 44.5, 34.1, 32.7, 29.2, 27.3, 13.7, 10.2. 
(E)-tributyl(3-methoxyprop-1-en-1-yl)stannane (3f)<smiles>CCCCCCCCCCCCCCCCCCC</smiles>

from methyl propargyl ether (1f, $17.5 \mathrm{mg}, 0.25 \mathrm{mmol}$ ).

$19 \%$ isomer (structure in parentheses) is including in crude mixture determined by ${ }^{1} \mathrm{H}$ NMR analysis. ${ }^{1} \mathbf{H}$ NMR $\left(\mathbf{C D C l}_{3}, \mathbf{5 0 0} \mathbf{M H z}\right): 6.31-5.95$ (m, 2H), 3.97-3.93 (m, 2H), 3.34 (s, 3H), 1.55-1.43 (m, 6H), 1.30 (tq, 6H, $J=7.4$, 7.4 Hz), 0.95-0.81 (m, 15H). ${ }^{13} \mathbf{C} \mathbf{~ N M R}\left(\mathbf{C D C l}_{3}, 125 \mathbf{~ M H z}\right): 144.6,131.4,58.0,29.2,27.4,13.8,10.9$ 9.6. $\mathbf{R}_{\mathbf{f}}=$ 0.23 (hexane:AcOEt = 40:1). HRMS (APCI): $\mathrm{m} / z$ : $[\mathrm{M}-\mathrm{Bu}]^{+}$calcd for $\mathrm{C}_{12} \mathrm{H}_{25} \mathrm{OSn}$ 305.0922; Found 305.0918. ATR-FTIR (neat) v: 2955, 2921, 2871, 1604, 1463, 1115, 996, 690, $660 \mathrm{~cm}^{-1}$.

Isomer: ${ }^{1} \mathbf{H}$ NMR $\left(\mathbf{C D C l}_{3}, \mathbf{5 0 0} \mathbf{M H z}\right)$ : 6.66-6.57 (m, 1H), 6.31-5.95 (m, 1H), 3.92-3.88 (m, 2H), $3.33(\mathrm{~s}, 3 \mathrm{H})$, 1.55-1.43 (m, 6H), 1.30 (tq, 6H, $J=7.4,7.4 \mathrm{~Hz}), 0.95-0.81(\mathrm{~m}, 15 \mathrm{H}) .{ }^{13} \mathbf{C} \mathbf{N M R}\left(\mathbf{C D C l}_{3}, \mathbf{1 2 5} \mathbf{M H z}\right): 144.1,132.0$, $58.2,29.3,27.5,13.8,10.9,9.7$.

\section{(E)-7-(tributylstannyl)hept-6-enenitrile (3g)}

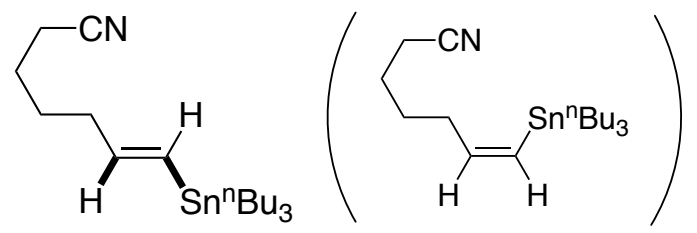

Using the Typical Procedure A (fluoride source: KF + 18-crown6 instead of $\mathrm{CsF}$, solvent: dioxane $1 \mathrm{~mL}$ ), the titled compound was obtained as a colorless oil in $>99 \%$ NMR yield determined by ${ }^{1} \mathrm{H}$ NMR analysis (isolated yield: $54 \%, 54.2 \mathrm{mg}$ ) from 6-heptynenitrile (1g, $26.8 \mathrm{mg}, 0.25 \mathrm{mmol})$.

$29 \%$ isomer (structure in parentheses) is including in crude mixture determined by ${ }^{1} \mathrm{H}$ NMR analysis. ${ }^{1} \mathbf{H}$ NMR $\left(\mathbf{C D C l}_{3}, \mathbf{5 0 0} \mathbf{M H z}\right): 6.01-5.75(\mathrm{~m}, 2 \mathrm{H}), 2.34(\mathrm{t}, 2 \mathrm{H}, J=7.1 \mathrm{~Hz}), 2.21-2.14(\mathrm{~m}, 2 \mathrm{H}), 1.71-1.64(\mathrm{~m}, 2 \mathrm{H}), 1.61-1.53$ $(\mathrm{m}, 2 \mathrm{H}), 1.53-1.45(\mathrm{~m}, 6 \mathrm{H}), 1.30(\mathrm{tq}, 6 \mathrm{H}, J=7.5,7.2 \mathrm{~Hz}), 0.93-0.85(\mathrm{~m}, 15 \mathrm{H}) .{ }^{13} \mathbf{C} \mathbf{~ N M R}\left(\mathbf{C D C l}_{3}, 125 \mathrm{MHz}\right)$ : 148.0, 129.0, 119.9, 36.9, 29.3, 27.9, 27.4, 24.9, 17.2, 13.9, 9.6. $\mathbf{R}_{\mathbf{f}}=0.23$ (hexane:AcOEt = 20:1). HRMS (APCI): $m / z:[\mathrm{M}+\mathrm{H}]^{+}$calcd for $\mathrm{C}_{19} \mathrm{H}_{37} \mathrm{NSn} 400.2021$; Found 400.2023 . ATR-FTIR (neat) v: 2954, 2925, 1599, 1462, 1071, $989,865,690,661 \mathrm{~cm}^{-1}$.

Isomer: ${ }^{1} \mathbf{H}$ NMR $\left(\mathbf{C D C l}_{3}, \mathbf{5 0 0} \mathbf{M H z}\right): 6.51-6.43(\mathrm{~m}, 1 \mathrm{H}), 6.01-5.75(\mathrm{~m}, 1 \mathrm{H}), 2.34(\mathrm{t}, 2 \mathrm{H}, J=7.1 \mathrm{~Hz}), 2.10-2.04$ $(\mathrm{m}, 2 \mathrm{H}), 1.71-1.64(\mathrm{~m}, 2 \mathrm{H}), 1.61-1.53(\mathrm{~m}, 2 \mathrm{H}), 1.53-1.45(\mathrm{~m}, 6 \mathrm{H}), 1.30$ (tq, 6H, $J=7.5,7.2 \mathrm{~Hz}), 0.93-0.85$ (m, 15H). ${ }^{13} \mathbf{C} \mathbf{~ N M R}\left(\mathbf{C D C l}_{3}, \mathbf{1 2 5} \mathbf{M H z}\right): 147.7,129.5,119.7,36.1,29.3,28.9,27.5,25.2,17.3,13.9,10.4$.

(E)-6-(tributylstannyl)hex-5-en-1-ol (3h)

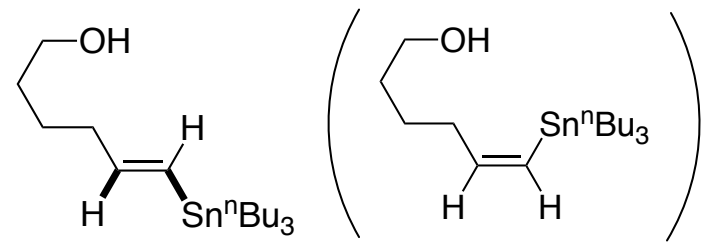

Using the Typical Procedure A (solvent: dioxane $1 \mathrm{~mL}$, purified by gel permeation chromatography instead of silica gel column chromatography) the titled compound was obtained as a colorless oil in $>99 \%$ NMR yield determined by ${ }^{1} \mathrm{H}$ NMR analysis (isolated yield: $67 \%, 65.4 \mathrm{mg})$ from hex-5-yn-1-ol $(\mathbf{1 h}, 24.5 \mathrm{mg}, 0.25$

mmol).

$16 \%$ isomer (structure in parentheses) is including in crude mixture determined by ${ }^{1} \mathrm{H}$ NMR analysis. ${ }^{1} \mathrm{H}$ and ${ }^{13} \mathrm{C}$ NMR were in agreement with the reference ${ }^{3} .{ }^{1} \mathbf{H}$ NMR $\left(\mathbf{C D C l}_{3}, \mathbf{5 0 0} \mathbf{~ M H z}\right): 6.02-5.77(\mathrm{~m}, 2 \mathrm{H}), 3.69$ (bs, $\left.1 \mathrm{H}\right)$, 3.68-3.63 (m, 2H), 2.20-2.14 (m, 2H), 1.62-1.56 (m, 2H), 1.53-1.44 (m, 8H), 1.30 (tq, 6H, J = 7.5, 7.1 Hz), 0.92$0.83(\mathrm{~m}, 15 \mathrm{H}) .{ }^{13} \mathbf{C} \mathbf{~ N M R}\left(\mathbf{C D C l}_{\mathbf{3}}, \mathbf{1 2 5} \mathbf{M H z}\right): 149.3,127.8,63.1,37.7,32.4,29.3,27.4,25.2,13.9,9.5 . \mathbf{R}_{\mathbf{f}}=0.28$ (hexane:AcOEt = 20:1). EI-MS: $m / z:[\mathrm{M}-\mathrm{Bu}]^{+}$calcd for $\mathrm{C}_{17} \mathrm{H}_{27} \mathrm{OSn} 333.1$; Found 333.1.

Isomer: ${ }^{1} \mathrm{H}$ and ${ }^{13} \mathrm{C}$ NMR were in agreement with the reference ${ }^{4}{ }^{1} \mathbf{H} \mathbf{~ N M R}\left(\mathbf{C D C l}_{3}, \mathbf{5 0 0} \mathbf{~ M H z}\right): 6.54-6.47(\mathrm{~m}, 1 \mathrm{H})$, 6.02-5.77 (m, 1H), $3.69(\mathrm{bs}, 1 \mathrm{H}), 3.68-3.63(\mathrm{~m}, 2 \mathrm{H}), 2.09-2.03(\mathrm{~m}, 2 \mathrm{H}), 1.62-1.56(\mathrm{~m}, 2 \mathrm{H}), 1.53-1.44(\mathrm{~m}, 8 \mathrm{H})$, 
1.31 (tq, $6 \mathrm{H}, J=7.3,7.0 \mathrm{~Hz}), 0.92-0.83(\mathrm{~m}, 15 \mathrm{H}) .{ }^{13} \mathbf{C} \mathbf{N M R}\left(\mathbf{C D C l}_{3}, \mathbf{1 2 5} \mathbf{M H z}\right): 148.8,128.5,63.1,36.9,32.7$, $29.4,27.5,25.2,13.9,10.4$.

\section{(E)-tributyl(dec-1-en-5-yn-1-yl)stannane (3i)}

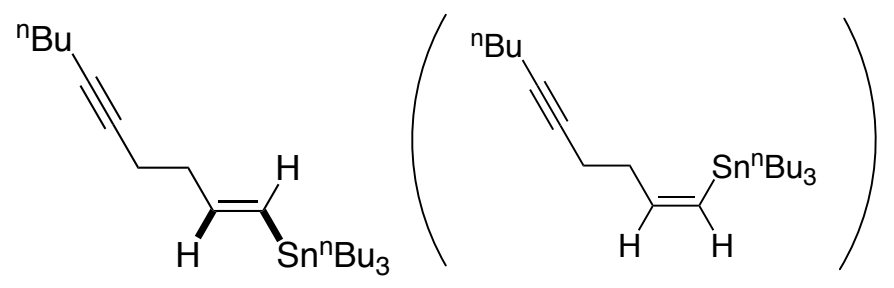

Using the Typical Procedure A (fluoride source: KF + 18-crown-6 instead of CsF, solvent: dioxane $1 \mathrm{~mL}$ ), the titled compound was obtained as a colorless oil in $56 \%$ NMR yield determined by ${ }^{1} \mathrm{H}$ NMR analysis (isolated yield: $42 \%, 45.2 \mathrm{mg}$ ) from 1,5-decadiyne (1i, $33.6 \mathrm{mg}, 0.25 \mathrm{mmol})$.

$14 \%$ isomer (structure in parentheses) is including in crude mixture determined by ${ }^{1} \mathrm{H}$ NMR analysis. ${ }^{1} \mathbf{H}$ NMR ( $\left.\mathbf{C D C l}_{3}, \mathbf{5 0 0} \mathbf{M H z}\right)$ : 6.06-5.84 (m, 2H), 2.34-2.27 (m, 2H), 2.26-2.19 (m, 2H), 2.18-2.11 (m, 2H), 1.43-1.42 (m, 8H), 1.34-1.28 (m, 6H), 0.95-0.82 (m, 15H). ${ }^{13} \mathbf{C} \mathbf{~ N M R}\left(\mathbf{C D C l}_{3}, \mathbf{1 2 5} \mathbf{~ M H z}\right):$ 147.7, 128.6, 80.7, 79.8, 37.3, 31.4, 31.1, 29.2, 27.4, 22.1 , 18.6, 13.8, 10.4, 9.5. $\mathbf{R}_{\mathbf{f}}=0.69$ (hexane). HRMS (APCI): $m / z:[\mathrm{M}-\mathrm{Bu}]^{+}$calcd for $\mathrm{C}_{18} \mathrm{H}_{33} \mathrm{Sn}$ 369.1599; Found 369.1571. ATR-FTIR (neat) v: 2955, 2924, 2854, 1599, 1463, 1377, 1250, 1070, 841, $668 \mathrm{~cm}^{-}$ 1 .

Isomer: ${ }^{1} \mathbf{H}$ NMR $\left(\mathbf{C D C l}_{3}, \mathbf{5 0 0} \mathbf{M H z}\right)$ : 6.59-6.51 (m, 1H), 6.06-5.84 (m, 1H), 2.34-2.27 (m, 2H), 2.26-2.19 (m, 2H), 2.18-2.11 (m, 2H), 1.43-1.42 (m, 8H), 1.34-1.28 (m, 6H), 0.95-0.82 (m, 15H). ${ }^{13} \mathbf{C}$ NMR (CDCl 3 , 125 MHz): $147.7,128.6,80.7,79.8,37.3,31.3,31.1,29.3,27.4,22.1,18.8,13.8,10.4,9.5$.

(8R,9S,13S,14S,17R)-3-methoxy-13-methyl-17-((E)-2-(tributylstannyl)vinyl)-7,8,9,11,12,13,14,15,16,17decahydro-6H-cyclopenta[a]phenanthren-17-ol (3j)

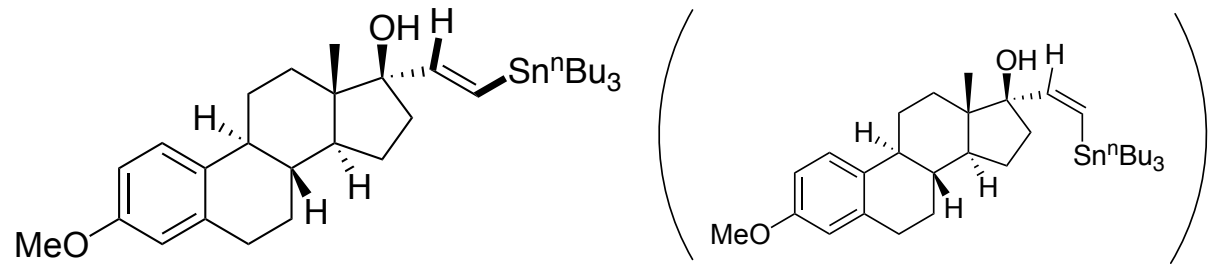

Using the Typical Procedure A (fluoride source: $\mathrm{KF}+18$ crown-6 instead of $\mathrm{CsF}$ ), the titled compound was obtained as a colorless oil in $>99 \%$ NMR yield determined by ${ }^{1} \mathrm{H}$ NMR

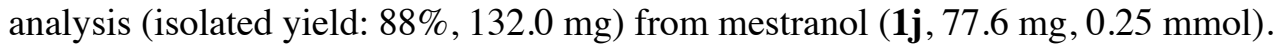

$28 \%$ isomer (structure in parentheses) is including in crude mixture determined by ${ }^{1} \mathrm{H}$ NMR analysis. ${ }^{1} \mathbf{H}$ NMR $\left(\mathbf{5 0 0} \mathbf{~ M H z}, \mathbf{C D C l}_{3}\right): 7.19(\mathrm{~d}, 1 \mathrm{H}, J=8.6 \mathrm{~Hz}), 6.70(\mathrm{dd}, 1 \mathrm{H}, J=8.5,2.7 \mathrm{~Hz}), 6.63(\mathrm{~d}, 1 \mathrm{H}, J=2.6 \mathrm{~Hz}), 6.30-5.96$ $(\mathrm{m}, 2 \mathrm{H}), 3.77(\mathrm{~s}, 3 \mathrm{H}), 2.90-2.81(\mathrm{~m}, 2 \mathrm{H}), 2.31-2.24(\mathrm{~m}, 1 \mathrm{H}), 2.12-2.01(\mathrm{~m}, 2 \mathrm{H}), 1.93-1.82(\mathrm{~m}, 2 \mathrm{H}), 1.77-1.69(\mathrm{~m}$, 1H), 1.61-1.57 (m, 2H), 1.54-1.39 (m, 11H), 1.36-1.28 (m, 7H), 0.96-0.86 (m, 18H) $\left.{ }^{13} \mathbf{C ~ N M R ~ ( 1 2 5 ~ M H z , ~ C D C l ~}\right)$ : 157.6, 152.6, 138.1 132.8, 126.5, 124.7, 113.9, 111.6, 85.6, 55.3, 49.2, 46.8, 44.0, 39.6, 36.1, 32.5, 30.1, 29.3, 27.6, 27.4 26.5 23.6, 14.4, 13.9, 9.8. $\mathbf{R}_{\mathbf{f}}=0.32$ (hexane:AcOEt = 10:1). HRMS (APCI): $\mathrm{m} / z:[\mathrm{M}-\mathrm{Bu}]^{+}$calcd for $\mathrm{C}_{29} \mathrm{H}_{45} \mathrm{O}_{2} \mathrm{Sn}$ 545.2436; Found 545.2439. ATR-FTIR (neat) v: 2950, 2925, 2870, 1609, 1498, 1377, 1254, 1153, $997,685 \mathrm{~cm}^{-1}$.

\section{(E)-tributyl(styryl)stannane (3k)}

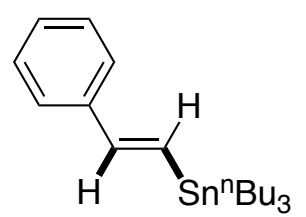

Using the Typical Procedure A (fluoride source: KF + 18-crown-6 instead of CsF, proton source: $\mathrm{H}_{2} \mathrm{O}$ instead of $\mathrm{MeOH}$, solvent: dioxane $1 \mathrm{~mL}$ ), the titled compound was obtained as a colorless oil in $80 \%$ NMR yield determined by ${ }^{1} \mathrm{H}$ NMR analysis (isolated yield: $56 \%, 55.0$ $\mathrm{mg}$ ) from ethynylbenzene (1k, $25.5 \mathrm{mg}, 0.25 \mathrm{mmol})$.

${ }^{1} \mathrm{H}$ and ${ }^{13} \mathrm{C}$ NMR were in agreement with the reference ${ }^{3} .{ }^{1} \mathbf{H}$ NMR $\left(500 \mathbf{~ M H z}, \mathbf{C D C l}_{3}\right): 7.41$ 
$(\mathrm{d}, 2 \mathrm{H}, J=7.2 \mathrm{~Hz}), 7.32(\mathrm{dd}, 2 \mathrm{H}, J=7.0,7.0 \mathrm{~Hz}), 7.22(\mathrm{tt}, 1 \mathrm{H}, J=7.3,1.1 \mathrm{~Hz}), 6.93-6.80(\mathrm{~m}, 2 \mathrm{H}), 1.57-1.51(\mathrm{~m}$, $6 \mathrm{H}), 1.33$ (tq, $6 \mathrm{H}, J=7.5,7.2 \mathrm{~Hz}), 1.04-0.92(\mathrm{~m}, 6 \mathrm{H}), 0.90$ (t, 9H, $J=7.3 \mathrm{~Hz}) .{ }^{13} \mathbf{C ~ N M R}\left(\mathbf{1 2 5} \mathbf{M H z}, \mathbf{C D C l}_{3}\right)$ : $146.0,138.8,129.6,128.5,127.5,126.0,29.1,27.3,13.7,9.6 . \mathbf{R}_{\mathbf{f}}=0.57$ (hexane). EI-MS: $m / z:[\mathrm{M}-\mathrm{Bu}]^{+}$calcd for $\mathrm{C}_{16} \mathrm{H}_{25} \mathrm{Sn} 337.1$; Found 337.1.

\section{(E)-tributyl(4-chlorostyryl)stannane (3l)}

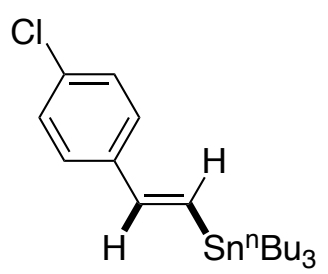

Using the Typical Procedure A (fluoride source: KF + 18-crown-6 instead of CsF, proton source: $\mathrm{H}_{2} \mathrm{O}$ instead of $\mathrm{MeOH}$, (solvent: dioxane $1 \mathrm{~mL}$ ), the titled compound was obtained as a colorless oil in 79\% NMR yield determined by ${ }^{1} \mathrm{H}$ NMR analysis (isolated yield: $76 \%$, $81.5 \mathrm{mg}$ ) from 1-chloro-4-ethynylbenzene (11, $34.1 \mathrm{mg}, 0.25 \mathrm{mmol})$.

${ }^{1} \mathbf{H} \mathbf{N M R}\left(\mathbf{C D C l}_{3}, \mathbf{5 0 0} \mathbf{M H z}\right): 7.33(\mathrm{dd}, 2 \mathrm{H}, J=8.7,2.0 \mathrm{~Hz}), 7.28(\mathrm{dd}, 2 \mathrm{H}, J=8.6,1.9 \mathrm{~Hz})$, 6.90-6.75 (m, 2H), 1.58-1.50 (m, 6H), $1.33(\mathrm{tq}, 6 \mathrm{H}, J=7.4,7.2 \mathrm{~Hz}), 1.01-0.93(\mathrm{~m}, 6 \mathrm{H})$, $0.90(\mathrm{t}, 9 \mathrm{H}, J=7.3 \mathrm{~Hz}) .{ }^{13} \mathbf{C} \mathbf{~ N M R}\left(\mathbf{C D C l}_{3}, \mathbf{1 2 5} \mathbf{~ M H z}\right): 144.6,137.3,133.1,130.7,128.6,127.2,29.1,27.3,13.7$, 9.6. $\mathbf{R}_{\mathbf{f}}=0.73$ (hexane). HRMS (APCI): $m / z:[\mathrm{M}-\mathrm{Bu}]^{+}$calcd for $\mathrm{C}_{16} \mathrm{H}_{24} \mathrm{ClSn}$ 371.0538; Found 371.0535. ATRFTIR (neat) v: 2955, 2924, 2852, 1591, 1488, 1182, 1092, 984, 841, $626 \mathrm{~cm}^{-1}$.

\section{(E)-tributyl(3-chlorostyryl)stannane (3m)}

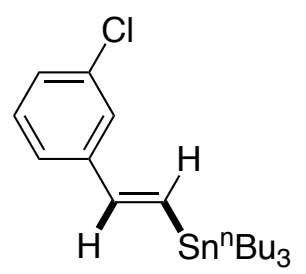

Using the Typical Procedure A (fluoride source: KF + 18-crown-6 instead of CsF, proton source: $\mathrm{H}_{2} \mathrm{O}$ instead of $\mathrm{MeOH}$, solvent: dioxane $1 \mathrm{~mL}$ ) the titled compound was obtained as a colorless oil in 98\% NMR yield determined by ${ }^{1} \mathrm{H}$ NMR analysis (isolated yield: 69\%, 74.3 $\mathrm{mg}$ ) from 1-chloro-3-ethynylbenzene (1m, $34.1 \mathrm{mg}, 0.25 \mathrm{mmol})$.

${ }^{1} \mathbf{H}$ NMR $\left(\mathbf{C D C l}_{3}, \mathbf{5 0 0} \mathbf{M H z}\right): 7.39(\mathrm{dd}, 1 \mathrm{H}, J=1.8,1.5 \mathrm{~Hz}), 7.28-7.17(\mathrm{~m}, 3 \mathrm{H}), 6.95-6.75$ $(\mathrm{m}, 2 \mathrm{H}), 1.59-1.50(\mathrm{~m}, 6 \mathrm{H}), 1.34(\mathrm{tq}, 6 \mathrm{H}, J=7.5,7.4 \mathrm{~Hz}), 1.02-0.93(\mathrm{~m}, 6 \mathrm{H}), 0.90(\mathrm{t}, 9 \mathrm{H}, J$ $=7.4 \mathrm{~Hz}) .{ }^{13} \mathbf{C} \mathbf{N M R}\left(\mathbf{C D C l}_{3}, \mathbf{1 2 5} \mathbf{~ M H z}\right): 144.6,140.8,134.7,132.1,129.8,127.5,126.1,124.4,29.2,27.4,13.8$, 9.8. $\mathbf{R}_{\mathbf{f}}=0.65$ (hexane). HRMS (APCI): $\mathrm{m} / z$ : $[\mathrm{M}-\mathrm{Bu}]^{+}$calcd for $\mathrm{C}_{16} \mathrm{H}_{24} \mathrm{ClSn} 371.0538$; Found 371.0579. ATRFTIR (neat) v: 2955, 2924, 2849, 1592, 1464, 1074, 984, 864, 756, $696 \mathrm{~cm}^{-1}$.

\section{(E)-tributyl(2-chlorostyryl)stannane (3n)}

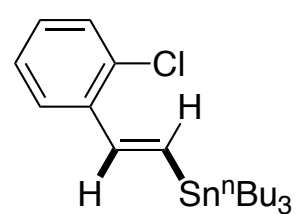

Using the Typical Procedure A (fluoride source: KF + 18-crown-6 instead of CsF, proton source: $\mathrm{H}_{2} \mathrm{O}$ instead of $\mathrm{MeOH}$, (solvent: dioxane $1 \mathrm{~mL}$ ), the titled compound was obtained as a colorless oil in 78\% NMR yield determined by ${ }^{1} \mathrm{H}$ NMR analysis (isolated yield: $73 \%$, $78.5 \mathrm{mg}$ ) from 1-chloro-2-ethynylbenzene (1n, $34.1 \mathrm{mg}, 0.25 \mathrm{mmol})$.

${ }^{1} \mathbf{H}$ NMR $\left(\mathbf{C D C l}_{3}, \mathbf{5 0 0} \mathbf{M H z}\right): 7.57(\mathrm{dd}, 1 \mathrm{H}, J=9.0,1.5 \mathrm{~Hz}), 7.33(\mathrm{dd}, 1 \mathrm{H}, J=9.0,1.5 \mathrm{~Hz})$, 7.29-7.21 (m, 2H), $7.15(\mathrm{td}, 1 \mathrm{H}, J=8.0,1.5 \mathrm{~Hz}), 6.95-6.75(\mathrm{~m}, 2 \mathrm{H}), 1.58-1.50(\mathrm{~m}, 6 \mathrm{H}), 1.34(\mathrm{tq}, 6 \mathrm{H}, J=7.4,7.2$ $\mathrm{Hz}), 1.01-0.93(\mathrm{~m}, 6 \mathrm{H}), 0.90(\mathrm{t}, 9 \mathrm{H}, J=7.3 \mathrm{~Hz}) .{ }^{13} \mathbf{C} \mathbf{N M R}\left(\mathbf{C D C l}_{3}, \mathbf{1 2 5} \mathbf{M H z}\right): 142.1,137.0,133.8,132.8,129.7$, $128.5,126.9,126.7,29.3,27.4,13.9,9.9 . \mathbf{R}_{\mathbf{f}}=0.71$ (hexane). HRMS (APCI): $m / z:[\mathrm{M}-\mathrm{Bu}]^{+}$calcd for $\mathrm{C}_{16} \mathrm{H}_{24} \mathrm{ClSn}$ 371.0538; Found 371.0572. ATR-FTIR (neat) v: 2955, 2924, 2850, 1583, 1463, 1050, 987, 865, 744, $656 \mathrm{~cm}^{-1}$.

\section{(E)-tributyl(4-(trifluoromethyl)styryl)stannane (3o)}

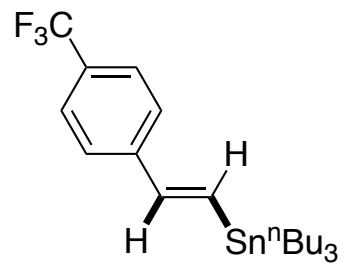

Using the Typical Procedure A (fluoride source: KF + 18-crown-6 instead of CsF, proton source: $\mathrm{H}_{2} \mathrm{O}$ instead of $\mathrm{MeOH}$, solvent: dioxane $1 \mathrm{~mL}$ ), the titled compound was obtained as a yellow solid in 64\% NMR yield determined by ${ }^{1} \mathrm{H}$ NMR analysis (isolated yield: $46 \%$, $131.6 \mathrm{mg}$ ) from 1-ethynyl-4-(trifluoromethyl)benzene (1o, $42.5 \mathrm{mg}, 0.25 \mathrm{mmol}) .60 \mathrm{wt} \%$ of hexa- $n$-butyl-distannane $\left({ }^{n} \mathrm{Bu}_{3} \mathrm{Sn}-\mathrm{Sn}^{\mathrm{n}} \mathrm{Bu}_{3}\right)$ is including in an isolated product.

${ }^{1} \mathbf{H}$ NMR $\left(\mathbf{C D C l}_{3}, \mathbf{5 0 0} \mathbf{M H z}\right): 7.56(\mathrm{~d}, 2 \mathrm{H}, J=8.3 \mathrm{~Hz}), 7.49$ (d, 2H, $\left.J=8.0 \mathrm{~Hz}\right), 7.06-$ 
$6.85(\mathrm{~m}, 2 \mathrm{H}), 1.58-1.50(\mathrm{~m}, 6 \mathrm{H}), 1.33(\mathrm{tq}, 6 \mathrm{H}, J=7.4,7.2 \mathrm{~Hz}), 1.01-0.93(\mathrm{~m}, 6 \mathrm{H}), 0.90(\mathrm{t}, 9 \mathrm{H}, J=7.3 \mathrm{~Hz}) .{ }^{13} \mathrm{C}$ NMR $\left(\mathbf{C D C l}_{3}, \mathbf{1 2 5} \mathbf{M H z}\right): 144.7,142.1,140.0,129.2(\mathrm{~J}=28.8 \mathrm{~Hz}), 126.2,125.6(\mathrm{~J}=282.5 \mathrm{~Hz}), 125.6(\mathrm{~J}=3.8$ $\mathrm{Hz}), 29.2,27.4,17.7,13.8,9.8 .{ }^{19} \mathbf{F} \mathbf{~ N M R}\left(\mathbf{C D C l}_{3}, 376 \mathbf{M H z}\right):-62.39 . \mathbf{R}_{\mathbf{f}}=0.68$ (hexane). HRMS (APCI): $\mathrm{m} / \mathrm{z}:$ $[\mathrm{M}-\mathrm{Bu}]^{+}$calcd for $\mathrm{C}_{17} \mathrm{H}_{24} \mathrm{~F}_{3} \mathrm{Sn}$ 405.0847; Found 405.0840. ATR-FTIR (neat) v: 2957, 2923, 2853, 1613, 1464, $1322,1124,1066,852,598 \mathrm{~cm}^{-1}$.

\section{(E)-(2-([1,1'-biphenyl]-4-yl)vinyl)tributylstannane (3p)}

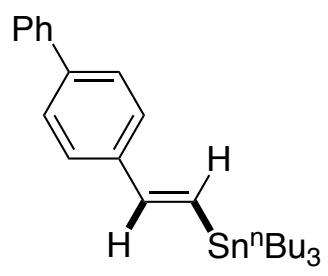

Using the Typical Procedure A (fluoride source: KF + 18-crown-6 instead of CsF, proton source: $\mathrm{H}_{2} \mathrm{O}$ instead of $\mathrm{MeOH}$, solvent: dioxane $1 \mathrm{~mL}$ ), the titled compound was obtained as a colorless oil in $83 \%$ NMR yield determined by ${ }^{1} \mathrm{H}$ NMR analysis (isolated yield: $58 \%$, $72.2 \mathrm{mg}$ ) from 4-ethynylbiphenyl (1p, $44.6 \mathrm{mg}, 0.25 \mathrm{mmol}) .6 \mathrm{wt} \%$ of 4-vinyl-1,1'-biphenyl is including in an isolated product.

${ }^{1} \mathbf{H}$ NMR $\left(\mathbf{C D C l}_{3}, \mathbf{5 0 0} \mathbf{M H z}\right): 7.60(\mathrm{dd}, 2 \mathrm{H}, J=7.1,1.5 \mathrm{~Hz}), 7.57(\mathrm{~d}, 2 \mathrm{H}, J=8.3 \mathrm{~Hz}), 7.49$ $(\mathrm{d}, 2 \mathrm{H}, J=8.3 \mathrm{~Hz}), 7.43(\mathrm{dd}, 2 \mathrm{H}, J=7.7,7.7 \mathrm{~Hz}), 7.34(\mathrm{tt}, 1 \mathrm{H}, J=7.3,1.2 \mathrm{~Hz}), 6.99-6.72(\mathrm{~m}, 2 \mathrm{H}), 1.60-1.50(\mathrm{~m}$, $6 \mathrm{H}), 1.35(\mathrm{tq}, 6 \mathrm{H}, J=7.4,7.2 \mathrm{~Hz}), 1.06-0.94(\mathrm{~m}, 6 \mathrm{H}), 0.91(\mathrm{t}, 9 \mathrm{H}, J=7.3 \mathrm{~Hz}) .{ }^{13} \mathbf{C} \mathbf{N M R}\left(\mathbf{C D C l}_{3}, 125 \mathrm{MHz}\right)$ : $145.7,130.1,128.9,127.5,127.38,127.35,127.1,127.0,126.8,126.6,29.3,27.5,13.9,9.8 . \mathbf{R}_{\mathbf{f}}=0.57$ (hexane). HRMS (APCI): $m / z:[\mathrm{M}+\mathrm{H}]^{+}$calcd for $\mathrm{C}_{26} \mathrm{H}_{39} \mathrm{Sn}$ 471.2068; Found 471.1991. ATR-FTIR (neat) v: 2954, 2924, $2850,1590,1486,1338,1173,985,751,694 \mathrm{~cm}^{-1}$.

\section{(E)-tributyl(4-methoxystyryl)stannane (3q)}

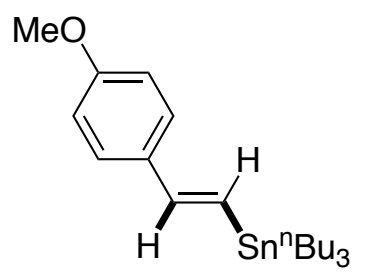

Using the Typical Procedure A (fluoride source: KF + 18-crown-6 instead of CsF, proton source: $\mathrm{H}_{2} \mathrm{O}$ instead of $\mathrm{MeOH}$, solvent: dioxane $1 \mathrm{~mL}$ ), the titled compound was obtained as a colorless oil in $83 \%$ NMR yield determined by ${ }^{1} \mathrm{H}$ NMR analysis from 1ethynyl-4-methoxybenzene (1q, $33.0 \mathrm{mg}, 0.25 \mathrm{mmol})$. The corresponding products (S3q) by Stille coupling reaction with iodobenzene are isolated instead of the stannylated products due to their instability.

${ }^{1} \mathrm{H}$ and ${ }^{13} \mathrm{C}$ NMR were in agreement with the reference ${ }^{2} .{ }^{1} \mathbf{H}$ NMR $\left(\mathbf{C D C l}_{3}, \mathbf{5 0 0} \mathbf{~ M H z}\right): 7.35(\mathrm{dd}, 2 \mathrm{H}, J=9.6,2.3$ $\mathrm{Hz}), 6.86(\mathrm{dd}, 2 \mathrm{H}, J=9.6,2.4 \mathrm{~Hz}), 6.84-6.79(\mathrm{~m}, 1 \mathrm{H}), 6.73-6.62(\mathrm{~m}, 1 \mathrm{H}), 3.81(\mathrm{~s}, 3 \mathrm{H}), 1.57-1.48(\mathrm{~m}, 6 \mathrm{H}), 1.37-$ $1.29(\mathrm{~m}, 6 \mathrm{H}), 0.99-0.87(\mathrm{~m}, 15 \mathrm{H}) . \mathbf{R}_{\mathbf{f}}=0.14$ (hexane). EI-MS: [M-Bu] $]^{+}$calced for $\mathrm{C}_{17} \mathrm{H}_{27} \mathrm{OSn} 367.1$; Found 367.1.

\section{(E)-1-methoxy-4-styrylbenzene (S3q)}

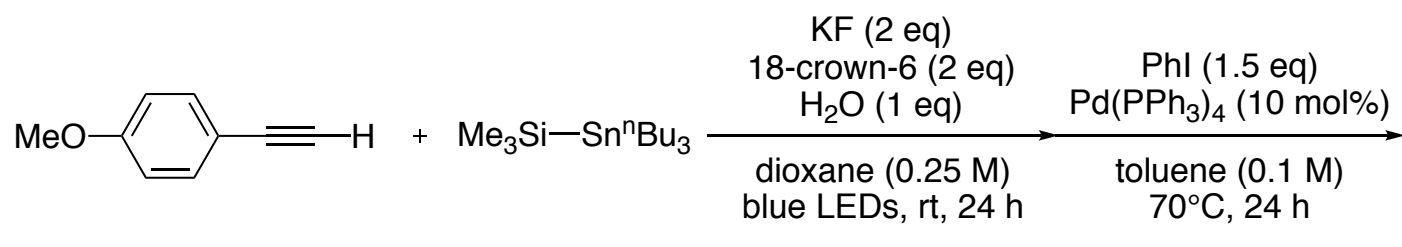

19

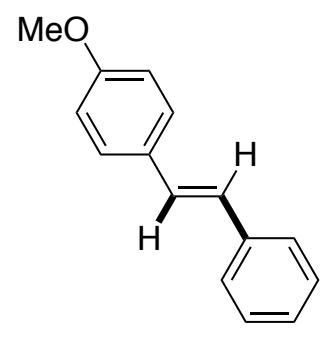

S3q

KF (26.1 mg, $0.50 \mathrm{mmol})$ and 18-crown-6 $(132 \mathrm{mg}, 0.50 \mathrm{mmol})$ were charged in a dried tube at argon glovebox and dissolved in dry dioxane $(1 \mathrm{~mL})$. To the mixture was successively added $\mathrm{H}_{2} \mathrm{O}(4.51 \mathrm{mg}, 0.250 \mathrm{mmol})$, trimethyl(tributylstannyl)silane $(\mathbf{2}, 182 \mathrm{mg}, 0.50 \mathrm{mmol})$ and 1-ethynyl-4-methoxybenzene $(\mathbf{1 q}, 33.0 \mathrm{mg}, 0.25$ $\mathrm{mmol})$ at room temperature. After the reaction tube was sealed and irradiated with a blue LED lamp, the solution was stirred for $24 \mathrm{~h}$ at room temperature. Then, after $\mathrm{Pd}\left(\mathrm{PPh}_{3}\right)_{4}(28.9 \mathrm{mg}, 0.025 \mathrm{mmol}), 4$-iodobenzene $(76.5 \mathrm{mg}$, $0.375 \mathrm{mmol})$, and toluene $(2.5 \mathrm{~mL})$ were successively added to the mixture, the resulting mixture was heated to 
$70^{\circ} \mathrm{C}$ and stirred for $24 \mathrm{~h}$. The reaction was quenched by $\mathrm{H}_{2} \mathrm{O}(1 \mathrm{~mL})$, and then extracted with AcOEt $(3 \mathrm{~mL} \times 3)$. The AcOEt layers were combined and dried over $\mathrm{MgSO}_{4}$, and the solvent was removed under reduced pressure. The residue was purified by silica gel column chromatography using hexane as eluent to give the titled compound (9q) as a white solid (isolated yield: 2 steps $71 \%, 37.1 \mathrm{mg}$ ).

${ }^{1} \mathrm{H}$ and ${ }^{13} \mathrm{C}$ NMR were in agreement with the reference ${ }^{5} .{ }^{1} \mathbf{H} \mathbf{~ N M R}\left(\mathbf{C D C l}_{3}, \mathbf{5 0 0} \mathbf{M H z}\right): 7.49(\mathrm{~d}, 2 \mathrm{H}, J=7.0 \mathrm{~Hz})$, $7.45(\mathrm{~d}, 2 \mathrm{H}, J=9.0 \mathrm{~Hz}), 7.34(\mathrm{dd}, 2 \mathrm{H}, J=9.0 \mathrm{~Hz}), 7.24-7.21(\mathrm{~m}, 1 \mathrm{H}), 7.11-6.93(\mathrm{~m}, 2 \mathrm{H}), 6.91-6.85(\mathrm{~m}, 2 \mathrm{H}), 3.83$ (s, 3H). ${ }^{13} \mathbf{C}$ NMR (CDCl 3 , 125 MHz): 137.8, 130.3, 128.7, 128.4, 127.9, 127.0, 126.8, 126.4, 114.4, 114.2, 56.1. $\mathbf{R}_{\mathbf{f}}=0.30$ (hexane:AcOEt =10:1). EI-MS: $m / z:[\mathrm{M}-\mathrm{Bu}]^{+}$calcd for $\mathrm{C}_{15} \mathrm{H}_{14} \mathrm{O} 210.1$; Found 210.1.

(E)-4-(2-(tributylstannyl)vinyl)aniline (3r)

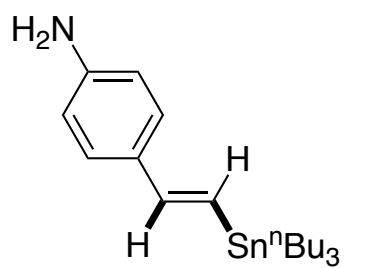

Using the Typical Procedure A (fluoride source: KF + 18-crown-6 instead of CsF, proton source: $\mathrm{H}_{2} \mathrm{O}$ instead of $\mathrm{MeOH}$, solvent: dioxane $1 \mathrm{~mL}$ ) the titled compound was obtained as a colorless oil in $83 \%$ NMR yield determined by ${ }^{1} \mathrm{H}$ NMR analysis from 4ethynylaniline (1r, $29 \mathrm{mg}, 0.25 \mathrm{mmol})$. The corresponding products (S3r) by Stille coupling reaction with iodobenzene are isolated instead of the stannylated products due to their instability.

${ }^{1} \mathbf{H}$ NMR $\left(\mathbf{C D C l}_{3}, \mathbf{5 0 0} \mathbf{M H z}\right): 7.24(\mathrm{~d}, 2 \mathrm{H}, J=8.4 \mathrm{~Hz}), 6.70-6.72(\mathrm{~m}, 1 \mathrm{H}), 6.64(\mathrm{~d}, 2 \mathrm{H}, J=8.7 \mathrm{~Hz}), 6.61-6.55(\mathrm{~m}$, $1 \mathrm{H}), 3.69(\mathrm{~s}, 2 \mathrm{H}), 1.54-1.46(\mathrm{~m}, 6 \mathrm{H}), 1.35-1.28(\mathrm{~m}, 6 \mathrm{H}), 1.00-0.87(\mathrm{~m}, 15 \mathrm{H}) . \mathbf{R}_{\mathbf{f}}=0.10$ (hexane:AcOEt $\left.=10: 1\right)$. EI-MS: $[\mathrm{M}-\mathrm{Bu}]^{+}$calced for $\mathrm{C}_{16} \mathrm{H}_{26} \mathrm{NSn} 352.1$; Found 352.1.

(E)-4-styrylaniline (S3r)

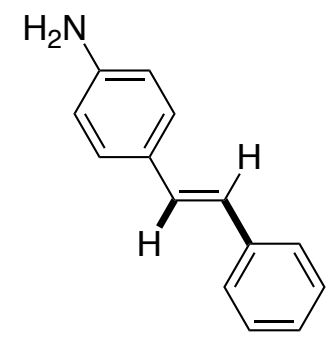

Using the procedure for the synthesis of $\mathbf{S 3 q}$, the titled compound was obtained as a brown solid (isolated yield: 2 steps 73\%, $35.8 \mathrm{mg}$ ) from 4-ethynylaniline (1r, $29.3 \mathrm{mg}, 0.25$ mmol).

${ }^{1} \mathrm{H}$ and ${ }^{13} \mathrm{C}$ NMR were in agreement with the reference ${ }^{5} .{ }^{1} \mathbf{H}$ NMR $\left(\mathbf{C D C l}_{3}, \mathbf{5 0 0} \mathbf{M H z}\right)$ : $7.47(\mathrm{~d}, 2 \mathrm{H}, J=7.3 \mathrm{~Hz}), 7.37-7.31(\mathrm{~m}, 4 \mathrm{H}), 7.21(\mathrm{dd}, 1 \mathrm{H}, J=7.3,7.3 \mathrm{~Hz}), 7.06-6.90(\mathrm{~m}$, $2 \mathrm{H}), 6.68(\mathrm{~d}, 2 \mathrm{H}, J=7.9 \mathrm{~Hz}), 3.75(\mathrm{~s}, 2 \mathrm{H}) .{ }^{13} \mathbf{C} \mathbf{~ N M R}\left(\mathbf{C D C l}_{3}, \mathbf{1 2 5} \mathbf{M H z}\right): 146.3,138.1$, 128.8, 128.7, 128.2, 127.9, 127.0, 126.2, 125.3, 115.4. $\mathbf{R}_{\mathbf{f}}=0.17$ (hexane:AcOEt =4:1).

EI-MS: $m / z:[\mathrm{M}-\mathrm{Bu}]^{+}$calcd for $\mathrm{C}_{14} \mathrm{H}_{13} \mathrm{~N}$ 195.1; Found 195.0.

\section{(E)-trimethyl(4-(2-(tributylstannyl)vinyl)phenyl)silane (3s)}

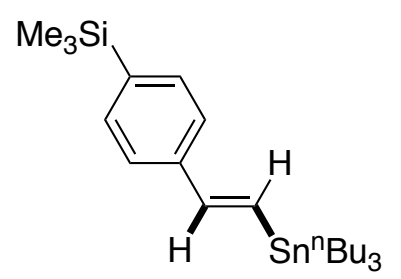

Using the Typical Procedure A (fluoride source: KF + 18-crown-6 instead of CsF, proton source: $\mathrm{H}_{2} \mathrm{O}$ instead of $\mathrm{MeOH}$, solvent: dioxane $1 \mathrm{~mL}$ ), the titled compound was obtained as a colorless oil in $>99 \%$ NMR yield determined by ${ }^{1} \mathrm{H}$ NMR analysis (isolated yield: $72 \%, 83.9 \mathrm{mg}$ ) from (4-ethynylphenyl)trimethylsilane (1s, $43.6 \mathrm{mg}$, $0.25 \mathrm{mmol})$.

${ }^{1} \mathbf{H}$ NMR $\left(\mathbf{C D C l}_{3}, \mathbf{5 0 0} \mathbf{M H z}\right): 7.48(\mathrm{~d}, 2 \mathrm{H}, J=8.2 \mathrm{~Hz}), 7.40(\mathrm{~d}, 2 \mathrm{H}, J=8.0 \mathrm{~Hz}), 6.95-$ $6.80(\mathrm{~m}, 2 \mathrm{H}), 1.59-1.50(\mathrm{~m}, 6 \mathrm{H}), 1.34(\mathrm{tq}, 6 \mathrm{H}, J=7.4,7.0 \mathrm{~Hz}), 1.04-0.93(\mathrm{~m}, 6 \mathrm{H}), 0.90,(\mathrm{t}, 9 \mathrm{H}, J=7.3 \mathrm{~Hz}), 0.26$ $(\mathrm{s}, 9 \mathrm{H}) .{ }^{13} \mathbf{C} \mathbf{N M R}\left(\mathbf{C D C l}_{3}, \mathbf{1 2 5} \mathbf{M H z}\right): 146.2,139.9,139.4,133.7,130.2,125.4,29.3,27.4,13.9,9.8,-1.0 . \mathbf{R}_{\mathrm{f}}=$ 0.68 (hexane). HRMS (APCI): $m / z:[\mathrm{M}-\mathrm{Bu}]^{+}$calcd for $\mathrm{C}_{19} \mathrm{H}_{33} \mathrm{SiSn} 409.1368$; Found 409.1327. ATR-FTIR (neat) v: $2954,2925,2850,1587,1463,1248,1107,985,833,749,690 \mathrm{~cm}^{-1}$. 
(E)-tributyl(4-butylstyryl)stannane (3t)

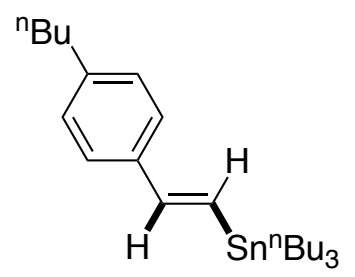

Using the Typical Procedure A (fluoride source: KF + 18-crown-6 instead of CsF, proton source: $\mathrm{H}_{2} \mathrm{O}$ instead of $\mathrm{MeOH}$, solvent: dioxane $1 \mathrm{~mL}$ ), the titled compound was obtained as a colorless oil in $81 \%$ NMR yield determined by ${ }^{1} \mathrm{H}$ NMR analysis (isolated yield: $41 \%$, $46.3 \mathrm{mg}$ ) from 1-butyl-4-ethynylbenzene (1t, $39.6 \mathrm{mg}, 0.25 \mathrm{mmol})$.

${ }^{1} \mathbf{H}$ NMR $\left(\mathbf{C D C l}_{3}, \mathbf{5 0 0} \mathbf{M H z}\right): 7.33(\mathrm{~d}, 2 \mathrm{H}, J=7.9 \mathrm{~Hz}), 7.13(\mathrm{~d}, 2 \mathrm{H}, J=7.9 \mathrm{~Hz}), 6.91-$ $6.73(\mathrm{~m}, 2 \mathrm{H}), 2.59(\mathrm{t}, 2 \mathrm{H}, J=7.7 \mathrm{~Hz}), 1.61-1.50(\mathrm{~m}, 8 \mathrm{H}), 1.38-1.31(\mathrm{~m}, 8 \mathrm{H}), 0.98-0.93$ (m, 6H), 0.93-0.88 (m, 12H). ${ }^{13} \mathbf{C} \mathbf{~ N M R}\left(\mathbf{C D C l}_{3}, \mathbf{1 2 5} \mathbf{M H z}\right):$ 146.1 142.6, 136.6, 128.7, 128.2, 126.0, 35.5, 33.8, 29.3, 27.5, 22.5, 14.1, 13.9, 9.7. $\mathbf{R}_{\mathbf{f}}=0.62$ (hexane). HRMS (APCI): $m / z:[\mathrm{M}-\mathrm{Bu}]^{+}$calcd for $\mathrm{C}_{20} \mathrm{H}_{33} \mathrm{Sn} 393.1599$; Found 393.1584. ATR-FTIR (neat) v: 2955, 2925, 2854, 1463, 1172, 1071, 985, 843, 670, $592 \mathrm{~cm}^{-1}$.

\section{(E)-(4-(2-(tributylstannyl)vinyl)phenyl)methanol (3u)}

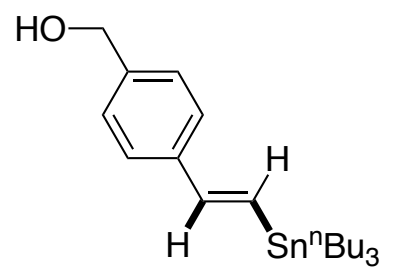

Using the Typical Procedure A (fluoride source: KF + 18-crown-6 instead of CsF, proton source: $\mathrm{H}_{2} \mathrm{O}$ instead of $\mathrm{MeOH}$, solvent: dioxane $1 \mathrm{~mL}$ ), the titled compound was obtained as a colorless oil in 59\% NMR yield determined by ${ }^{1} \mathrm{H}$ NMR analysis (isolated yield: $51 \%, 54.1 \mathrm{mg}$ ) from (4-ethynylphenyl)methanol (1u, $33.0 \mathrm{mg}, 0.25 \mathrm{mmol})$.

${ }^{1} \mathbf{H}$ NMR $\left(\mathbf{C D C l}_{3}, \mathbf{5 0 0} \mathbf{M H z}\right): 7.41(\mathrm{~d}, 1 \mathrm{H}, J=8.0 \mathrm{~Hz}), 7.32(\mathrm{~d}, 1 \mathrm{H}, J=8.0 \mathrm{~Hz}), 6.93$ $6.79(\mathrm{~m}, 2 \mathrm{H}), 4.67(\mathrm{~d}, 2 \mathrm{H}, J=5.5 \mathrm{~Hz}), 1.58-1.50(\mathrm{~m}, 6 \mathrm{H}), 1.33(\mathrm{tq}, 6 \mathrm{H}, J=7.4,7.1 \mathrm{~Hz})$, 1.02-0.93 (m, 6H), $0.90(\mathrm{t}, 9 \mathrm{H}, J=7.4 \mathrm{~Hz}) .{ }^{13} \mathbf{C}$ NMR $\left(\mathbf{C D C l}_{3}, \mathbf{1 2 5} \mathbf{M H z}\right): 145.7,140.2,138.5,130.0,127.3$, 126.3 , 65.4, 29.3 27.5, 13.9, 9.8. $\mathbf{R}_{\mathbf{f}}=0.33$ (hexane:AcOEt $=20: 1$ ). HRMS (APCI): $m / z:[\mathrm{M}-\mathrm{Bu}]^{+}$calcd for $\mathrm{C}_{17} \mathrm{H}_{27} \mathrm{OSn}$ 367.1078; Found 367.1080. ATR-FTIR (neat) v: 2955, 2923, 2850, 2030, 1742, 1589, 1463, 1239, $985,839,667 \mathrm{~cm}^{-1}$.

\section{(E)-tributyl(2-(thiophen-3-yl)vinyl)stannane (3v)}

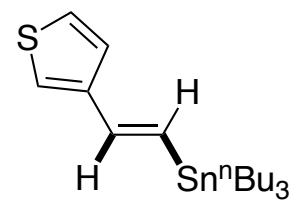

Using the Typical Procedure A (fluoride source: KF + 18-crown-6 instead of CsF, proton source: $\mathrm{H}_{2} \mathrm{O}$ instead of $\mathrm{MeOH}$, solvent: dioxane $1 \mathrm{~mL}$ ), the titled compound was obtained as a yellow oil in $88 \%$ NMR yield determined by ${ }^{1} \mathrm{H}$ NMR analysis (isolated yield: $44 \%$, 43.6mg) from 3-ethynylthiophene (1v, $27.0 \mathrm{mg}, 0.25 \mathrm{mmol})$.

${ }^{1} \mathrm{H}$ NMR $\left(\mathbf{C D C l}_{3}, \mathbf{5 0 0} \mathbf{M H z}\right)$ : 7.29-7.26 (m, 1H), 7.26-7.23 (m, 1H), $7.13(\mathrm{~s}, 1 \mathrm{H})$, 6.95-6.78 $(\mathrm{m}, 1 \mathrm{H}), 6.70-6.51(\mathrm{~m}, 1 \mathrm{H}), 1.58-1.50(\mathrm{~m}, 6 \mathrm{H}), 1.33(\mathrm{tq}, 6 \mathrm{H}, J=7.4,7.1 \mathrm{~Hz}), 1.02-0.93(\mathrm{~m}, 6 \mathrm{H}), 0.90(\mathrm{t}, 9 \mathrm{H}, J=$ $7.4 \mathrm{~Hz}) .{ }^{13} \mathbf{C} \mathbf{N M R}\left(\mathbf{C D C l}_{3}, \mathbf{1 2 5} \mathbf{~ M H z}\right): 142.8,140.1,129.2,125.8,125.0,121.6,29.2,27.5,13.9,9.7 . \mathbf{R}_{\mathbf{f}}=0.61$ (hexane). HRMS (APCI): $m / z$ : [M-Bu] $]^{+}$calcd for $\mathrm{C}_{14} \mathrm{H}_{23} \mathrm{SSn} 343.0537$; Found 343.0537. ATR-FTIR (neat) v: $2954,2923,2850,1587,1463,1376,1071,982,863,747 \mathrm{~cm}^{-1}$.

\section{(E)-tributyl(2-(thiophen-2-yl)vinyl)stannane (3w)}

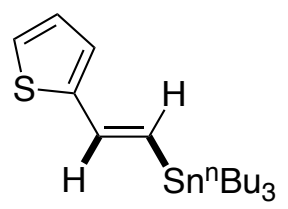

Using the Typical Procedure A (fluoride source: KF + 18-crown-6 instead of CsF, proton source: $\mathrm{H}_{2} \mathrm{O}$ instead of $\mathrm{MeOH}$, solvent: dioxane $1 \mathrm{~mL}$ ), the titled compound was obtained as a yellow oil in 60\% NMR yield determined by ${ }^{1} \mathrm{H}$ NMR analysis (isolated yield: $28 \%, 38.6 \mathrm{mg}$ ) from 2-ethynylthiophene (1w, $27.0 \mathrm{mg}, 0.25 \mathrm{mmol}) .25 \mathrm{wt} \%$ of hexa- $n$-butyl-distannane $\left({ }^{\mathrm{n}} \mathrm{Bu}_{3} \mathrm{Sn}-\mathrm{Sn}^{\mathrm{n}} \mathrm{Bu}_{3}\right)$ is including in an isolated product.

${ }^{1} \mathbf{H}$ NMR $\left(\mathbf{C D C l}_{3}, \mathbf{5 0 0} \mathbf{M H z}\right):$ 7.15-7.13 (m, 1H), 6.98-6.90 (m, 3H), 6.67-6.49 (m, 1H), 1.33 (tq, 6H, J = 7.4, 7.1 $\mathrm{Hz}), 1.02-0.93(\mathrm{~m}, 6 \mathrm{H}), 0.90(\mathrm{t}, 9 \mathrm{H}, J=7.4 \mathrm{~Hz}) .{ }^{13} \mathbf{C} \mathbf{N M R}\left(\mathbf{C D C l}_{3}, \mathbf{1 2 5} \mathbf{M H z}\right):$ 146.0, 138.5, 129.6, 127.3, 124.3, 124.1, 29.1, 27.3 , 13.7, 9.6. $\mathbf{R}_{\mathbf{f}}=0.61$ (hexane). HRMS (APCI): $m / z:[\mathrm{M}-\mathrm{Bu}]^{+}$calcd for $\mathrm{C}_{14} \mathrm{H}_{23} \mathrm{SSn} 343.0537$; Found 343.0520. ATR-FTIR (neat) v: 2954, 2923, 2852, 1578, 1455, 1072, 974, 755, 691, $596 \mathrm{~cm}^{-1}$. 
(E)-3-(2-(tributylstannyl)vinyl)pyridine (3x)

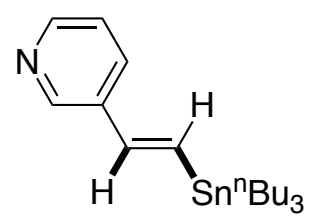

Using the Typical Procedure A (fluoride source: KF + 18-crown-6 instead of CsF, proton source: $\mathrm{H}_{2} \mathrm{O}$ instead of $\mathrm{MeOH}$, solvent: dioxane $1 \mathrm{~mL}$ ), the titled compound was obtained as a yellow oil in $77 \%$ NMR yield determined by ${ }^{1} \mathrm{H}$ NMR analysis (isolated yield: $87 \%$, $85.4 \mathrm{mg}$ ) from 3-ethynylpyridine (1x, $25.8 \mathrm{mg}, 0.25 \mathrm{mmol})$.

${ }^{1} \mathbf{H}$ NMR $\left(\mathbf{C D C l}_{3}, \mathbf{5 0 0} \mathbf{M H z}\right): 8.60(\mathrm{dd}, 1 \mathrm{H}, J=2.0,2.0 \mathrm{~Hz}), 8.45(\mathrm{ddd}, 1 \mathrm{H}, J=4.8,1.6$, $1.6 \mathrm{~Hz}), 7.72(\mathrm{dd}, 1 \mathrm{H}, J=8.0,1.9 \mathrm{~Hz}), 7.26-7.21(\mathrm{~m}, 1 \mathrm{H}), 7.07-6.76(\mathrm{~m}, 2 \mathrm{H}), 1.60-1.51(\mathrm{~m}, 6 \mathrm{H}), 1.34(\mathrm{tq}, 6 \mathrm{H}, J$ $=7.4,7.2 \mathrm{~Hz}), 1.02-0.95(\mathrm{~m}, 6 \mathrm{H}), 0.91(\mathrm{t}, 9 \mathrm{H}, J=7.3 \mathrm{~Hz}) .{ }^{13} \mathbf{C} \mathbf{~ N M R}\left(\mathbf{C D C l}_{3}, \mathbf{1 2 5} \mathbf{~ M H z}\right): 149.0,148.0,142.6$, $142.4,134.2,133.6,132.4,29.2,27.4,13.8,9.8 . \mathbf{R}_{\mathbf{f}}=0.07$ (hexane). HRMS (ESI): $\mathrm{m} / z:[\mathrm{M}+\mathrm{H}]^{+}$calcd for $\mathrm{C}_{19} \mathrm{H}_{33} \mathrm{NSn} 396.1708$, found 396.1710. ATR-FTIR (neat) v: 2955, 2923, 2850, 1590, 1463, 1192, 1072, 986, 873, $687 \mathrm{~cm}^{-1}$.

\section{(E)-2-(2-(tributylstannyl)vinyl)pyridine (3y)}

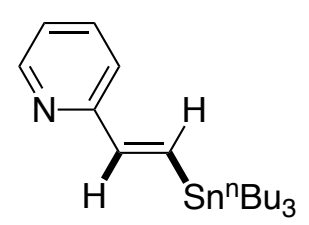

Using the Typical Procedure A (fluoride source: KF + 18-crown-6 instead of CsF, proton source: $\mathrm{H}_{2} \mathrm{O}$ instead of $\mathrm{MeOH}$, solvent: dioxane $1 \mathrm{~mL}$ ), the titled compound was obtained as a yellow oil in 47\% NMR yield determined by ${ }^{1} \mathrm{H}$ NMR analysis (isolated yield: $39 \%, 38.4$ $\mathrm{mg}$ ) from 2-ethynylpyridine (1y, $25.8 \mathrm{mg}, 0.25 \mathrm{mmol})$.

${ }^{1} \mathbf{H}$ NMR $\left(\mathbf{C D C l}_{3}, \mathbf{5 0 0} \mathbf{M H z}\right): 8.43(\mathrm{~d}, 1 \mathrm{H}, J=3.6 \mathrm{~Hz}), 7.62(\mathrm{dd}, 1 \mathrm{H}, J=7.6,7.6 \mathrm{~Hz}), 7.58$ $7.28(\mathrm{~m}, 1 \mathrm{H}), 7.14-7.08(\mathrm{~m}, 2 \mathrm{H}), 6.72-6.53(\mathrm{~m}, 1 \mathrm{H}), 1.51-1.42(\mathrm{~m}, 6 \mathrm{H}), 1.27$ (tq, $6 \mathrm{H}, J=7.3,7.2 \mathrm{~Hz}), 0.92-0.80$ $(\mathrm{m}, 15 \mathrm{H}) .{ }^{13} \mathbf{C}$ NMR $\left(\mathbf{C D C l}_{3}, \mathbf{1 2 5} \mathbf{M H z}\right): 155.8,148.1,142.2,141.4,136.7,123.7,122.2,29.5,27.6,13.9,12.7$. $\mathbf{R}_{\mathbf{f}}=0.21$ (hexane). HRMS (ESI): $\mathrm{m} / z:[\mathrm{M}+\mathrm{H}]^{+}$calcd for $\mathrm{C}_{19} \mathrm{H}_{33} \mathrm{NSn}$ 396.1708; Found 396.1715. ATR-FTIR (neat) v: 2953, 2918, 2870, 1583, 1427, 1298, 1148, 873, 806, $682 \mathrm{~cm}^{-1}$.

\section{Tributyl(phenethyl)stannane (5c)}

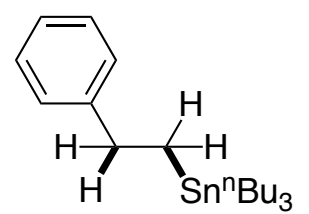

Using the Typical Procedure A (fluoride source: KF + 18-crown-6 instead of CsF, proton source: ${ }^{\mathrm{BuOH}}$ instead of $\mathrm{MeOH}$, solvent: $\mathrm{DMF}(0.5 \mathrm{~mL})$ instead of dioxane), the titled compound was obtained as a colorless oil in $85 \%$ NMR yield determined by ${ }^{1} \mathrm{H}$ NMR analysis (isolated yield: $36 \%, 35.5 \mathrm{mg}$ ) from styrene $(\mathbf{4 a}, 26.0 \mathrm{mg}, 0.25 \mathrm{mmol})$.

${ }^{1} \mathbf{H}$ NMR $\left(\mathbf{C D C l}_{3}, \mathbf{5 0 0} \mathbf{M H z}\right): 7.30-7.26(\mathrm{~m}, 2 \mathrm{H}), 7.20(\mathrm{~d}, 2 \mathrm{H}, J=7.1 \mathrm{~Hz}), 7.16(\mathrm{t}, 1 \mathrm{H}, J=$ $7.2 \mathrm{~Hz}), 2.87-2.74(\mathrm{~m}, 2 \mathrm{H}), 1.48-1.41(\mathrm{~m}, 6 \mathrm{H}), 1.29(\mathrm{tq}, 6 \mathrm{H}, J=7.4,7.2 \mathrm{~Hz}), 1.18-1.08(\mathrm{~m}, 2 \mathrm{H}), 0.89(\mathrm{t}, 9 \mathrm{H}, J=$ 7.3 Hz), 0.85-0.74 (m, 6H). ${ }^{13} \mathbf{C}$ NMR (CDCl 3 , 125 MHz): 146.1, 128.5, 127.9, 125.7, 33.2, 29.4, 27.5, 13.9, 10.9, 9.0. $\mathbf{R}_{\mathbf{f}}=0.80$ (hexane). HRMS (APCI): $\mathrm{m} / \mathrm{z}:[\mathrm{M}-\mathrm{Bu}]^{+}$calcd for $\mathrm{C}_{16} \mathrm{H}_{27} \mathrm{Sn} 339.1129$; Found 339.1130. ATR-FTIR (neat) v: 2955, 2923, 2851, 1602, 1454, 1376, 1070, 960, 874, $696 \mathrm{~cm}^{-1}$. 


\subsection{Dehalostannylation of Aryl Halides (Table 4)}

Typical Procedure B:

[1,1'-biphenyl]-4-yltributylstannane (12a)

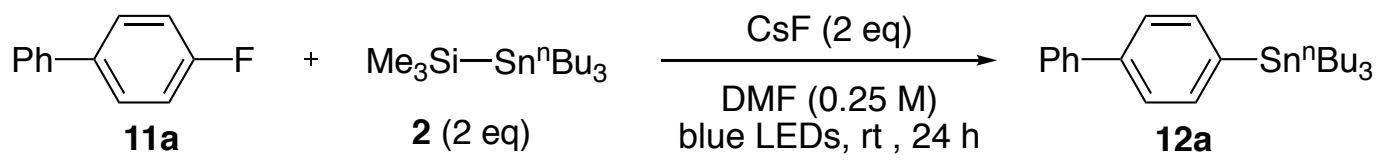

CsF (76.0 mg, $0.50 \mathrm{mmol}$ ) was charged in a dried tube at argon glovebox and dissolved in dry DMF (1 mL). To the mixture was successively added trimethyl(tributylstannyl)silane (2, $182 \mathrm{mg}, 0.50 \mathrm{mmol})$ and 4-fluoro-1,1'biphenyl (11a, $43.0 \mathrm{mg}, 0.25 \mathrm{mmol}$ ) at room temperature. After the reaction tube was sealed and irradiated with a blue LED lamp, the solution was stirred for $24 \mathrm{~h}$ at room temperature. The reaction was quenched by $\mathrm{H}_{2} \mathrm{O}(1 \mathrm{~mL})$, and then extracted with AcOEt $(3 \mathrm{~mL} \times 3)$. The AcOEt layers were combined and dried over $\mathrm{MgSO}_{4}$, and the solvent was removed under reduced pressure. The residue was purified by silica gel column chromatography using hexane as eluent to give the titled compound (12a) as a colorless oil in 78\% NMR yield determined by ${ }^{1} \mathrm{H}$ NMR analysis (isolated yield: $50 \%, 55.4 \mathrm{mg}$ ).

${ }^{1} \mathrm{H}$ and ${ }^{13} \mathrm{C}$ NMR were in agreement with the reference ${ }^{6} .{ }^{1} \mathbf{H} \mathbf{~ N M R}\left(\mathbf{C D C l}_{3}, \mathbf{5 0 0} \mathbf{~} \mathbf{H H z}\right): 7.61(\mathrm{dd}, 2 \mathrm{H}, J=8.3,1.2$ $\mathrm{Hz}), 7.56(\mathrm{dd}, 4 \mathrm{H}, J=11.8,8.2 \mathrm{~Hz}), 7.44(\mathrm{dd}, 2 \mathrm{H}, J=7.7,7.7 \mathrm{~Hz}), 7.34(\mathrm{tt}, 1 \mathrm{H}, J=7.4,1.8 \mathrm{~Hz}), 1.61-1.54(\mathrm{~m}$, 6H), $1.36(\mathrm{tq}, 6 \mathrm{H}, J=7.4,7.2 \mathrm{~Hz}), 1.14-1.04(\mathrm{~m}, 6 \mathrm{H}), 0.91(\mathrm{t}, 9 \mathrm{H}, J=7.3 \mathrm{~Hz}) .{ }^{13} \mathbf{C} \mathbf{N M R}\left(\mathbf{C D C l}_{3}, \mathbf{1 2 5} \mathbf{~ M H z}\right)$ : $141.5,141.0,140.9$, 137.0, 128.9, 127.3, 127.2, 126.8, 29.3, 27.5, 13.8, 9.8. $\mathbf{R}_{\mathbf{f}}=0.57$ (hexane). EI-MS: $\mathrm{m} / \mathrm{z}:[\mathrm{M}-$ $\mathrm{Bu}]^{+}$calcd for $\mathrm{C}_{20} \mathrm{H}_{27} \mathrm{Sn} 387.1$; Found 387.1.

\section{[1,1'-biphenyl]-2-yltributylstannane (12b)}

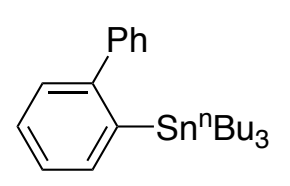

Using the Typical Procedure B, the titled compound was obtained as a colorless oil in 56\% NMR yield determined by ${ }^{1} \mathrm{H}$ NMR analysis (isolated yield: $48 \%, 52.8 \mathrm{mg}$ ) from 2-fluoro1,1'-biphenyl (11b, $43.0 \mathrm{mg}, 0.25 \mathrm{mmol})$.

${ }^{1} \mathrm{H}$ and ${ }^{13} \mathrm{C}$ NMR were in agreement with the reference ${ }^{6} .{ }^{1} \mathbf{H}$ NMR $\left(\mathbf{C D C l}_{3}, \mathbf{5 0 0} \mathbf{M H z}\right):$ 7.597.50 (m, 1H), 7.43-7.29 (m, 8H), 1.41-1.29 (m, 6H), 1.23 (tq, 6H, J = 7.3, 7.2 Hz), 0.83 (t, 9H, J = 7.2 Hz), 0.79$0.65(\mathrm{~m}, 6 \mathrm{H}) .{ }^{13} \mathbf{C} \mathbf{~ N M R}\left(\mathbf{C D C l}_{3}, \mathbf{1 2 5} \mathbf{~ M H z}\right): 150.6,145.9,142.1,137.1,128.92,128.88,128.3,128.1,127.1$, $126.4,29.1,27.5,13.8,10.9 . \mathbf{R}_{\mathbf{f}}=0.67$ (hexane). EI-MS: $m / z:[\mathrm{M}-\mathrm{Bu}]^{+}$calcd for $\mathrm{C}_{20} \mathrm{H}_{27} \mathrm{Sn} 387.1$; Found 387.1 .

\section{2-(4-(tributylstannyl)phenyl)pyridine (12c)}

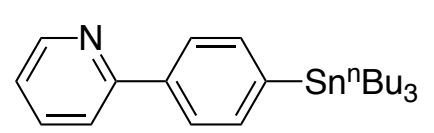

Using the Typical Procedure B, the titled compound was obtained as a colorless oil in 56\% NMR yield determined by ${ }^{1} \mathrm{H}$ NMR analysis (isolated yield: $54 \%, 60.0 \mathrm{mg}$ ) from 2-(4-fluorophenyl)pyridine (11c, $43.3 \mathrm{mg}, 0.25 \mathrm{mmol})$.

${ }^{1} \mathbf{H}$ NMR $\left(\mathbf{C D C l}_{3}, \mathbf{5 0 0} \mathbf{M H z}\right): 8.69(\mathrm{dd}, 1 \mathrm{H}, J=4.6,1.3 \mathrm{~Hz}), 7.93(\mathrm{~d}, 2 \mathrm{H}, J=8.1 \mathrm{~Hz}), 7.76-7.71(\mathrm{~m}, 2 \mathrm{H}), 7.63-$ $7.52(\mathrm{~m}, 2 \mathrm{H}), 7.24-7.19(\mathrm{~m}, 1 \mathrm{H}), 1.60-1.52(\mathrm{~m}, 6 \mathrm{H}), 1.34(\mathrm{tq}, 6 \mathrm{H}, J=7.7,7.3 \mathrm{~Hz}), 1.16-1.06(\mathrm{~m}, 6 \mathrm{H}), 0.89(\mathrm{t}, 9 \mathrm{H}$, $J=7.3 \mathrm{~Hz}) .{ }^{13} \mathbf{C} \mathbf{N M R}\left(\mathbf{C D C l}_{3}, \mathbf{1 2 5} \mathbf{M H z}\right): 157.9,149.8,143.5,139.1,137.0,136.8,126.3,122.1,120.6,29.2$, 27.5, 13.8, 9.8. $\mathbf{R}_{\mathbf{f}}=0.20$ (hexane:AcOEt $=10: 1$ ). HRMS (ESI): $m / z:[\mathrm{M}+\mathrm{H}]^{+}$Calcd for $\mathrm{C}_{23} \mathrm{H}_{36} \mathrm{NSn}$ 446.1864; Found 446.1869. ATR-FTIR (neat) v: 2955, 2923, 2851, 1585, 1464, 1247, 1151, 1013, 770, $655 \mathrm{~cm}^{-1}$.

\section{Tributyl(4-(thiophen-2-yl)phenyl)stannane (12d)}

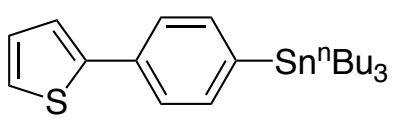

Using the Typical Procedure B, the titled compound was obtained as a colorless oil in $76 \%$ NMR yield determined by ${ }^{1} \mathrm{H}$ NMR analysis (isolated yield: $29 \%, 33.1 \mathrm{mg}$ ) from 2-(4-fluorophenyl)thiophene (11d, $44.6 \mathrm{mg}, 0.25 \mathrm{mmol})$. 
${ }^{1} \mathbf{H}$ NMR $\left(\mathbf{C D C l}_{3}, \mathbf{5 0 0} \mathbf{M H z}\right): 7.57(\mathrm{~d}, 2 \mathrm{H}, J=8.0 \mathrm{~Hz}), 7.47(\mathrm{~d}, 2 \mathrm{H}, J=8.0 \mathrm{~Hz}), 7.32(\mathrm{dd}, 1 \mathrm{H}, J=3.5,1.0 \mathrm{~Hz})$, $7.26(\mathrm{~d}, 1 \mathrm{H}, J=1.0 \mathrm{~Hz}), 7.07(\mathrm{dd}, 1 \mathrm{H}, J=5.1,3.6 \mathrm{~Hz}), 1.59-1.51(\mathrm{~m}, 6 \mathrm{H}), 1.34(\mathrm{tq}, 6 \mathrm{H}, J=7.4,7.3 \mathrm{~Hz}), 1.11-$ $1.03(\mathrm{~m}, 6 \mathrm{H}), 0.90(\mathrm{t}, 9 \mathrm{H}, J=7.3 \mathrm{~Hz}) .{ }^{13} \mathbf{C} \mathbf{~ N M R}\left(\mathbf{C D C l}_{3}, \mathbf{1 2 5} \mathbf{M H z}\right): 144.9,141.7,137.1,134.1,128.1,125.5$, 124.7, 123.0, 29.2, 27.5, 13.8, 9.8. $\mathbf{R}_{\mathbf{f}}=0.52$ (hexane). HRMS (APCI): $m / z:[\mathrm{M}+\mathrm{H}]^{+}$calcd for $\mathrm{C}_{22} \mathrm{H}_{35} \mathrm{SSn}$ 451.1476; Found 451.1403. ATR-FTIR (neat) v: 2954, 2924, 2851, 1588, 1463, 1257, 1072, 957, 806, $690 \mathrm{~cm}^{-1}$.

\section{Ethyl 4-(tributylstannyl)benzoate (12e)}

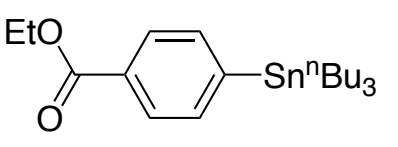

Using the Typical Procedure B ( $\mathrm{CsF}$ (0.4 eq) and 2 (4 eq) were used), the titled compound was obtained as a yellow oil in $83 \%$ NMR yield determined by ${ }^{1} \mathrm{H}$ NMR analysis (isolated yield: 63\%, $69.1 \mathrm{mg}$ ) from ethyl 4-fluorobenzoate (11e, $42.0 \mathrm{mg}$, $0.25 \mathrm{mmol})$.

${ }^{1} \mathrm{H}$ and ${ }^{13} \mathrm{C}$ NMR were in agreement with the reference ${ }^{7} .{ }^{1} \mathbf{H}$ NMR $\left(\mathbf{C D C l}_{3}, \mathbf{5 0 0} \mathbf{M H z}\right):$ 7.98-7.94 (m, 2H), 7.59$7.49(\mathrm{~m}, 2 \mathrm{H}), 4.37(\mathrm{q}, 2 \mathrm{H}, J=7.2 \mathrm{~Hz}), 1.57-1.50(\mathrm{~m}, 6 \mathrm{H}), 1.39(\mathrm{t}, 3 \mathrm{H}, J=7.1 \mathrm{~Hz}), 1.32(\mathrm{tq}, 6 \mathrm{H}, J=7.3,7.3 \mathrm{~Hz})$, 1.13-1.02 (m, 6H), $0.88(\mathrm{t}, 9 \mathrm{H}, J=7.3 \mathrm{~Hz}) .{ }^{13} \mathbf{C} \mathbf{N M R}\left(\mathbf{C D C l}_{3}, \mathbf{1 2 5} \mathbf{M H z}\right):$ 167.2, 149.6, 136.5, 130.1, 128.5, 61.0, $29.2,27.5,14.5,13.8,9.8 . \mathbf{R}_{\mathbf{f}}=0.33$ (hexane:AcOEt $=20: 1$ ). EI-MS: $m / z:[\mathrm{M}-\mathrm{Bu}]^{+}$calcd for $\mathrm{C}_{17} \mathrm{H}_{27} \mathrm{O}_{2} \mathrm{Sn} 383.1$; Found 383.2 .

\section{Methyl 4-(tributylstannyl)benzoate (12f)}

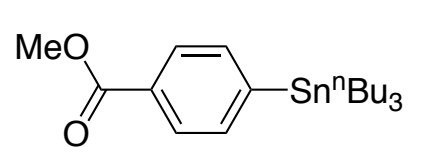

Using the Typical Procedure B (CsF (0.4 eq) and 2 (4 eq) were used), the titled compound was obtained as a colorless oil in $88 \%$ NMR yield determined by ${ }^{1} \mathrm{H}$ NMR analysis (isolated yield: 65\%, $69.4 \mathrm{mg}$ ) from methyl 4-fluorobenzoate (11f, $38.5 \mathrm{mg}$, $0.25 \mathrm{mmol})$.

${ }^{1} \mathrm{H}$ and ${ }^{13} \mathrm{C}$ NMR were in agreement with the reference ${ }^{6} .{ }^{1} \mathbf{H}$ NMR $\left(\mathbf{C D C l}_{3}, \mathbf{5 0 0} \mathbf{~ M H z}\right):$ 7.98-7.92 (m, 2H), 7.59$7.50(\mathrm{~m}, 2 \mathrm{H}), 3.90(\mathrm{~s}, 3 \mathrm{H}), 1.56-1.51(\mathrm{~m}, 6 \mathrm{H}), 1.32(\mathrm{tq}, 6 \mathrm{H}, J=7.4,7.3 \mathrm{~Hz}), 1.13-1.04(\mathrm{~m}, 6 \mathrm{H}), 0.88(\mathrm{t}, 9 \mathrm{H}, J=$ $7.2 \mathrm{~Hz}) .{ }^{13} \mathbf{C} \mathbf{N M R}\left(\mathbf{C D C l}_{3}, \mathbf{1 2 5} \mathbf{M H z}\right): 167.7,149.8,136.6,129.7,128.5,52.1,29.2,27.5,13.8,9.8 . \mathbf{R}_{\mathbf{f}}=0.37$ (hexane:AcOEt = 20:1). EI-MS: $m / z:[\mathrm{M}-\mathrm{Bu}]^{+}$calcd for $\mathrm{C}_{16} \mathrm{H}_{25} \mathrm{O}_{2} \mathrm{Sn} 369.1$; Found 369.0.

\section{Tributyl(4-(trifluoromethyl)phenyl)stannane (12g)}

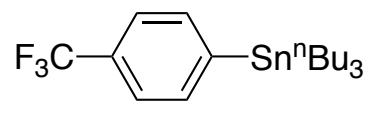

Using the Typical Procedure B $(\mathrm{CsF}(0.4 \mathrm{eq})$ and $\mathbf{2}(4 \mathrm{eq})$ were used), and the reaction time is $3 \mathrm{~h}$ ), the titled compound was obtained as a colorless oil in 51\% NMR yield determined by ${ }^{1} \mathrm{H}$ NMR analysis (isolated yield: $53 \%, 58.5 \mathrm{mg}$ ) from 1-fluoro-4(trifluoromethyl)benzene (11g, $41.0 \mathrm{mg}, 0.25 \mathrm{mmol}$ ). Using the Typical Procedure B (fluoride source: KF and 18-crown-6, solvent: THF), the titled compound was obtained in $72 \%$ NMR yield determined by ${ }^{1} \mathrm{H}$ NMR analysis from 1-iodo-4-(trifluoromethyl)benzene $(\mathbf{1 3 g}, 68.0 \mathrm{mg}, 0.25 \mathrm{mmol})$.

${ }^{1} \mathrm{H}$ and ${ }^{13} \mathrm{C}$ NMR were in agreement with the reference ${ }^{6} .{ }^{1} \mathbf{H}$ NMR $\left(\mathbf{C D C l}_{3}, \mathbf{5 0 0} \mathbf{M H z}\right): 7.58(\mathrm{~d}, 2 \mathrm{H}, J=7.8 \mathrm{~Hz})$, 7.56-7.53 (m, 2H), 1.57-1.51 (m, 6H), $1.33(\mathrm{tq}, 6 \mathrm{H}, J=7.4,7.1 \mathrm{~Hz}), 1.11-1.06(\mathrm{~m}, 6 \mathrm{H}), 0.89(\mathrm{t}, 9 \mathrm{H}, J=7.5 \mathrm{~Hz})$. ${ }^{13} \mathbf{C ~ N M R}\left(\mathbf{C D C l}_{3}, \mathbf{1 2 5} \mathbf{M H z}\right): 147.8,136.7(J=15.0 \mathrm{~Hz}), 130.3(J=32.5 \mathrm{~Hz}), 125.6(J=282.5 \mathrm{~Hz}), 124.3(J=$ $3.8 \mathrm{~Hz}), 29.2,27.5,13.8,9.8 .{ }^{19} \mathbf{F} \mathbf{N M R}\left(\mathbf{C D C l}_{3}, \mathbf{3 7 6} \mathbf{M H z}\right):-62.80 . \mathbf{R}_{\mathbf{f}}=0.82$ (hexane). EI-MS: $m / z:[\mathrm{M}-\mathrm{Bu}]^{+}$ calcd for $\mathrm{C}_{15} \mathrm{H}_{22} \mathrm{~F}_{3} \mathrm{Sn} 379.1$; Found 379.0.

\section{Tributyl(4-(3,3,4,4-tetramethyl-113,2,5-bromadioxolan-1-yl)phenyl)stannane (12h)}

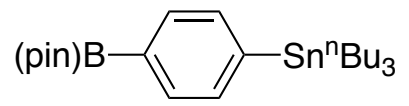

$0.25 \mathrm{mmol})$.
Using the Typical Procedure B, the titled compound was obtained as a colorless oil in $53 \%$ yield NMR yield determined by ${ }^{1} \mathrm{H}$ NMR analysis (isolated yield: $15 \%, 20.8$ $\mathrm{mg}$ ) from 2-(4-fluorophenyl)-4,4,5,5-tetramethyl-1,3,2-dioxaborolane (11h, $55.5 \mathrm{mg}$, 
${ }^{1} \mathbf{H}$ NMR (CDCl $\left.\mathbf{3}, \mathbf{5 0 0} \mathbf{M H z}\right)$ : 7.76-7.71 (m, 2H), 7.53-7.42 (m, 2H), 1.56-1.48 (m, 6H), 1.36-1.28 (m, 18H), 1.12$0.99(\mathrm{~m}, 6 \mathrm{H}), 0.87$ (t, 9H, $J=7.3 \mathrm{~Hz}) .{ }^{13} \mathbf{C} \mathbf{~ N M R}\left(\mathbf{C D C l}_{3}, \mathbf{1 2 5} \mathbf{M H z}\right): 146.7,136.1,134.0,83.8,29.2,27.5,25.0$, 13.8, 9.7. The carbon directly attached to the boron atom was not detected, likely due to quadropolar relaxation. ${ }^{11} \mathbf{B}$ NMR (CDCl3, 160 MHz): 32.2. $\mathbf{R}_{\mathbf{f}}=0.35$ (hexane : AcOEt = 10:1). HRMS (APCI): $\mathrm{m} / z:[\mathrm{M}+\mathrm{H}]^{+}$calcd for $\mathrm{C}_{24} \mathrm{H}_{44} \mathrm{BO}_{2} \mathrm{Sn}$ 495.2451; Found 495.2441. ATR-FTIR (neat) v: 2956, 2925, 2853, 1594, 1463, 1359, 1144, 962 , $860,657 \mathrm{~cm}^{-1}$.

\section{2-(tributylstannyl)benzonitrile (12i)}

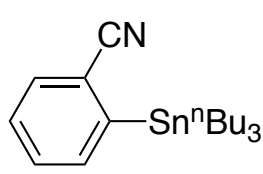

Using the Typical Procedure B, $(\mathrm{CsF}$ ( 0.4 eq) and 2 (4 eq) were used, and the reaction time is $3 \mathrm{~h}$ ), the titled compound was obtained as a colorless oil in $54 \%$ NMR yield determined by ${ }^{1} \mathrm{H}$ NMR analysis (isolated yield: $25 \%, 24.3 \mathrm{mg}$ ) from 2-fluorobenzonitrile (11i, $30.3 \mathrm{mg}$, $0.25 \mathrm{mmol})$.

${ }^{1} \mathrm{H}$ and ${ }^{13} \mathrm{C}$ NMR were in agreement with the reference ${ }^{7} .{ }^{1} \mathbf{H}$ NMR $\left(\mathbf{C D C l}_{3}, \mathbf{5 0 0} \mathbf{M H z}\right): 7.67-7.63(\mathrm{~m}, 1 \mathrm{H}), 7.58$ $7.51(\mathrm{~m}, 1 \mathrm{H}), 7.51-7.46(\mathrm{~m}, 1 \mathrm{H}), 7.39-7.34(\mathrm{~m}, 1 \mathrm{H}), 1.59-1.52(\mathrm{~m}, 6 \mathrm{H}), 1.34(\mathrm{tq}, 6 \mathrm{H}, J=7.4,7.1 \mathrm{~Hz}), 1.27-1.17$ $(\mathrm{m}, 6 \mathrm{H}), 0.87$ (t, 9H, $J=7.3 \mathrm{~Hz}) .{ }^{13} \mathbf{C} \mathbf{~ N M R}\left(\mathbf{C D C l}_{3}, \mathbf{1 2 5} \mathbf{M H z}\right): 148.5,137.1,133.1,131.5,128.4,120.8,120.4$, 29.1, 27.4, 13.8, 10.2. $\mathbf{R}_{\mathbf{f}}=0.38$ (hexane). EI-MS: $m / z:[\mathrm{M}-\mathrm{Bu}]^{+}$calcd for $\mathrm{C}_{15} \mathrm{H}_{22} \mathrm{NSn} 336.1$; Found 336.0.

\section{Tributyl(p-tolyl)stannane (12j)}

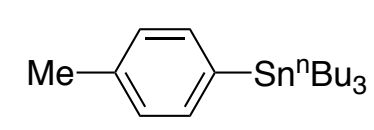

Using the Typical Procedure B, the titled compound was obtained as a colorless oil in $30 \%$ NMR yield determined by ${ }^{1} \mathrm{H}$ NMR analysis (isolated yield: $17 \%, 15.9 \mathrm{mg}$ ) from 1-fluoro-4-methylbenzene (11j, $27.5 \mathrm{mg}, 0.25 \mathrm{mmol})$. Using the Typical Procedure B (fluoride source: KF and 18-crown-6, solvent: THF), the titled compound was obtained in 75\% NMR yield determined by ${ }^{1} \mathrm{H}$ NMR analysis from 1-iode-4-methylbenzene (13j, $\left.54.5 \mathrm{mg}, 0.25 \mathrm{mmol}\right)$.

${ }^{1} \mathrm{H}$ and ${ }^{13} \mathrm{C}$ NMR were in agreement with the reference ${ }^{6} .{ }^{1} \mathbf{H} \mathbf{~ N M R}\left(\mathbf{C D C l}_{\mathbf{3}}, \mathbf{5 0 0} \mathbf{M H z}\right): 7.41-7.32(\mathrm{~m}, 2 \mathrm{H}), 7.15(\mathrm{~d}$, $2 \mathrm{H}, J=7.2 \mathrm{~Hz}), 2.34(\mathrm{~s}, 3 \mathrm{H}), 1.57-1.49(\mathrm{~m}, 6 \mathrm{H}), 1.33(\mathrm{tq}, 6 \mathrm{H}, J=7.4,7.1 \mathrm{~Hz}), 1.08-0.99(\mathrm{~m}, 6 \mathrm{H}), 0.88(\mathrm{t}, 9 \mathrm{H}, J$ $=7.3 \mathrm{~Hz}) .{ }^{13} \mathbf{C} \mathbf{~ N M R}\left(\mathbf{C D C l}_{\mathbf{3}}, \mathbf{1 2 5} \mathbf{~ M H z}\right): 138.0,137.8,136.6,129.0,29.2,27.5,21.5,13.8,9.7 . \mathbf{R}_{\mathbf{f}}=0.81$ (hexane). EI-MS: $m / z$ : $[\mathrm{M}-\mathrm{Bu}]^{+}$calcd for $\mathrm{C}_{15} \mathrm{H}_{25} \mathrm{Sn} 325.1$; Found 325.0.

\section{(4-benzylphenyl)tributylstannane (12k)}

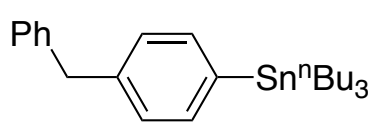

Using the Typical Procedure B, the titled compound was obtained as a colorless oil in $37 \%$ NMR yield determined by ${ }^{1} \mathrm{H}$ NMR analysis (isolated yield: $21 \%, 23.7 \mathrm{mg}$ ) from 1-benzyl-4-fluorobenzene (11k, $46.6 \mathrm{mg}, 0.25 \mathrm{mmol})$. Using the Typical Procedure B (fluoride source: KF and 18-crown-6, solvent: THF), the titled compound was obtained in 70\% NMR yield determined by ${ }^{1} \mathrm{H}$ NMR analysis from 1-benzyl-4-iodobenzene (13k, $\left.73.5 \mathrm{mg}, 0.25 \mathrm{mmol}\right)$.

${ }^{1} \mathbf{H} \mathbf{N M R}\left(\mathbf{C D C l}_{3}, \mathbf{5 0 0} \mathbf{M H z}\right):$ 7.41-7.33 (m, 2H), $7.29(\mathrm{dd}, 2 \mathrm{H}, J=7.6,7.6 \mathrm{~Hz}), 7.22-7.19(\mathrm{~m}, 3 \mathrm{H}), 7.15(\mathrm{~d}, 2 \mathrm{H}$, $J=7.6 \mathrm{~Hz}), 3.96(\mathrm{~s}, 2 \mathrm{H}), 1.57-1.49(\mathrm{~m}, 6 \mathrm{H}), 1.32(\mathrm{tq}, 6 \mathrm{H}, J=7.4,7.3 \mathrm{~Hz}), 1.07-0.98(\mathrm{~m}, 6 \mathrm{H}), 0.88(\mathrm{t}, 9 \mathrm{H}, J=7.3$ $\mathrm{Hz}) .{ }^{13} \mathbf{C} \mathbf{N M R}\left(\mathbf{C D C l}_{3}, \mathbf{1 2 5} \mathbf{M H z}\right): 141.3,140.9,139.1,137.6,136.8,129.1,128.7,126.2,42.1,29.2,27.5,13.8$, 9.7. $\mathbf{R}_{\mathbf{f}}=0.34$ (hexane). HRMS (APCI): $m / z:[\mathrm{M}-\mathrm{Bu}]^{+}$calcd for $\mathrm{C}_{21} \mathrm{H}_{29} \mathrm{Sn}$ 401.1286; Found 401.1283. ATR-FTIR (neat) v: 2955, 2924, 2850, 1591, 1454, 1376, 1069, 864, 739, $695 \mathrm{~cm}^{-1}$. 
Tributyl(mesityl)stannane (12l)

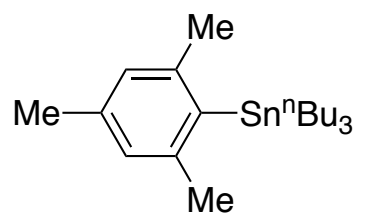

Using the Typical Procedure B, the titled compound was obtained as a colorless oil in $22 \%(22.4 \mathrm{mg})$ isolated yield from 2-fluoro-1,3,5-trimethylbenzene (111, $34.5 \mathrm{mg}, 0.25$ mmol). Using the Typical Procedure B (fluoride source: KF and 18-crown-6, solvent: THF), the titled compound was obtained in $28 \%$ NMR yield determined by ${ }^{1} \mathrm{H}$ NMR analysis from 2-iodo-1,3,5-trimethylbenzene (131, $73.5 \mathrm{mg}, 0.25 \mathrm{mmol})$.

${ }^{1} \mathrm{H}$ and ${ }^{13} \mathrm{C}$ NMR were in agreement with the reference ${ }^{8} .{ }^{1} \mathbf{H} \mathbf{~ N M R}\left(\mathbf{C D C l}_{3}, \mathbf{5 0 0} \mathbf{M H z}\right): 6.83(\mathrm{~d}, 2 \mathrm{H}, J=7.0 \mathrm{~Hz})$, $2.36(\mathrm{~s}, 6 \mathrm{H}), 2.25(\mathrm{~s}, 3 \mathrm{H}), 1.53-1.46(\mathrm{~m}, 6 \mathrm{H}), 1.33(\mathrm{tq}, 6 \mathrm{H}, J=7.4,7.2 \mathrm{~Hz}), 1.10-1.05(\mathrm{~m}, 6 \mathrm{H}), 0.88(\mathrm{t}, 9 \mathrm{H}, J=7.3$

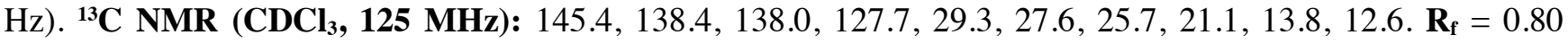
(hexane). EI-MS: $m / z$ : [M-Bu] ${ }^{+}$calcd for $\mathrm{C}_{17} \mathrm{H}_{29} \mathrm{Sn} 353.1$; Found 353.1 .

\section{Tributyl(naphthalen-1-yl)stannane (12m)}

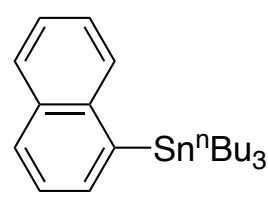

Using the Typical Procedure B, the titled compound was obtained as a colorless oil in 54\% NMR yield determined by ${ }^{1} \mathrm{H}$ NMR analysis (isolated yield: $31 \%, 32.2 \mathrm{mg}$ ) from 1fluoronaphthalene $(\mathbf{1 1 m}, 36.5 \mathrm{mg}, 0.25 \mathrm{mmol})$.

${ }^{1} \mathrm{H}$ and ${ }^{13} \mathrm{C}$ NMR were in agreement with the reference ${ }^{6} .{ }^{1} \mathbf{H} \mathbf{~ N M R}\left(\mathbf{C D C l}_{3}, \mathbf{5 0 0} \mathbf{M H z}\right): 7.88$ $7.75(\mathrm{~m}, 3 \mathrm{H}), 7.69-7.57(\mathrm{~m}, 1 \mathrm{H}), 7.52-7.41(\mathrm{~m}, 3 \mathrm{H}), 1.61-1.53(\mathrm{~m}, 6 \mathrm{H}), 1.35$ (tq, $6 \mathrm{H}, J=7.3$, $7.3 \mathrm{~Hz}), 1.26-1.15(\mathrm{~m}, 6 \mathrm{H}), 0.88(\mathrm{t}, 9 \mathrm{H}, J=7.3 \mathrm{~Hz}) .{ }^{13} \mathbf{C} \mathbf{N M R}\left(\mathbf{C D C l}_{3}, \mathbf{1 2 5} \mathbf{~ M H z}\right): 143.1,139.6,135.2,133.8$, $130.3,129.0,128.6,125.8,125.5,29.3,27.5,13.8,10.5 . \mathbf{R}_{\mathbf{f}}=0.50$ (hexane). EI-MS: $m / z:[\mathrm{M}-\mathrm{Bu}]^{+}$calcd for $\mathrm{C}_{18} \mathrm{H}_{25} \mathrm{Sn} 361.1$; Found 361.1.

\section{2-(tributylstannyl)pyridine (12n)}

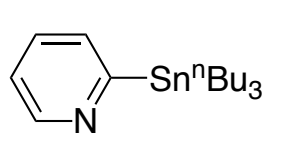

Using the Typical Procedure B, the titled compound was obtained as a colorless oil in $77 \%$ NMR yield determined by ${ }^{1} \mathrm{H}$ NMR analysis (isolated yield: $37 \%, 33.6 \mathrm{mg}$ ) from 2fluoropyridine (11n, $24.3 \mathrm{mg}, 0.25 \mathrm{mmol}$ ). Using the Typical Procedure B (fluoride source: KF and 18-crown-6, solvent: THF), the titled compound was obtained in 75\% NMR yield determined by ${ }^{1} \mathrm{H}$ NMR analysis from 2-iodepyridine (13n, $51.3 \mathrm{mg}, 0.25 \mathrm{mmol})$.

${ }^{1} \mathrm{H}$ and ${ }^{13} \mathrm{C}$ NMR were in agreement with the reference ${ }^{7} .{ }^{1} \mathbf{H} \mathbf{~ N M R}\left(\mathbf{C D C l}_{3}, \mathbf{5 0 0} \mathbf{M H z}\right): 8.73(\mathrm{~d}, 1 \mathrm{H}, J=4.9 \mathrm{~Hz})$, $7.48(\mathrm{dd}, 1 \mathrm{H}, J=7.4,7.4 \mathrm{~Hz}), 7.43-7.36(\mathrm{~m}, 1 \mathrm{H}), 7.13-7.08(\mathrm{~m}, 1 \mathrm{H}), 1.60-1.52(\mathrm{~m}, 6 \mathrm{H}), 1.33(\mathrm{tq}, 6 \mathrm{H}, J=7.4,7.2$ $\mathrm{Hz}), 1.15-1.09(\mathrm{~m}, 6 \mathrm{H}), 0.88(\mathrm{t}, 9 \mathrm{H}, J=7.3 \mathrm{~Hz}) .{ }^{13} \mathbf{C} \mathbf{N M R}\left(\mathbf{C D C l}_{3}, \mathbf{1 2 5} \mathbf{M H z}\right):$ 174.2, 150.7, 133.4, 132.5, 122.1, 29.2, 27.5, 13.8, 9.9. $\mathbf{R}_{\mathbf{f}}=0.24$ (hexane:AcOEt $=20: 1$ ). EI-MS: $m / z:[\mathrm{M}-\mathrm{Bu}]^{+}$calcd for $\mathrm{C}_{13} \mathrm{H}_{22} \mathrm{NSn} 312.1$; Found 312.0 .

\section{3-methyl-2-(tributylstannyl)pyridine (120)}

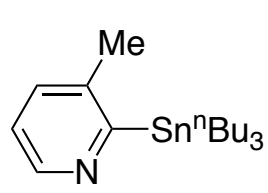

Using the Typical Procedure B, the titled compound was obtained as a colorless oil in $84 \%$ NMR yield determined by ${ }^{1} \mathrm{H}$ NMR analysis (isolated yield: 24\%, $22.9 \mathrm{mg}$ ) from 2-fluoro-3methylpyridine (11o, $27.8 \mathrm{mg}, 0.25 \mathrm{mmol})$.

${ }^{1} \mathbf{H}$ NMR $\left(\mathbf{C D C l}_{3}, \mathbf{5 0 0} \mathbf{M H z}\right): 8.54(\mathrm{dd}, 1 \mathrm{H}, J=4.8,1.1 \mathrm{~Hz}), 7.36-7.29(\mathrm{~m}, 1 \mathrm{H})$, 7.04-6.99 $(\mathrm{m}, 1 \mathrm{H}), 2.36(\mathrm{~s}, 3 \mathrm{H}), 1.58-1.50(\mathrm{~m}, 6 \mathrm{H}), 1.33(\mathrm{tq}, 6 \mathrm{H}, J=7.4,7.1 \mathrm{~Hz}), 1.18-1.10(\mathrm{~m}, 6 \mathrm{H}), 0.87(\mathrm{t}, 9 \mathrm{H}, J=7.3$ $\mathrm{Hz}) .{ }^{13} \mathbf{C}$ NMR $\left(\mathbf{C D C l}_{3}, \mathbf{1 2 5} \mathbf{M H z}\right): 174.5,147.4,140.7,134.2,121.7,29.3,27.5,21.9,13.8,10.6 . \mathbf{R}_{\mathbf{f}}=0.67$ (hexane : $\mathrm{AcOEt}=4: 1)$. HRMS (ESI): $\mathrm{m} / z:[\mathrm{M}+\mathrm{H}]^{+} \mathrm{Calcd}$ for $\mathrm{C}_{18} \mathrm{H}_{34} \mathrm{NSn} 384.1708$; Found 384.1709. ATR-FTIR (neat) v: 2955, 2922, 2852, 1568, 1463, 1290, 1070, 960, 783, $666 \mathrm{~cm}^{-1}$. 


\section{4-methyl-2-(tributylstannyl)pyridine (12p)}

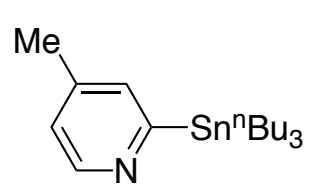

Using the Typical Procedure B, the titled compound was obtained as a yellow oil in $69 \%$ yield NMR yield determined by ${ }^{1} \mathrm{H}$ NMR analysis (isolated yield: 25\%, $24.1 \mathrm{mg}$ ) from 2fluoro-4-methylpyridine (11p, $27.8 \mathrm{mg}, 0.25 \mathrm{mmol})$.

${ }^{1} \mathrm{H}$ and ${ }^{13} \mathrm{C}$ NMR were in agreement with the reference ${ }^{9} .{ }^{1} \mathbf{H} \mathbf{~ N M R}\left(\mathbf{C D C l}_{\mathbf{3}}, \mathbf{5 0 0} \mathbf{M H z}\right): 8.58$ $(\mathrm{d}, 1 \mathrm{H}, J=5.0 \mathrm{~Hz}), 7.21(\mathrm{dd}, 1 \mathrm{H}, J=9.4,9.4 \mathrm{~Hz}), 6.95-6.92(\mathrm{~m}, 1 \mathrm{H}), 2.29(\mathrm{~s}, 3 \mathrm{H}), 1.60-1.52(\mathrm{~m}, 6 \mathrm{H}), 1.33(\mathrm{tq}$, 6H, $J=7.4,7.3 \mathrm{~Hz}), 1.17-1.04(\mathrm{~m}, 6 \mathrm{H}), 0.88(\mathrm{t}, 9 \mathrm{H}, J=7.2 \mathrm{~Hz}) .{ }^{13} \mathbf{C} \mathbf{N M R}\left(\mathbf{C D C l}_{3}, \mathbf{1 2 5} \mathbf{M H z}\right): 173.3,150.3$, 144.1, 133.5, 123.2, 29.2, 27.5, 21.1, 13.8, 9.9. $\mathbf{R}_{\mathbf{f}}=0.55$ (hexane:AcOEt = 4:1). EI-MS: $m / z:[\mathrm{M}-\mathrm{Bu}]^{+}$calcd for $\mathrm{C}_{14} \mathrm{H}_{24} \mathrm{NSn} 326.1$; Found 326.0.

\section{Methyl 3-(tributylstannyl)thiophene-2-carboxylate (12q)}

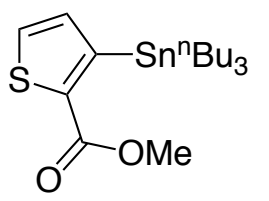

Using the Typical Procedure B, the titled compound was obtained as a yellow oil in $31 \%$ NMR yield determined by ${ }^{1} \mathrm{H}$ NMR analysis (isolated yield: $22 \%, 24.1 \mathrm{mg}$ ) from methyl 3fluorothiophene-2-carboxylate (11q, $40.0 \mathrm{mg}, 0.25 \mathrm{mmol})$.

${ }^{1} \mathbf{H}$ NMR $\left(\mathbf{C D C l}_{3}, \mathbf{5 0 0} \mathbf{M H z}\right):$ 7.61-7.58 (m, 1H), 7.19-7.15 (m, 1H), $3.88(\mathrm{~s}, 3 \mathrm{H}), 1.54-1.46$ $(\mathrm{m}, 6 \mathrm{H}), 1.30(\mathrm{tq}, 6 \mathrm{H}, J=7.4,7.1 \mathrm{~Hz}), 1.13-1.07(\mathrm{~m}, 6 \mathrm{H}), 0.87(\mathrm{t}, 9 \mathrm{H}, J=7.4 \mathrm{~Hz}) .{ }^{13} \mathbf{C ~ N M R}$ $\left(\mathbf{C D C l}_{3}, \mathbf{1 2 5}\right.$ MHz): $164.0,151.5,137.9,135.0,131.8,52.1,29.3,27.5,13.8,11.0 . \mathbf{R}_{\mathbf{f}}=0.36($ hexane:AcOEt $=$ 40:1). HRMS (APCI): $m / z$ : [M-Bu] ${ }^{+}$calcd for $\mathrm{C}_{14} \mathrm{H}_{23} \mathrm{O}_{2} \mathrm{SSn}$ 375.0435; Found 375.0412. ATR-FTIR (neat) v: $2954,2921,2843,1704,1392,1253,1072,874,768,603 \mathrm{~cm}^{-1}$.

\section{4-(tributylstannyl)benzoic acid (12r)}

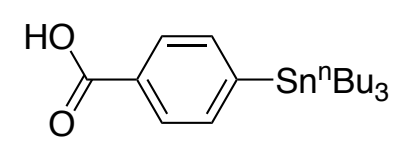

Using the Typical Procedure B (fluoride source: KF and 18-crown-6, solvent: THF), the titled compound was obtained as a yellow oil in $82 \%$ NMR yield determined by ${ }^{1} \mathrm{H}$ NMR analysis (isolated yield: 31\%, $40.8 \mathrm{mg}$ ) from 4-iodobenzoic acid (13r, $61.5 \mathrm{mg}$, $0.25 \mathrm{mmol}) .23 \mathrm{wt} \%$ of tributylhydroxystannane $\left(\mathrm{Bu}_{3} \mathrm{Sn}-\mathrm{OH}\right)$ is including in an isolated product.

${ }^{1} \mathbf{H}$ NMR (CDCl 3 , 500 MHz): 8.04-7.94 (m, 2H), 7.64-7.48 (m, 2H), 1.57-1.49 (m, 6H), 1.37-1.31 (m, 6H), 1.15$1.02(\mathrm{~m}, 6 \mathrm{H}), 0.88(\mathrm{t}, 9 \mathrm{H}, J=7.3 \mathrm{~Hz}) .{ }^{13} \mathbf{C ~ N M R}\left(\mathbf{C D C l}_{3}, \mathbf{1 2 5} \mathbf{M H z}\right): 172.1,151.2,136.6,129.1,29.2,27.5,13.8$, 9.8. $\mathbf{R}_{\mathbf{f}}=0.43$ (hexane:AcOEt = 4:1). HRMS (ESI): $\mathrm{m} / \mathrm{z}$ : $[\mathrm{M}-\mathrm{H}]^{-}$calcd for $\mathrm{C}_{19} \mathrm{H}_{31} \mathrm{O}_{2} \mathrm{Sn} 411.1352$; Found 411.1352 . ATR-FTIR (neat) v: 2955, 2923, 2853, 1691, 1552, 1288, 1062, 844, 758, $683 \mathrm{~cm}^{-1}$.

\section{2-(4-ethylpiperazin-1-yl)-4-(4-(tributylstannyl)phenyl)-5,6,7,8,9,10-hexahydrocycloocta[b]pyridine (12s)}

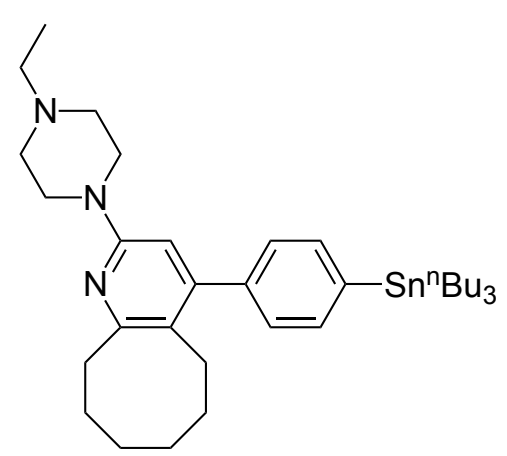

Using the Typical Procedure B, the titled compound was obtained as a colorless oil in $31 \%{ }^{1} \mathrm{H}$ NMR analysis (isolated yield: $8 \%, 13.1 \mathrm{mg}$ ) from blonanserin (11s, $26.7 \mathrm{mg}, 0.073 \mathrm{mmol})$.

${ }^{1} \mathbf{H}$ NMR $\left(\mathbf{C D C l}_{\mathbf{3}}, \mathbf{5 0 0} \mathbf{M H z}\right):$ 7.52-7.41 (m, 2H), 7.22-7.18 (m, 2H), $6.34(\mathrm{~s}$, $1 \mathrm{H}), 3.55(\mathrm{~m}, 4 \mathrm{H}), 2.93-2.84(\mathrm{~m}, 2 \mathrm{H}), 2.62-2.56(\mathrm{~m}, 6 \mathrm{H}), 2.53-2.43(\mathrm{~m}, 2 \mathrm{H})$, $1.83-1.75(\mathrm{~m}, 3 \mathrm{H}), 1.60-1.53(\mathrm{~m}, 8 \mathrm{H}), 1.34-1.33(\mathrm{~m}, 8 \mathrm{H}), 1.14(\mathrm{t}, 2 \mathrm{H}, J=7.2$ $\mathrm{Hz}), 1.12-1.03(\mathrm{~m}, 6 \mathrm{H}), 0.94-0.88(\mathrm{~m}, 11 \mathrm{H}) .{ }^{13} \mathbf{C} \mathbf{~ N M R}\left(\mathbf{C D C l}_{3}, \mathbf{1 2 5} \mathbf{~ M H z}\right)$ : $159.9,157.4,151.7,141.2,140.9,136.1,128.1,123.2,106.2,52.8,52.6,45.6$, $35.7,31.8,30.8,29.3,27.5,26.8,26.7,26.0,13.8,12.0,9.8 . \mathbf{R}_{\mathbf{f}}=0.10$ (hexane:AcOEt = 4:1). HRMS (ESI): $\mathrm{m} / z:[\mathrm{M}+\mathrm{H}]^{+}$Calcd for $\mathrm{C}_{35} \mathrm{H}_{58} \mathrm{~N}_{3} \mathrm{Sn}$ 640.3647; Found 640.3666. ATR-FTIR (neat) v: 2954, 2922, 2850, 1583, 1448, 1378, 1245, 998, 818, $755 \mathrm{~cm}^{-1}$. 


\subsection{Sequential Stannylation/Stille Coupling Processes (Scheme 2)}

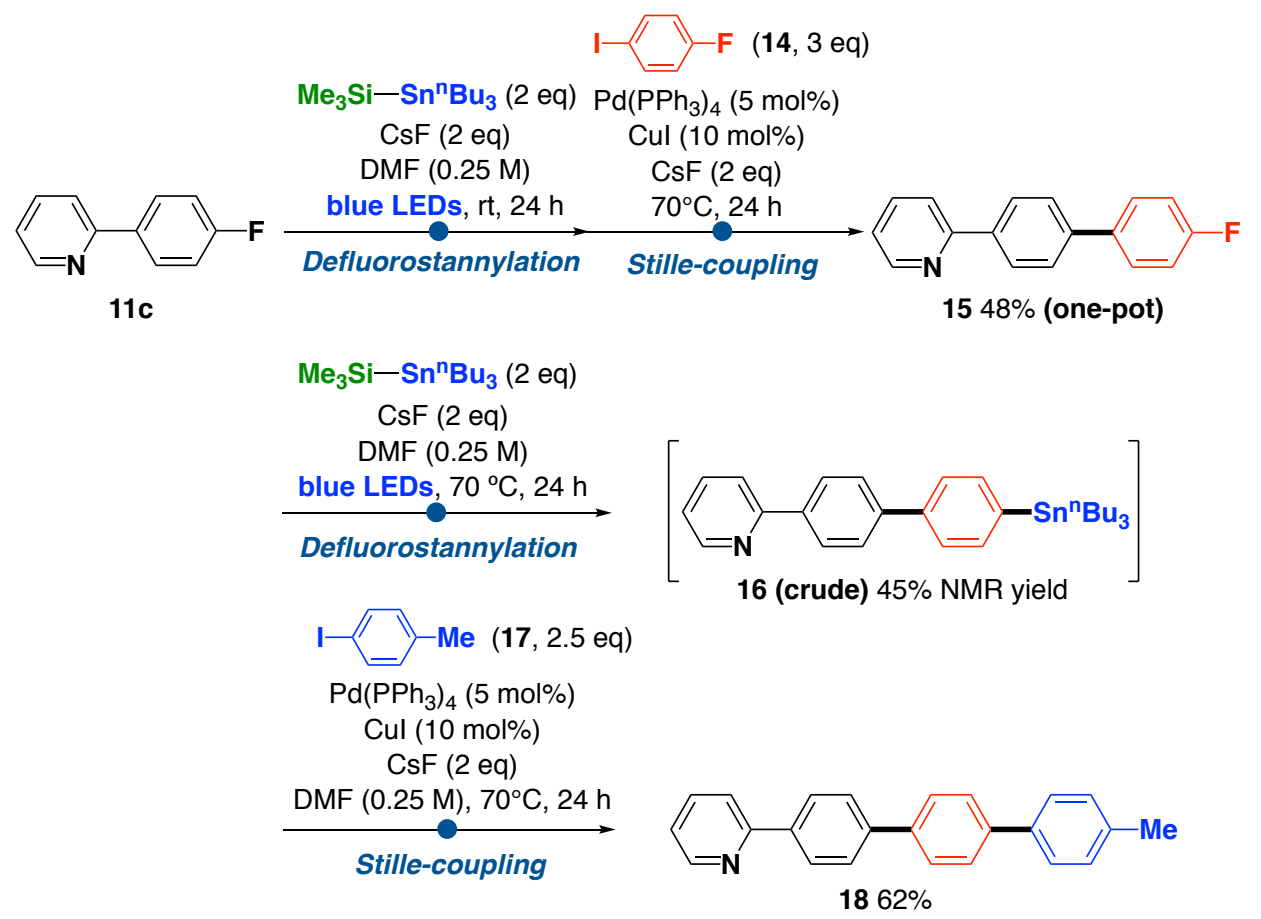

CsF (76.0 mg, $0.50 \mathrm{mmol}$ ) and 2-(4-fluorophenyl)pyridine (11c, $43.3 \mathrm{mg}, 0.25 \mathrm{mmol})$ were charged in a dried tube at argon glovebox and dissolved in dry DMF $(1 \mathrm{~mL})$. To the mixture was successively added trimethyl(tributylstannyl)silane $(2,182 \mathrm{mg}, 0.50 \mathrm{mmol})$ at room temperature. After the reaction tube was sealed and irradiated with a blue LED lamp, the solution was stirred for $24 \mathrm{~h}$ at room temperature. Then, after $\mathrm{Pd}\left(\mathrm{PPh}_{3}\right)_{4}$ (14.4 mg, $0.0125 \mathrm{mmol})$, CuI (4.8 mg, $0.0025 \mathrm{mmol}), \mathrm{CsF}$ (76.0 mg, $0.50 \mathrm{mmol}), 1$-fluoro-4-iodobenzene (14, $167 \mathrm{mg}, 0.75 \mathrm{mmol})$, and DMF $(2.5 \mathrm{~mL})$ were successively added to the mixture, the resulting mixture was heated to $70{ }^{\circ} \mathrm{C}$ and stirred for $24 \mathrm{~h}$. The reaction was quenched by $\mathrm{H}_{2} \mathrm{O}(1 \mathrm{~mL})$, and then extracted with AcOEt $(3 \mathrm{~mL}$ $\times 3)$. The AcOEt layers were combined and dried over $\mathrm{MgSO}_{4}$, and the solvent was removed under reduced pressure. The residue was purified by silica gel column chromatography using hexane:AcOEt $=9: 1$ as eluent to give 2-(4'fluoro-[1,1'-biphenyl]-4-yl)pyridine $(\mathbf{1 5}, 25.0 \mathrm{mg}, 2$ steps $40 \%$ yield from 11c) as white solid (48\% NMR yield determined by ${ }^{1} \mathrm{H}$ NMR analysis from 11c).

Then, CsF (44.4 mg, $0.29 \mathrm{mmol}$ ) and 2-(4'-fluoro-[1,1'-biphenyl]-4-yl)pyridine (15,24.3 mg, $0.098 \mathrm{mmol})$ were charged in a dried tube at argon glovebox and dissolved in dry DMF $(0.5 \mathrm{~mL})$. To the mixture was successively added trimethyl(tributylstannyl)silane $(2,106 \mathrm{mg}, 0.29 \mathrm{mmol})$ at room temperature. After the reaction tube was sealed and irradiated with a blue LED lamp, the solution was heated to $70{ }^{\circ} \mathrm{C}$ and stirred for $24 \mathrm{~h}$. The reaction was quenched by $\mathrm{H}_{2} \mathrm{O}(1 \mathrm{~mL})$, and then extracted with AcOEt $(3 \mathrm{~mL} \times 3)$. The AcOEt layers were combined and dried over $\mathrm{MgSO}_{4}$, and the solvent was removed under reduced pressure to give crude of 2-(4'-(tributylstannyl)[1,1'-biphenyl]-4-yl)pyridine (16, 60\% NMR yield determined by ${ }^{1} \mathrm{H}$ NMR analysis from 15) as a colorless oil.

Finally, $\mathrm{Pd}\left(\mathrm{PPh}_{3}\right)_{4}(6.2 \mathrm{mg}, 0.0054 \mathrm{mmol}), \mathrm{CuI}(2.1 \mathrm{mg}, 0.010 \mathrm{mmol}), \mathrm{CsF}$ (33.1 mg, $\left.0.22 \mathrm{mmol}\right)$, 4-iodotoluene $(\mathbf{1 7}, 59.5 \mathrm{mg}, 0.27 \mathrm{mmol})$, and DMF $(1.5 \mathrm{~mL})$ were successively added to a portion of the crude mixture 16 (including $0.037 \mathrm{mmol}$ of 16), and the resulting mixture was heated to $70{ }^{\circ} \mathrm{C}$ and stirred for $24 \mathrm{~h}$. The reaction was quenched by $\mathrm{H}_{2} \mathrm{O}(1 \mathrm{~mL})$, and then extracted with AcOEt $(3 \mathrm{~mL} \times 3)$. The AcOEt layers were combined and dried over $\mathrm{MgSO}_{4}$, and the solvent was removed under reduced pressure. The residue was purified by silica gel column chromatography using hexane:AcOEt =9:1 as eluent to give 2-(4"-methyl-[1,1':4',1"-terphenyl]-4-yl)pyridine (18, $7.3 \mathrm{mg}, 0.023 \mathrm{mmol}, 62 \%$ yield) as a white solid. 


\section{2-(4'-fluoro-[1,1'-biphenyl]-4-yl)pyridine (15)}

${ }^{1} \mathbf{H}$ NMR $\left(\mathbf{C D C l}_{3}, \mathbf{5 0 0} \mathbf{M H z}\right): 8.72(\mathrm{dd}, 1 \mathrm{H}, J=4.8,1.3 \mathrm{~Hz}), 8.08(\mathrm{dd}, 2 \mathrm{H}, J=8.5,2.0 \mathrm{~Hz}), 7.79-7.76(\mathrm{~m}, 2 \mathrm{H})$, $7.66(\mathrm{dd}, 2 \mathrm{H}, J=8.6,2.0 \mathrm{~Hz}), 7.64-7.59(\mathrm{~m}, 2 \mathrm{H}), 7.26-7.23(\mathrm{~m}, 1 \mathrm{H}), 7.15(\mathrm{dd}, 2 \mathrm{H}, J=8.7,8.7 \mathrm{~Hz}) .{ }^{13} \mathbf{C} \mathbf{N M R}$ $\left(\mathbf{C D C l}_{3}, \mathbf{1 2 5} \mathrm{MHz}\right): 162.8(\mathrm{~d}, J=371.3 \mathrm{~Hz}), 157.1,149.9,140.9,138.5,136.92,136.87,136.85,128.9$ (d, $J=8.1$ $\mathrm{Hz}), 127.5(\mathrm{~d}, J=4.3 \mathrm{~Hz}), 122.3,120.6,115.8(\mathrm{~d}, J=21.1 \mathrm{~Hz}) .{ }^{19} \mathbf{F} \mathbf{N M R}\left(\mathbf{C D C l}_{3}, \mathbf{3 7 6} \mathbf{M H z}\right):-115.4 . \mathbf{R}_{\mathbf{f}}=0.22$ (hexane:AcOEt =10:1). HRMS (ESI): $\mathrm{m} / z$ : $[\mathrm{M}+\mathrm{H}]^{+}$Calcd for $\mathrm{C}_{17} \mathrm{H}_{13} \mathrm{FN} 250.1027$; Found 250.1026. ATR-FTIR (neat) v: $3051,3010,1584,1462,1297,1153,1012,823,775,685 \mathrm{~cm}^{-1}$. mp: $172.5-177.7^{\circ} \mathrm{C}$.

\section{2-(4'-(tributylstannyl)-[1,1'-biphenyl]-4-yl)pyridine (16)}

${ }^{1} \mathbf{H}$ NMR $\left(\mathbf{C D C l}_{3}, \mathbf{5 0 0} \mathbf{M H z}\right): 8.71(\mathrm{dd}, 1 \mathrm{H}, J=4.7,1.4 \mathrm{~Hz}), 8.08(\mathrm{dd}, 2 \mathrm{H}, J=8.5,1.9 \mathrm{~Hz}), 7.80-7.74(\mathrm{~m}, 2 \mathrm{H})$, $7.73(\mathrm{~d}, 2 \mathrm{H}, J=8.4 \mathrm{~Hz}), 7.65-7.53(\mathrm{~m}, 2 \mathrm{H}), 7.25-7.22(\mathrm{~m}, 1 \mathrm{H}), 1.63-1.54(\mathrm{~m}, 6 \mathrm{H}), 1.36(\mathrm{tq}, 6 \mathrm{H}, J=7.4,7.3 \mathrm{~Hz})$, 1.17-1.04 (m, 6H), $0.91(\mathrm{t}, 9 \mathrm{H}, J=7.3 \mathrm{~Hz}) .{ }^{13} \mathbf{C} \mathbf{N M R}\left(\mathbf{C D C l}_{3}, \mathbf{1 2 5}_{\mathbf{M H z}}\right):$ 157.3, 149.9, 142.0, 141.5, 140.2, 138.4, 137.1, 136.9, 127.5, 127.4, 126.7, 122.2, 120.6, 29.3, 27.5, 13.8, 9.8. $\mathbf{R}_{\mathbf{f}}=0.34$ (hexane:AcOEt =10:1). HRMS (ESI): $m / z:[\mathrm{M}+\mathrm{H}]^{+}$Calcd for $\mathrm{C}_{29} \mathrm{H}_{40} \mathrm{NSn} 522.2177$; Found 522.2190. ATR-FTIR (neat) v: 2955, 2923, $2851,1586,1465,1294,1152,1075,1003,777 \mathrm{~cm}^{-1}$.

\section{2-(4"'-methyl-[1,1':4',1"'-terphenyl]-4-yl)pyridine (18)}

${ }^{1} \mathbf{H}$ NMR $\left(\mathbf{C D C l}_{3}, \mathbf{5 0 0} \mathbf{M H z}\right): 8.72(\mathrm{dd}, 1 \mathrm{H}, J=4.6,1.3 \mathrm{~Hz}), 8.10(\mathrm{dd}, 2 \mathrm{H}, J=8.5,1.8 \mathrm{~Hz}), 7.81-7.75(\mathrm{~m}, 4 \mathrm{H})$, 7.75-7.67 (m, 4H), $7.56(\mathrm{~d}, 2 \mathrm{H}, J=7.2 \mathrm{~Hz}), 7.28(\mathrm{~d}, 2 \mathrm{H}, J=7.8 \mathrm{~Hz}), 7.26-7.23(\mathrm{~m}, 1 \mathrm{H}), 2.42(\mathrm{~s}, 3 \mathrm{H}) .{ }^{13} \mathbf{C} \mathbf{N M R}$ $\left(\mathbf{C D C l}_{3}, \mathbf{1 2 5} \mathbf{M H z}\right):$ 157.2, 149.9, 141.4, 140.5, 139.3, 138.4, 137.9, 137.4, 136.9, 129.7, 127.6, 127.50, 127.48, 127.45, 127.0, 122.3, 120.6, 21.3. $\mathbf{R}_{\mathbf{f}}=0.14$ (hexane:AcOEt $\left.=10: 1\right)$. HRMS (ESI): $m / z:[\mathrm{M}+\mathrm{H}]^{+} \mathrm{Calcd}_{\text {for }} \mathrm{C}_{24} \mathrm{H}_{20} \mathrm{~N}$ 322.1590; Found 322.1594. ATR-FTIR (neat) v: 3019, 2962, 2913, 1585, 1464, 1260, 1215, 1093, 1011, 753, $667 \mathrm{~cm}^{-1}$. mp: $256.6-270.9^{\circ} \mathrm{C}$.

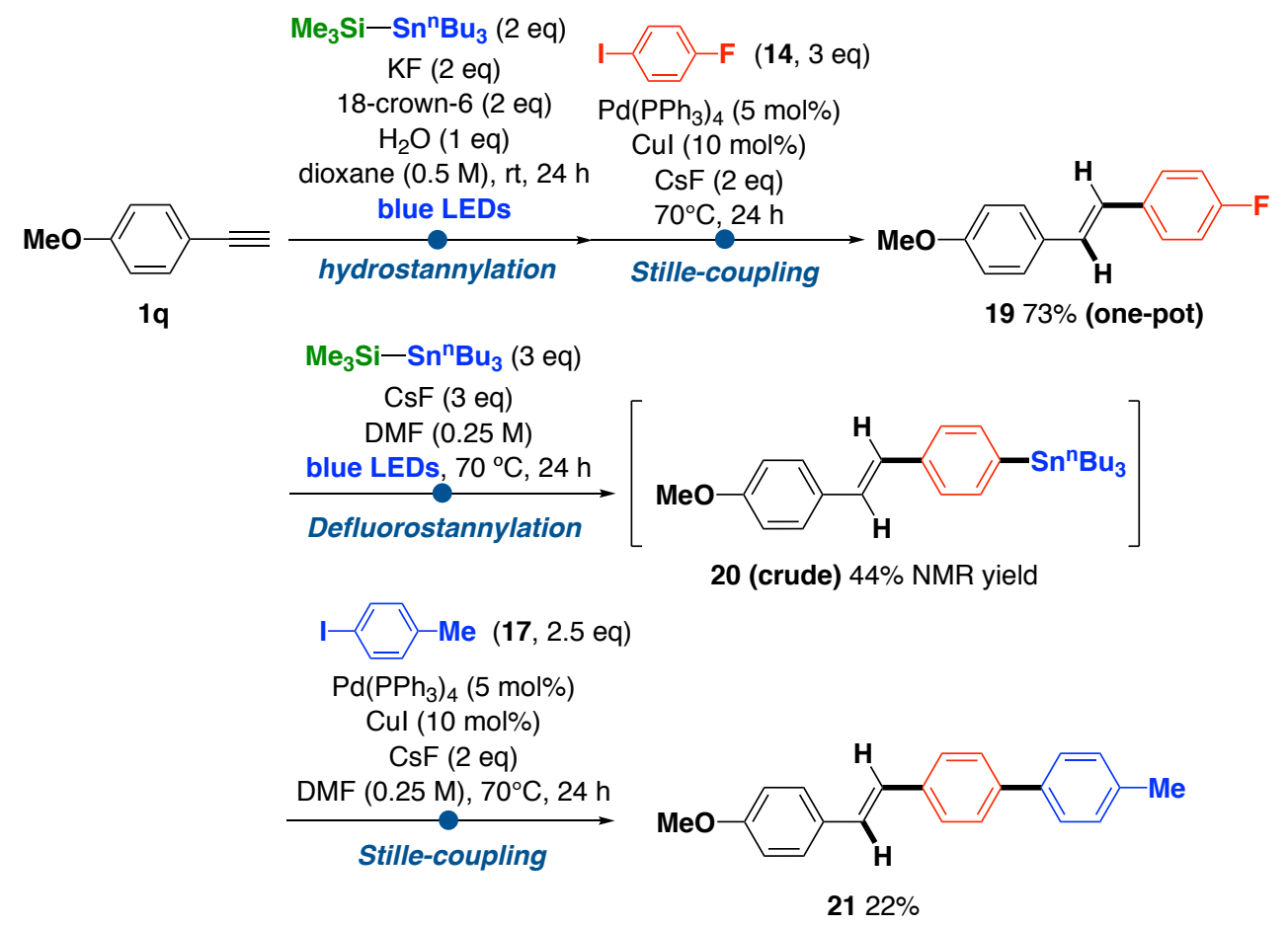

KF (29.1 mg, $0.50 \mathrm{mmol}), 18$-crown-6 (132 mg, $0.50 \mathrm{mmol})$ and 1-ethynyl-4-methoxybenzene (1q, $33 \mathrm{mg}, 0.25$ mmol) were charged in a dried tube at argon glovebox and dissolved in dry DMF (1 mL). To the mixture was successively added trimethyl(tributylstannyl)silane $(2,182 \mathrm{mg}, 0.50 \mathrm{mmol})$ and $\mathrm{H}_{2} \mathrm{O}(4.5 \mathrm{mg}, 0.25 \mathrm{mmol})$ at room 
temperature. After the reaction tube was sealed and irradiated with a blue LED lamp, the solution was stirred for $24 \mathrm{~h}$ at room temperature. Then, after $\mathrm{Pd}\left(\mathrm{PPh}_{3}\right)_{4}(14.4 \mathrm{mg}, 0.0125 \mathrm{mmol}), \mathrm{CuI}$ (4.8 mg, $\left.0.0025 \mathrm{mmol}\right), \mathrm{CsF}$ (76.0 $\mathrm{mg}, 0.50 \mathrm{mmol}), 1$-fluoro-4-iodobenzene $(\mathbf{1 4}, 167 \mathrm{mg}, 0.75 \mathrm{mmol})$, and DMF $(2.5 \mathrm{~mL})$ were successively added to the mixture, the resulting mixture was heated to $70{ }^{\circ} \mathrm{C}$ and stirred for $24 \mathrm{~h}$. The reaction was quenched by $\mathrm{H}_{2} \mathrm{O}$ $(1 \mathrm{~mL})$, and then extracted with AcOEt $(3 \mathrm{~mL} \times 3)$. The AcOEt layers were combined and dried over $\mathrm{MgSO}_{4}$, and the solvent was removed under reduced pressure. The residue was purified by silica gel column chromatography using hexane:AcOEt =19:1 as eluent to give (E)-1-fluoro-4-(4-methoxystyryl)benzene (19, $36.8 \mathrm{mg}, 2$ steps $64 \%$ yield from 1q) as a white solid (73\% NMR yield determined by ${ }^{1} \mathrm{H}$ NMR analysis from 1q).

Then, $\mathrm{CsF}$ (73.5 mg, $0.48 \mathrm{mmol}$ ) and (E)-1-fluoro-4-(4-methoxystyryl)benzene (19, 36.8 $\mathrm{mg}, 0.16 \mathrm{mmol})$ were charged in a dried tube at argon glovebox and dissolved in dry DMF $(0.6 \mathrm{~mL})$. To the mixture was successively added trimethyl(tributylstannyl)silane $(2,176 \mathrm{mg}, 0.48 \mathrm{mmol})$ at room temperature. After the reaction tube was sealed and irradiated with a blue LED lamp, the solution was heated to $70{ }^{\circ} \mathrm{C}$ and stirred for $24 \mathrm{~h}$. The reaction was quenched by $\mathrm{H}_{2} \mathrm{O}(1 \mathrm{~mL})$, and then extracted with AcOEt $(3 \mathrm{~mL} \times 3)$. The AcOEt layers were combined and dried over $\mathrm{MgSO}_{4}$, and the solvent was removed under reduced pressure to give crude of $(E)$-tributyl(4-(4methoxystyryl)phenyl)stannane (20,44\% NMR yield determined by ${ }^{1} \mathrm{H}$ NMR analysis from 19) as a colorless oil.

Finally, $\mathrm{Pd}\left(\mathrm{PPh}_{3}\right)_{4}(7.4 \mathrm{mg}, 0.0064 \mathrm{mmol}), \mathrm{CuI}(2.4 \mathrm{mg}, 0.013 \mathrm{mmol}), \mathrm{CsF}$ (39.0 mg, $\left.0.26 \mathrm{mmol}\right)$, 4-iodotoluene $(\mathbf{1 7}, 84.0 \mathrm{mg}, 0.39 \mathrm{mmol})$, and DMF $(0.5 \mathrm{~mL})$ were successively added to a portion of the crude mixture 20 (including $0.0217 \mathrm{mmol}$ of 20), and the resulting mixture was heated to $70{ }^{\circ} \mathrm{C}$ and stirred for $24 \mathrm{~h}$. The reaction was quenched by $\mathrm{H}_{2} \mathrm{O}(1 \mathrm{~mL})$, and then extracted with AcOEt $(3 \mathrm{~mL} \times 3)$. The AcOEt layers were combined and dried over $\mathrm{MgSO}_{4}$, and the solvent was removed under reduced pressure. The residue was purified by silica gel column chromatography using hexane:AcOEt =19:1 as eluent to give (E)-4-(4-methoxystyryl)-4'-methyl-1,1'biphenyl (21, $1.4 \mathrm{mg}, 0.0047 \mathrm{mmol}, 22 \%$ yield) as a white solid.

\section{(E)-1-fluoro-4-(4-methoxystyryl)benzene (19)}

${ }^{1} \mathrm{H}$ and ${ }^{13} \mathrm{C}$ NMR were in agreement with the reference ${ }^{10}{ }^{1} \mathbf{H} \mathbf{~ N M R}\left(\mathbf{C D C l}_{3}, \mathbf{5 0 0} \mathbf{~} \mathbf{M H z}\right): 7.56-7.51(\mathrm{~m}, 4 \mathrm{H}), 7.14$ $(\mathrm{dd}, 2 \mathrm{H}, J=8.8,8.8 \mathrm{~Hz}), 7.10-7.02(\mathrm{~m}, 2 \mathrm{H}), 7.00(\mathrm{~d}, 2 \mathrm{H}, J=8.8 \mathrm{~Hz}), 3.93(\mathrm{~s}, 3 \mathrm{H}) .{ }^{13} \mathbf{C} \mathbf{~ N M R}(\mathbf{C D C l}, \mathbf{1 2 5} \mathbf{M H z})$ : $162.2(J=370.5 \mathrm{~Hz}), 159.5,134.0(\mathrm{~d}, J=3.8 \mathrm{~Hz}), 130.1,128.2(\mathrm{~d}, J=2.5 \mathrm{~Hz}), 127.84,127.79,125.6,115.7(\mathrm{~d}, J$ $=21.5 \mathrm{~Hz}), 114.3,55.5 .{ }^{19} \mathbf{F} \mathbf{N M R}\left(\mathbf{C D C l}_{3}, \mathbf{3 7 6} \mathbf{M H z}\right):-114.9 . \mathbf{R}_{\mathbf{f}}=0.48$ (hexane:AcOEt =20:1). EI-MS: $\mathrm{m} / \mathrm{z}:[\mathrm{M}]$ calcd for $\mathrm{C}_{15} \mathrm{H}_{13} \mathrm{FO} 228.1$; Found 228.1.

(E)-tributyl(4-(4-methoxystyryl)phenyl)stannane (20)

${ }^{1} \mathbf{H}$ NMR $\left(\mathbf{C D C l}_{3}, \mathbf{5 0 0} \mathbf{M H z}\right)$ : 7.50-7.36 (m, 6H), 7.11-6.92 (m, 2H), 6.90 (d, 2H, J = 8.7 Hz), 3.83 (s, 3H), 1.59$1.50(\mathrm{~m}, 6 \mathrm{H}), 1.34(\mathrm{tq}, 6 \mathrm{H}, J=7.4,7.1 \mathrm{~Hz}), 1.12-1.00(\mathrm{~m}, 6 \mathrm{H}), 0.89(\mathrm{t}, 9 \mathrm{H}, J=7.3 \mathrm{~Hz}) .{ }^{13} \mathbf{C} \mathbf{N M R}\left(\mathbf{C D C l}_{3}, \mathbf{1 2 5}\right.$ MHz): $159.4,141.4,137.4,136.9,130.4,128.1,127.8,127.0,125.9,114.3,55.5,29.3,27.5,13.8,9.8 . \mathbf{R}_{\mathbf{f}}=0.39$ (hexane:AcOEt =20:1). HRMS (ESI): $\mathrm{m} / z:[\mathrm{M}+\mathrm{H}]^{+}$calcd for $\mathrm{C}_{27} \mathrm{H}_{41} \mathrm{OSn} 501.2174$; Found 501.2171. ATR-FTIR (neat) v: 2954, 2924, 2851, 1510, 1463, 1249, 1173, 961, $823 \mathrm{~cm}^{-1}$.

\section{(E)-4-(4-methoxystyryl)-4'-methyl-1,1'-biphenyl (21)}

${ }^{1} \mathrm{H}$ and ${ }^{13} \mathrm{C}$ NMR were in agreement with the reference ${ }^{11} .{ }^{1} \mathbf{H} \mathbf{~ N M R}\left(\mathbf{C D C l}_{3}, \mathbf{5 0 0} \mathbf{M H z}\right): 7.49(\mathrm{~d}, 2 \mathrm{H}, J=8.2 \mathrm{~Hz})$, $7.46(\mathrm{~d}, 2 \mathrm{H}, J=8.3 \mathrm{~Hz}), 7.32(\mathrm{~d}, 2 \mathrm{H}, J=8.0 \mathrm{~Hz}), 7.25-7.22(\mathrm{~m}, 4 \mathrm{H}), 6.78(\mathrm{~d}, 2 \mathrm{H}, J=8.8 \mathrm{~Hz}), 6.58-6.50(\mathrm{~m}, 2 \mathrm{H})$, 3.80 (s, 3H), 2.39 (s, 3H). ${ }^{13} \mathbf{C}$ NMR ( $\left.\mathbf{C D C l}_{3}, \mathbf{1 2 5} \mathbf{~ M H z}\right):$ 158.7, 139.7, 138.0, 137.2, 136.4, 130.3, 130.0, 129.9, 129.6, 129.4, 128.6, 126.9, 126.8, 113.8, 55.4, 21.2. $\mathbf{R}_{\mathbf{f}}=0.37$ (hexane:AcOEt =20:1). EI-MS: $\mathrm{m} / \mathrm{z}:[\mathrm{M}]$ calcd for $\mathrm{C}_{22} \mathrm{H}_{20} \mathrm{O} 300.2$; Found 300.1 . 


\section{Computational Details}

All calculations were carried with the Gaussian 16 program package. ${ }^{13}$ The hybrid density functional method based on UB3LYP ${ }^{14}$ with a standard $6-31+\mathrm{g}^{*}$ basis set (LANL2DZ basis set for Sn) was used for geometry optimizations. Excitation wavelengths and oscillator strengths were obtained at the density functional level using time-dependent perturbation theory (TD-DFT) approach. Geometry optimization and vibrational analysis were performed at the same level. All stationary points were optimized without any symmetry assumptions, and characterized by normal coordinate analysis at the same level of theory (number of imaginary frequencies, NIMAG, 0 for minima and 1 for TSs). The intrinsic reaction coordinate (IRC) method was used to track minimum energy paths from transition structures to the corresponding local minima. ${ }^{15}$

Table S1. The Gibbs-free energies and imaginary frequencies.

\begin{tabular}{|lll|}
\hline & $G$ (hartree) & imaginary frequency $\left(\mathrm{cm}^{-1}\right)$ \\
\hline $\mathbf{A}\left(\mathrm{SnMe}_{3}\right.$ anion$)$ & -123.066523 & \\
\hline${ }^{3} \mathbf{A}\left(\mathrm{SnMe}_{3}\right.$ anion $)$ & -122.950247 & \\
\hline propyne & -116.629606 & -209.90 \\
\hline $\mathbf{B}$ (biradical complex) & -239.616242 & -105.56 \\
\hline INT (propyne + Sn anion) & -239.69117 & \\
\hline TS1 & -239.641324 & -70.51 \\
\hline TS2 & -239.647250 \\
\hline C-1 & -239.668448 \\
\hline C-2 & -239.671193 \\
\hline${ }^{3}[$ TS1 $]$ & -239.605339 \\
\hline${ }^{3}[\mathbf{C}-1]$ & -239.616974 & \\
\hline${ }^{3}[\mathbf{C}-2]$ & -239.54042 & \\
\hline
\end{tabular}


$\mathrm{A}$ (SnMe 3 anion)

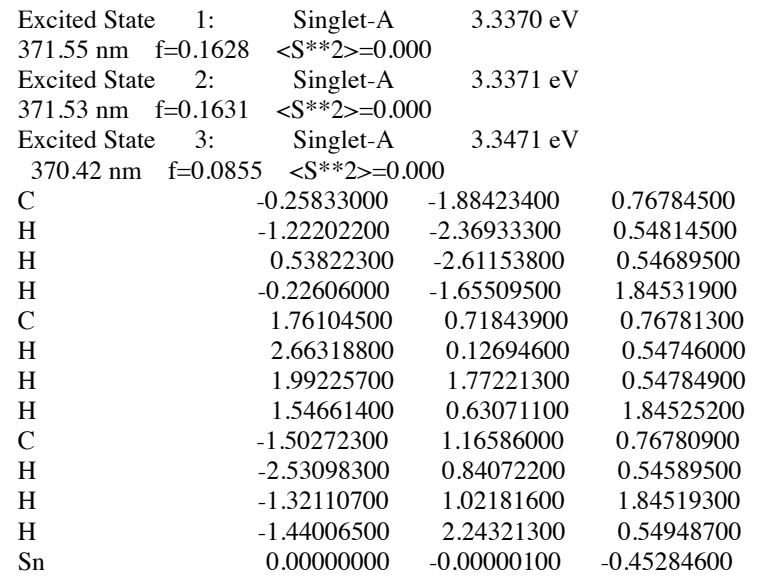

\section{${ }^{3} A^{*}$ (SnMe 3 anion)}

$\begin{array}{lrrr}\mathrm{C} & -0.16239900 & 2.05180300 & 0.42880400 \\ \mathrm{H} & 0.67694100 & 2.63849600 & 0.03712700 \\ \mathrm{H} & -1.08439100 & 2.49858100 & 0.03837200 \\ \mathrm{H} & -0.16460000 & 2.08907200 & 1.52580100 \\ \mathrm{C} & -1.69647400 & -1.16585000 & 0.42912300 \\ \mathrm{H} & -2.62393700 & -0.73022200 & 0.03899300 \\ \mathrm{H} & -1.62455100 & -2.18732100 & 0.03733700 \\ \mathrm{H} & -1.72669000 & -1.18775400 & 1.52613000 \\ \mathrm{C} & 1.85859100 & -0.88490100 & 0.42920700 \\ \mathrm{H} & 2.70603200 & -0.30658500 & 0.04244500 \\ \mathrm{H} & 1.89017300 & -0.90553400 & 1.52619200 \\ \mathrm{H} & 1.94991600 & -1.90348700 & 0.03400800 \\ \mathrm{Sn} & 0.00005600 & -0.00023100 & -0.25058400\end{array}$

\section{Propyne}

C

$\mathrm{H}$
$\mathrm{H}$

$\mathrm{C}$

C

$\mathrm{H}$

B

$\begin{array}{lrrr}\mathrm{C} & 5.96519200 & -0.00130400 & 0.19127700 \\ \mathrm{H} & 6.30051100 & -0.36677500 & 1.17068400 \\ \mathrm{H} & 6.37406000 & 1.00864600 & 0.05686400 \\ \mathrm{H} & 6.41586800 & -0.64588100 & -0.57466100 \\ \mathrm{C} & 4.50589900 & -0.00137800 & 0.09479000 \\ \mathrm{C} & 3.29715500 & -0.00141800 & 0.01397300 \\ \mathrm{H} & 2.22070900 & -0.00141400 & -0.05599500 \\ \mathrm{C} & -1.18049600 & -2.35742100 & -0.40072700 \\ \mathrm{H} & -0.74939600 & -2.64648000 & -1.36623000 \\ \mathrm{H} & -0.56631200 & -2.75543400 & 0.41875500 \\ \mathrm{H} & -2.20890500 & -2.73727100 & -0.32111100 \\ \mathrm{C} & -2.15960100 & 0.00298200 & 1.79889100 \\ \mathrm{H} & -1.39232900 & 0.00467000 & 2.58718900 \\ \mathrm{H} & -2.78663800 & 0.89490300 & 1.93948600 \\ \mathrm{H} & -2.78584800 & -0.88901300 & 1.94239100 \\ \mathrm{C} & -1.17150800 & 2.35716600 & -0.40291800 \\ \mathrm{H} & -0.73907500 & 2.64368900 & -1.36858000 \\ \mathrm{H} & -2.19849300 & 2.74097100 & -0.32389500 \\ \mathrm{H} & -0.55604200 & 2.75363000 & 0.41635700 \\ \mathrm{Sn} & -1.25735900 & 0.00008000 & -0.24585900 \\ & & & \\ \mathrm{INT} & & & \\ \mathrm{C} & & & -0.00045100 \\ \mathrm{H} & -6.11786200 & -0.00044800 & -0.56079100 \\ \mathrm{H} & -6.51783500 & -0.85585600 & -0.46103300 \\ \mathrm{H} & -6.51825900 & 0.91235300 & -0.00045200 \\ \mathrm{C} & -6.51794000 & -0.05815800 & 1.0204500\end{array}$

$\begin{array}{lll}-1.42803100 & 0.00000400 & 0.00000600\end{array}$ $\begin{array}{lll}1.24330300 & -0.00001300 & -0.00001100\end{array}$

$\begin{array}{lll}1.63741000 & 0.56117200 & -0.85571800\end{array}$

$\begin{array}{lll}1.63744600 & -1.02163900 & -0.05809000\end{array}$

$\begin{array}{lll}1.63743900 & 0.46057000 & 0.91377600\end{array}$

$\begin{array}{lll}-0.21813500 & -0.00001100 & 0.00002300\end{array}$

$\begin{array}{lll}-2.49511200 & 0.00001200 & -0.00007800\end{array}$

C

$\mathrm{H}$
$\mathrm{H}$

$\mathrm{H}$

$\mathrm{H}$
$\mathrm{C}$

C

$\mathrm{H}$
$\mathrm{C}$

$\mathrm{H}$

$\mathrm{H}$

H

C

$\mathrm{H}$

H

C

$\mathrm{H}$
$\mathrm{H}$

$\mathrm{H}$

$\mathrm{Sn}$

C-1

$\mathrm{C}$
$\mathrm{H}$
$\mathrm{H}$
$\mathrm{H}$
$\mathrm{C}$
$\mathrm{C}$
$\mathrm{H}$
$\mathrm{C}$
$\mathrm{H}$
$\mathrm{H}$
$\mathrm{H}$
$\mathrm{C}$
$\mathrm{H}$

$\begin{array}{rrr}-3.44294700 & 0.00055800 & -0.00059900 \\ -2.36127800 & 0.00099900 & -0.00095300 \\ 2.14040100 & -0.95578200 & 1.65382300 \\ 1.91219800 & -0.49193900 & 2.62544100 \\ 1.91267300 & -2.02936900 & 1.73652500 \\ 3.22059100 & -0.84730200 & 1.46643900 \\ 2.14831200 & -0.95390200 & -1.65125800 \\ 1.92156300 & -2.02753900 & -1.73605500 \\ 1.92413400 & -0.48928400 & -2.62344800 \\ 3.22756400 & -0.84484100 & -1.45899800 \\ 2.14409400 & 1.90901900 & 0.00293700 \\ 1.91526800 & 2.51787400 & 0.89073400 \\ 3.22401600 & 1.69108500 & 0.00497100 \\ 1.91889600 & 2.51891300 & -0.88509300 \\ 0.94873400 & 0.00012700 & -0.00084600\end{array}$

4.73368400

4.25144900

4.25133200

2.58661500

1.77906900

1.71802000

$-0.47179900$

0.57870100

$-0.92439000$

$-0.99892200$

$-2.85752400$

$-3.19048300$

$-3.19062900$

$-3.35205400$

$-0.47187500$

0.57859100

$-0.99940300$

$-0.92407700$

$-0.59986000$

0.00018100

$-0.87713300$

0.87689900

$-0.00003300$

0.00014000

0.00034200

1.69731900

1.91039700

2.60067900

1.46811500

0.00010400

0.88907100

$-0.88935300$

0.00062400

$-1.69758400$

$-1.91043700$

$-1.46864800$

$-2.60099900$

0.00001400

0.23362100

$-0.61702200$

0.85319100

0.85350700

$-0.16627100$

$-1.13862400$

$-2.21898800$

1.19081400

1.42507000

0.75764900

2.12879600

$-0.46371400$

$-1.01854700$

$-1.01767900$

0.52065300

1.19048500

1.42508500

2.12830200

0.75702700

$-0.22109800$

$\begin{array}{ccc}-5.61398300 & -0.00001500 & -0.00156000 \\ -6.03805800 & -0.06041300 & 1.01518400 \\ -6.03375400 & -0.85128800 & -0.56404200 \\ -6.03384200 & 0.91181400 & -0.45932600 \\ -4.14342800 & -0.00017100 & 0.00248900 \\ -2.89924000 & -0.00048600 & 0.00592700 \\ -0.92425400 & -0.00022100 & 0.00472300 \\ 1.83615200 & -0.21606300 & 1.95975000 \\ 1.54562000 & -1.16180800 & 2.43496700 \\ 2.93082100 & -0.20142100 & 1.86711700 \\ 1.53888200 & 0.59872700 & 2.63236200 \\ 1.82763500 & -1.59062900 & -1.17067000 \\ 1.53401900 & -2.57992100 & -0.79681300 \\ 1.52835900 & -1.53165800 & -2.22499600 \\ 2.92269700 & -1.51708600 & -1.12086700 \\ 1.82716200 & 1.80776900 & -0.79580800 \\ 1.53083300 & 1.97777900 & -1.83885700 \\ 1.53039600 & 2.69235100 & -0.21780000 \\ 2.92225400 & 1.72672500 & -0.75986400 \\ 0.88080500 & -0.00012000 & 0.00054900\end{array}$

$\begin{array}{rrr}-3.89808100 & -0.10616500 & 0.00014800 \\ -4.03362600 & -1.21886700 & 0.00047900 \\ -4.43427900 & 0.29253600 & -0.87664700 \\ -4.43427300 & 0.29307100 & 0.87669300 \\ -2.44911800 & 0.35188300 & -0.00003000 \\ -1.52058000 & -0.63743000 & 0.00020900 \\ -1.77961800 & -1.72533800 & 0.00056900 \\ 1.05633500 & 1.16961000 & -1.72251900 \\ 1.04212600 & 0.56457600 & -2.63844200 \\ 0.33484400 & 1.98563300 & -1.84577000 \\ 2.06080400 & 1.59902900 & -1.60685200 \\ 1.05715000 & 1.16973900 & 1.72222300 \\ 0.33701000 & 1.98708900 & 1.84461400\end{array}$




$\begin{array}{lrrr}\mathrm{H} & 1.04139300 & 0.56535200 & 2.63854400 \\ \mathrm{H} & 2.06241700 & 1.59735800 & 1.60679200 \\ \mathrm{C} & 1.92087400 & -1.69123400 & -0.00017900 \\ \mathrm{H} & 1.78793000 & -2.32575000 & -0.88669100 \\ \mathrm{H} & 2.95622100 & -1.32358000 & -0.00013000 \\ \mathrm{H} & 1.78797500 & -2.32614900 & 0.88605000 \\ \mathrm{Sn} & 0.48543200 & -0.03006800 & 0.00003400\end{array}$

\section{C-2}

C

$\mathrm{H}$

H

$\mathrm{H}$

C

$\mathrm{H}$

C

$\mathrm{H}$

H

$\mathrm{H}$
$\mathrm{C}$

H

$\mathrm{H}$
$\mathrm{H}$

H

H

H

$\mathrm{H}$
$\mathrm{Sn}$

\section{${ }^{3}[\mathrm{TS} 1]$}

$\mathrm{H}$

H

H

C

C

$\mathrm{H}$

H

$\mathrm{H}$

$\mathrm{C}$

H

$\mathrm{H}$

C

$\mathrm{H}$
$\mathrm{H}$

H

Sn

${ }^{3}[\mathrm{C}-1]$

C

H

$\mathrm{H}$
$\mathrm{H}$

$\mathrm{H}$
$\mathrm{C}$

C

$\mathrm{H}$

$\mathrm{H}$

$\mathrm{H}$

$\mathrm{H}$

I

$\mathrm{H}$

$\mathrm{C}$
$\mathrm{H}$
$\mathrm{H}$

$\mathrm{Sn}$
$-4.42664400$

$-4.83090500$

$-4.86044600$

$-4.83102400$

$-2.95969100$

$-1.72754900$

0.50812200

0.53430100

$-0.05514200$

1.53125000

0.01394300

2.91533900

3.27938700

3.27943700

3.38321500

0.53439900

$-0.05511100$

0.01413100

1.53135900
0.63741700

$0.00000400 \quad-0.08513600$

$-0.88092700 \quad 0.43788400$

$-0.00548100 \quad-1.09746900$

$0.88647900 \quad 0.42834500$

$-0.00005900-0.11312400$

$0.00007900 \quad-0.14522600$

$0.00002000 \quad-1.95279800$

$-1.89365600 \quad 0.88947600$

-2.61984300 0.31577900

$-2.30894900 \quad 1.08572400$

$-1.73558800 \quad 1.84317700$

$-0.00006600-0.30547900$

$-0.88913400 \quad-0.84610300$

$0.88897900 \quad-0.84610700$

$\begin{array}{ll}-0.00007700 & 0.69274500\end{array}$

$1.89367700 \quad 0.88946200$

$2.61983700 \quad 0.31580000$

$1.73562300 \quad 1.84321400$

$2.30899000 \quad 1.08561000$

0.00000400

$-0.20171300$

4.42171700

5.12358000

4.66112800

4.66016300

2.99661300

2.26318500

2.58505300

$-1.20698600$

$-1.10456100$

$-0.56669100$

$-2.25495400$

$-1.20701700$

$-0.56577100$

$-1.10606400$

$-2.25452600$

$-2.34506100$

$-2.31659400$

$-3.29404300$

$-2.31712800$

$-0.61568600$
0.63063600

$-0.23445700$

1.24484900

1.24272500

0.18952800

$-0.87620200$

$-1.93940200$

$-1.12788200$

$-0.58375900$

$-2.01626600$

$-1.44654900$

$-1.11560000$

$-2.00289200$

$-0.56420700$

$-1.43623800$

1.57762900

2.21742200

1.01877400

2.22371400

0.13215300
$-0.00155400$

$-0.00089900$

0.87958600

$-0.88443200$

$-0.00026600$

0.00138500

0.00248600

$-1.70750100$

$-2.65526500$

$-1.75338700$

$-1.60285700$

1.71577100

1.76890700

2.65948300

1.61259800

$-0.00587000$

$-0.89762600$

$-0.00418900$

0.88135700

$-0.00035100$

$$
\begin{array}{cc}
-0.22843700 & 0.29270600 \\
0.66037100 & 0.85837300 \\
-1.10424800 & 0.94680700 \\
-0.35579400 & -0.57041200 \\
-0.08483100 & -0.09724900 \\
0.66708400 & 0.12496800 \\
1.63877500 & 0.65057100 \\
-0.77416900 & 1.09938500 \\
-0.08520200 & 1.88421200 \\
-1.76402600 & 1.54613600 \\
-0.85017100 & 0.31976200 \\
-1.39969000 & -1.53200900 \\
-2.42680900 & -1.20058000 \\
-1.11134100 & -2.24256900 \\
-1.39822700 & -2.05069700 \\
1.83159400 & -0.76438300 \\
2.71807000 & -0.14456600 \\
1.73975700 & -0.90020500 \\
2.00152300 & -1.74603000 \\
0.00536000 & 0.15817400
\end{array}
$$

${ }^{3}$ [C-2]

$\begin{array}{ccc}-4.21228900 & 0.04763800 & -0.01868200 \\ -4.77082500 & -0.70460800 & -0.59637700 \\ -4.30189700 & 1.01797000 & -0.55521500 \\ -4.70605000 & 0.15698900 & 0.95881200 \\ -2.76364300 & -0.29724000 & 0.10912600 \\ -1.68815400 & 0.37308300 & -0.33548300 \\ 0.68452900 & 0.12221200 & -1.98630700 \\ 0.81708500 & 1.93135300 & 0.85334500 \\ 0.08431700 & 2.65228700 & 0.47592100 \\ 0.60820200 & 1.76258500 & 1.91853500 \\ 1.83171400 & 2.33866900 & 0.75891800 \\ 0.11919800 & -1.85782900 & 0.81380000 \\ -0.94867400 & -2.03550300 & 0.96088600 \\ 0.55293500 & -2.67168700 & 0.21944400 \\ 0.62705300 & -1.82375700 & 1.78619100 \\ 2.81100100 & -0.46546300 & -0.07790100 \\ 3.41318800 & 0.31475300 & -0.56717600 \\ 3.13632200 & -0.54634700 & 0.97016400 \\ 3.01892900 & -1.42307400 & -0.57811500 \\ 0.60542100 & 0.04900500 & -0.23661800\end{array}$




\section{References}

(1) Eisch, J. J. \& Galle, E. J. J. Organomet. Chem. 1988, 341, 293.

(2) Cheng, L. \& Mankad, P. N. J. Am. Chem. Soc. 2019, 141, 3710.

(3) Darwish, A.; Lang, A.; Kim, T.; Chong, J. M. Org. Lett. 2008, 10, 861.

(4) Miura, K.; Wang, D.; Matsumoto, Y.; Hosomi, A. Org. Lett. 2004. 7, 503.

(5) Du, Z.; Zhou, W.; Bai, L.; Wang, F.; Wang, J. Synlett 2011, 3, 369.

(6) Yue, H.; Zhu, C.; Rueping, M. Org. Lett. 2018, 20, 385.

(7) Wang, D.; Wen, X.: Xiong, C.; Zhao, J.; Ding, C.; Meng, Q.; Zhou, H.; Wang, C.; Uchiyama, M.; Lu, X.; Zhang, A. iScience 2019, 15, 307.

(8) Furuya, T.; Strom, E. A.; Ritter, T. J. Am. Chem. Soc. 2009, 131, 1662.

(9) Zhang, G.; Zong, R.; Tseng, H.; Thummel, P. R. Inorganic Chemistry 2008, 47, 991.

(10) Kalkhambkar, R.G. \& Laali, K.K. Tetrahedron Letters 2011, 52, 1733.

(11) Lim, D. S. W. \& Anderson, E. A. Org. Lett. 2011, 13, 4806.

(12) Donohoe, J. T.; Basutto, A. J.; Bower, F. J.; Rathi, A. Org. Lett, 2011, 13, 1036.

(13) Gaussian 16, Revision A.03, Frisch, M. J.; Trucks, G. W.; Schlegel, H. B.; Scuseria, G. E.; Robb, M. A.; Cheeseman, J. R.; Scalmani, G.; Barone, V.; Petersson, G. A.; Nakatsuji, H.; Li, X.; Caricato, M.; Marenich, A. V.; Bloino, J.; Janesko, B. G.; Gomperts, R.; Mennucci, B.; Hratchian, H. P.; Ortiz, J. V.; Izmaylov, A. F.; Sonnenberg, J. L.; Williams-Young, D.; Ding, F.; Lipparini, F.; Egidi, F.; Goings, J.; Peng, B.; Petrone, A.; Henderson, T.; Ranasinghe, D.; Zakrzewski, V. G.; Gao, J.; Rega, N.; Zheng, G.; Liang, W.; Hada, M.; Ehara, M.; Toyota, K.; Fukuda, R.; Hasegawa, J.; Ishida, M.; Nakajima, T.; Honda, Y.; Kitao, O.; Nakai, H.; Vreven, T.; Throssell, K.; Montgomery, J. A.; Jr., Peralta, J. E.; Ogliaro, F.; Bearpark, M. J.; Heyd, J. J.; Brothers, E. N.; Kudin, K. N.; Staroverov, V. N.; Keith, T. A.; Kobayashi, R.; Normand, J.; Raghavachari, K.; Rendell, A. P.; Burant, J. C.; Iyengar, S. S.; Tomasi, J.; Cossi, M.; Millam, J. M.; Klene, M.; Adamo, C.; Cammi, R.; Ochterski, J. W.; Martin, R. L.; Morokuma, K.; Farkas, O.; Foresman, J. B.; Fox, D. J. Gaussian, Inc., Wallingford CT, 2016.

(14) (a) Beche, A. D. Phys. Rev. 1988, A38, 3098-3100. (b) Beche, A. D. J. Chem. Phys. 1993, 98, 1372-1377. (c) Beche, A. D. J. Chem. Phys. 1993, 98, 5648-5652. (d) Lee, C; Yang, W.; Parr, R. G. Phys. Rev. 1988, B37, 785-788.

(15) (a) Fukui, K. Acc. Chem. Res. 1981, 14, 363-368. (b) Ishida, K.; Morokuma, K.; Komornicki, A. J. Chem. Phys. 1977, 66, 2153-2156. (c) Gonzalez, C.; Schlegel, H. B. J. Chem. Phys. 1989, 90, 2154-2161. (d) Schlegel, H. B.; Gonzalez, C. J. Phys. Chem. 1990, 94, 5523-5527. 


\section{Copies of NMR Spectra}

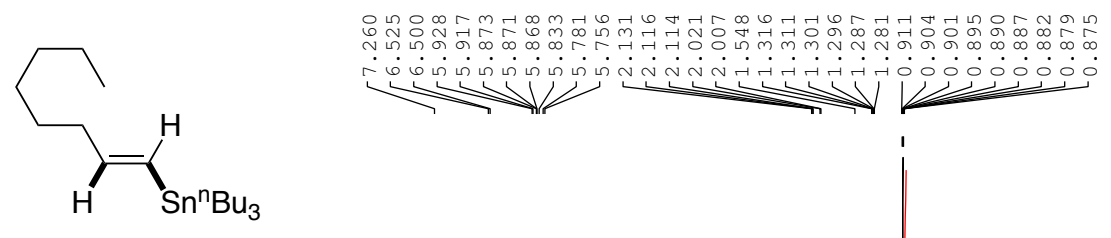

* isomer
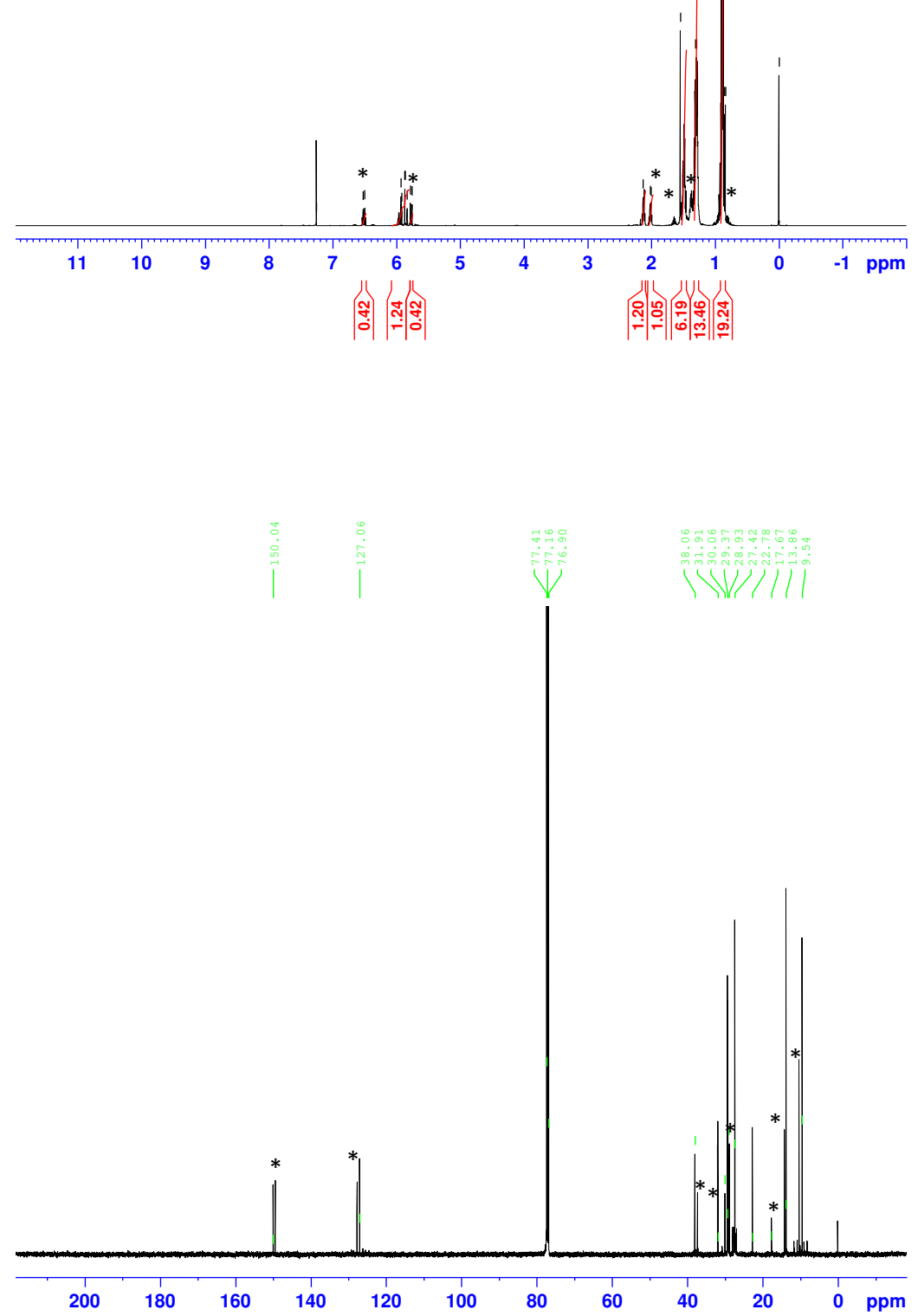

S-24 

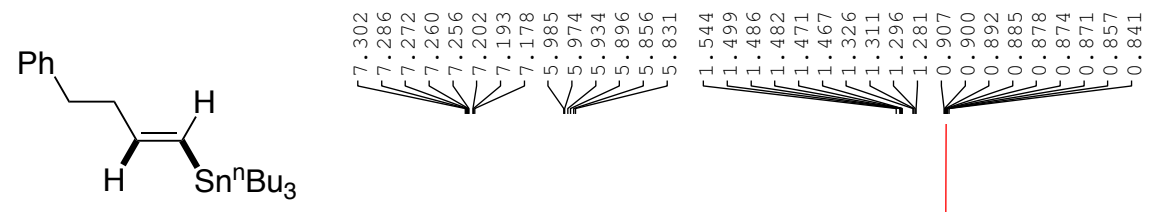

* isomer
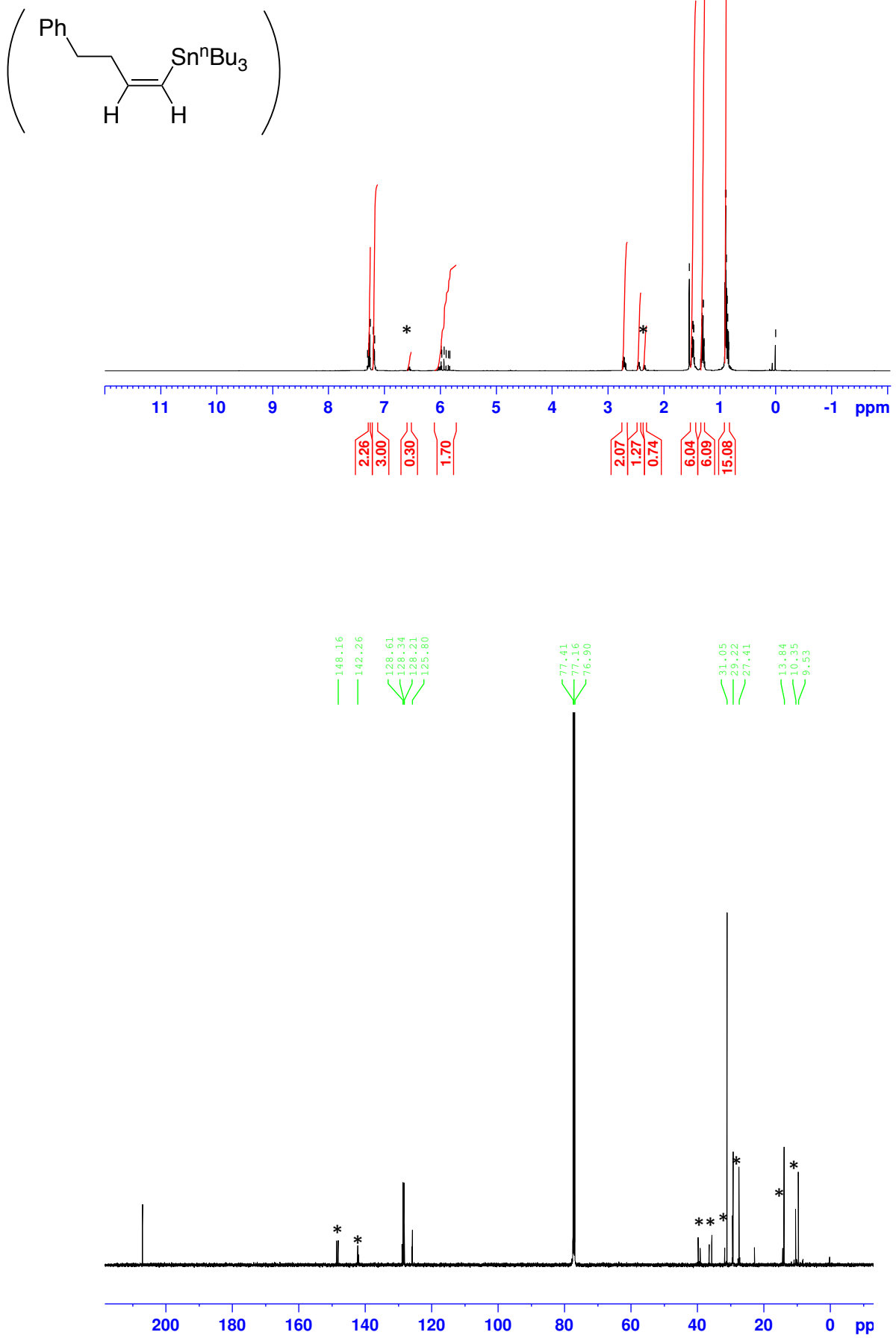

S-25 


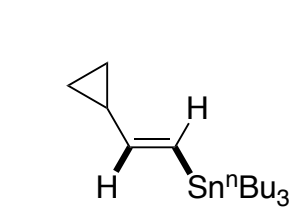

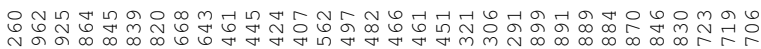

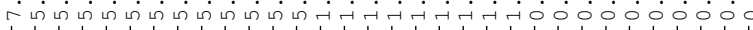

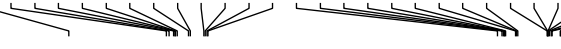

* isomer

$\left(\searrow_{\mathrm{H}} \mathrm{L}_{\mathrm{H}}^{\mathrm{Sn}^{\mathrm{n}} \mathrm{Bu}_{3}}\right)$

In $^{*^{* *}}$
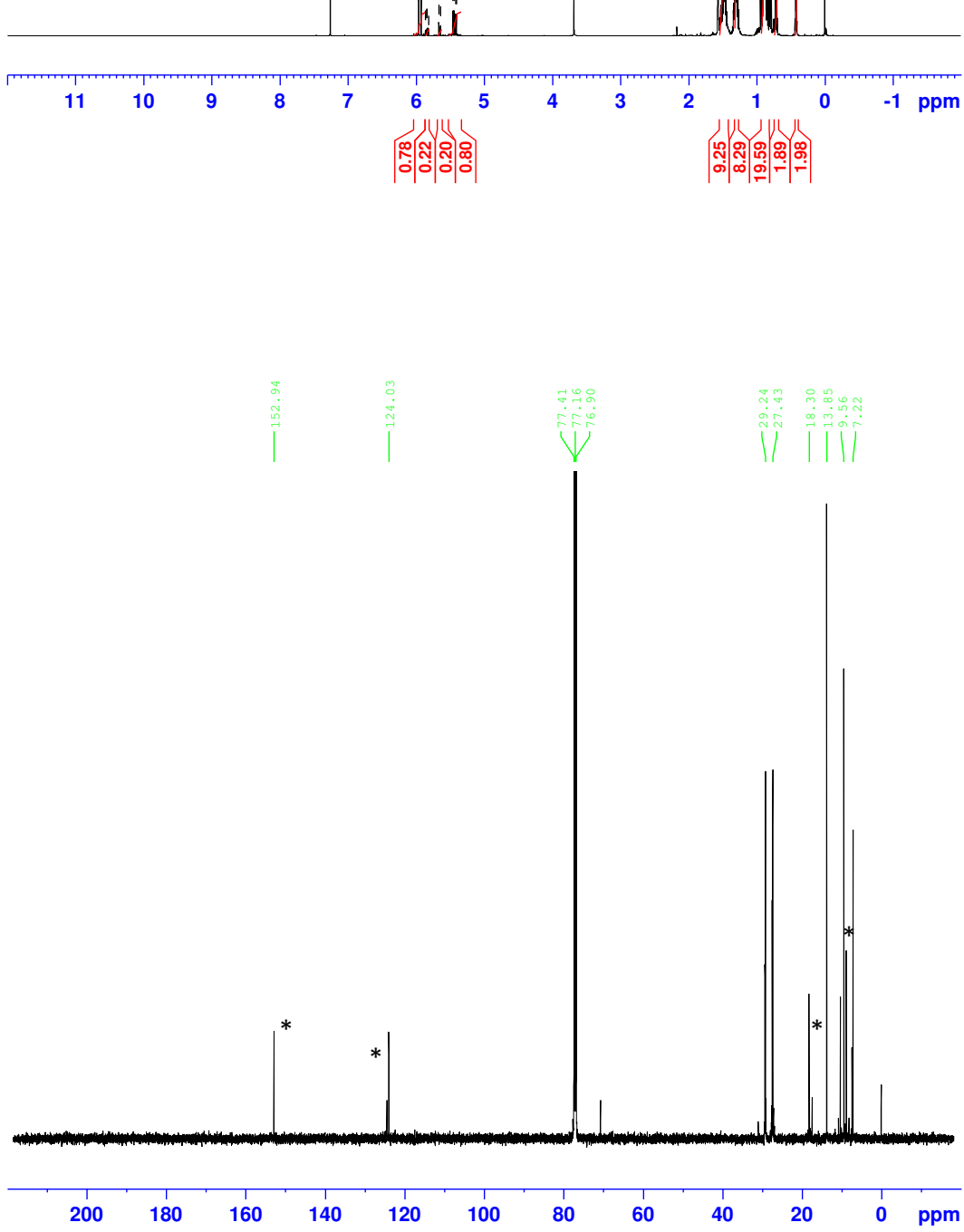

S-26 

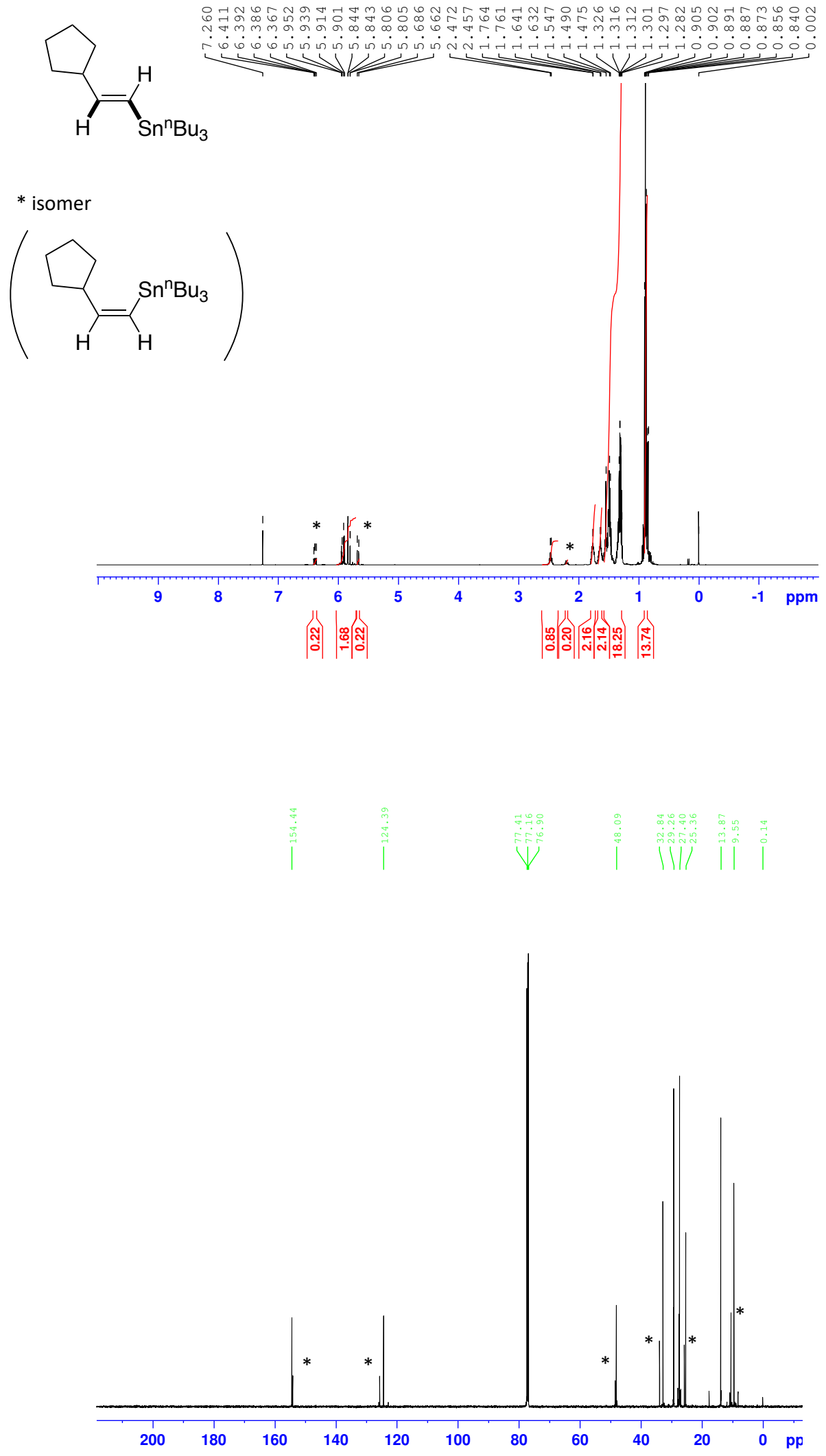

S-27 

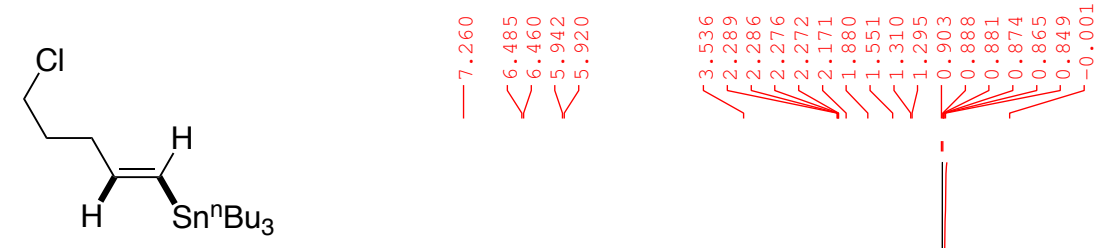

$\left({ }^{*}\right.$ isomer

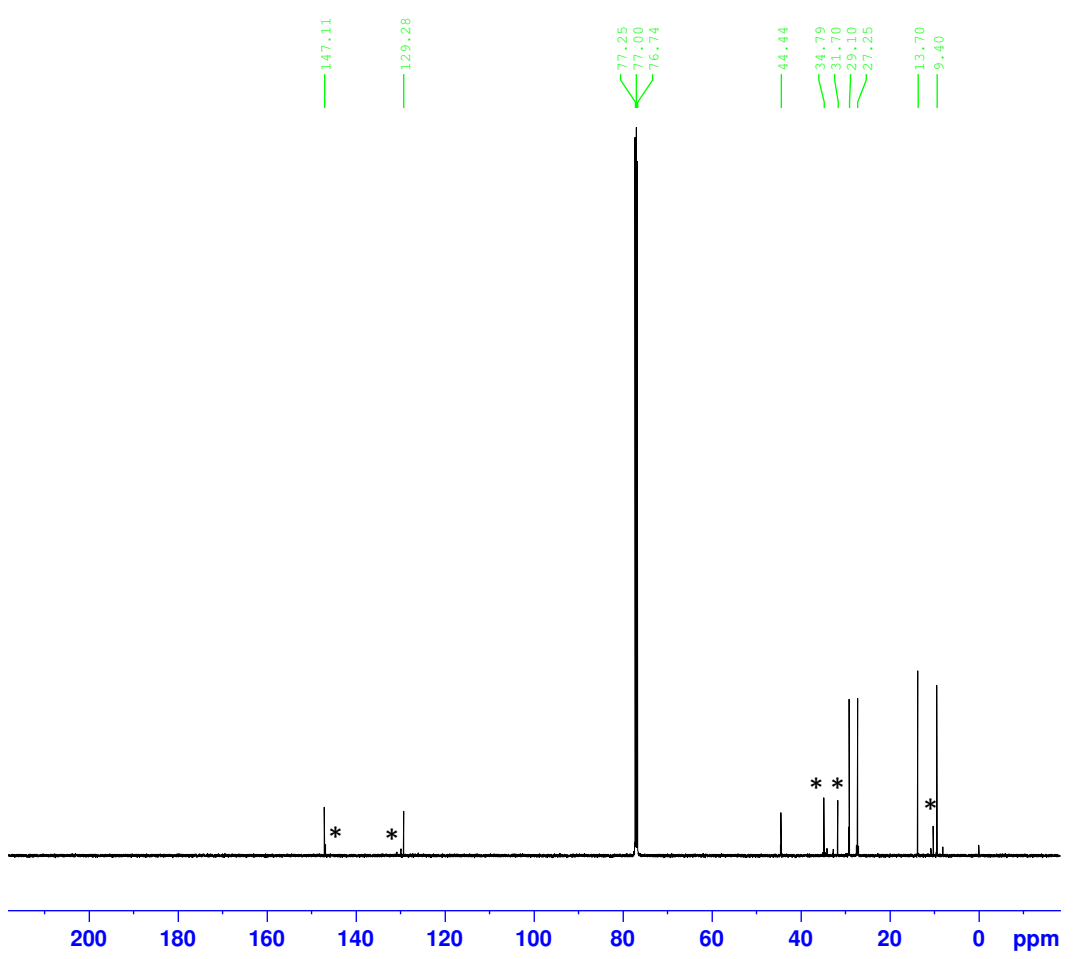



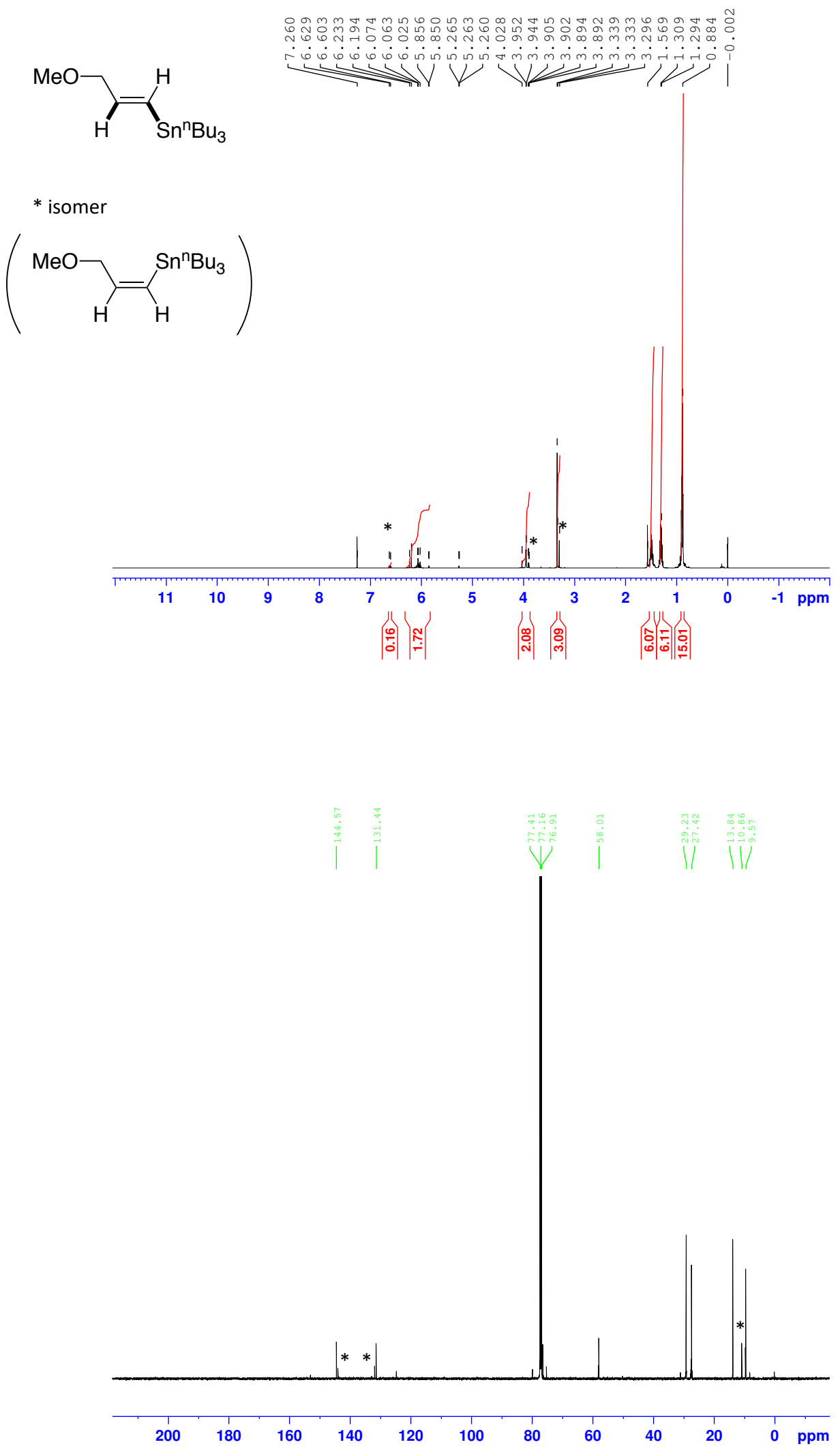

S-29 

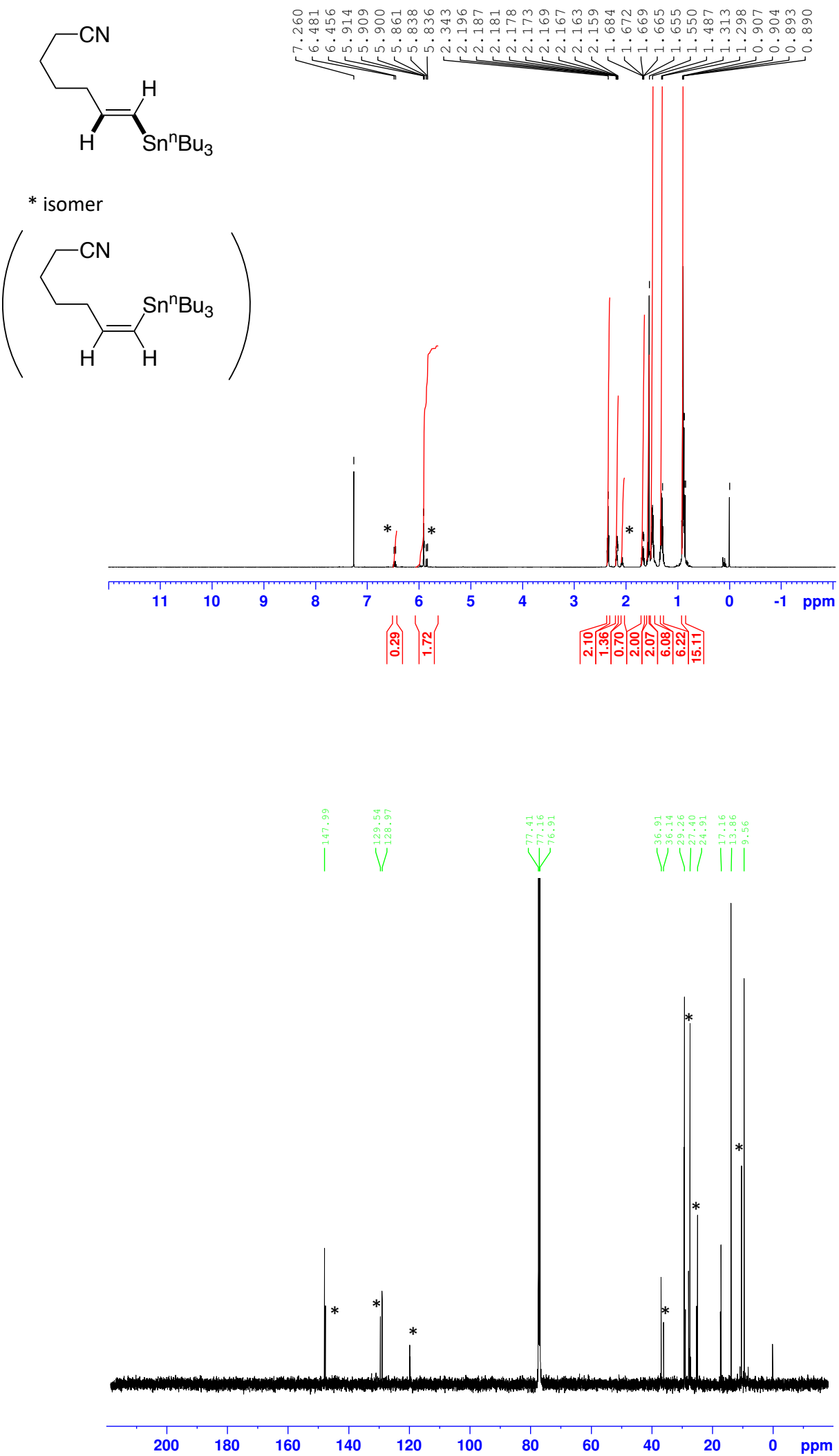

S-30 

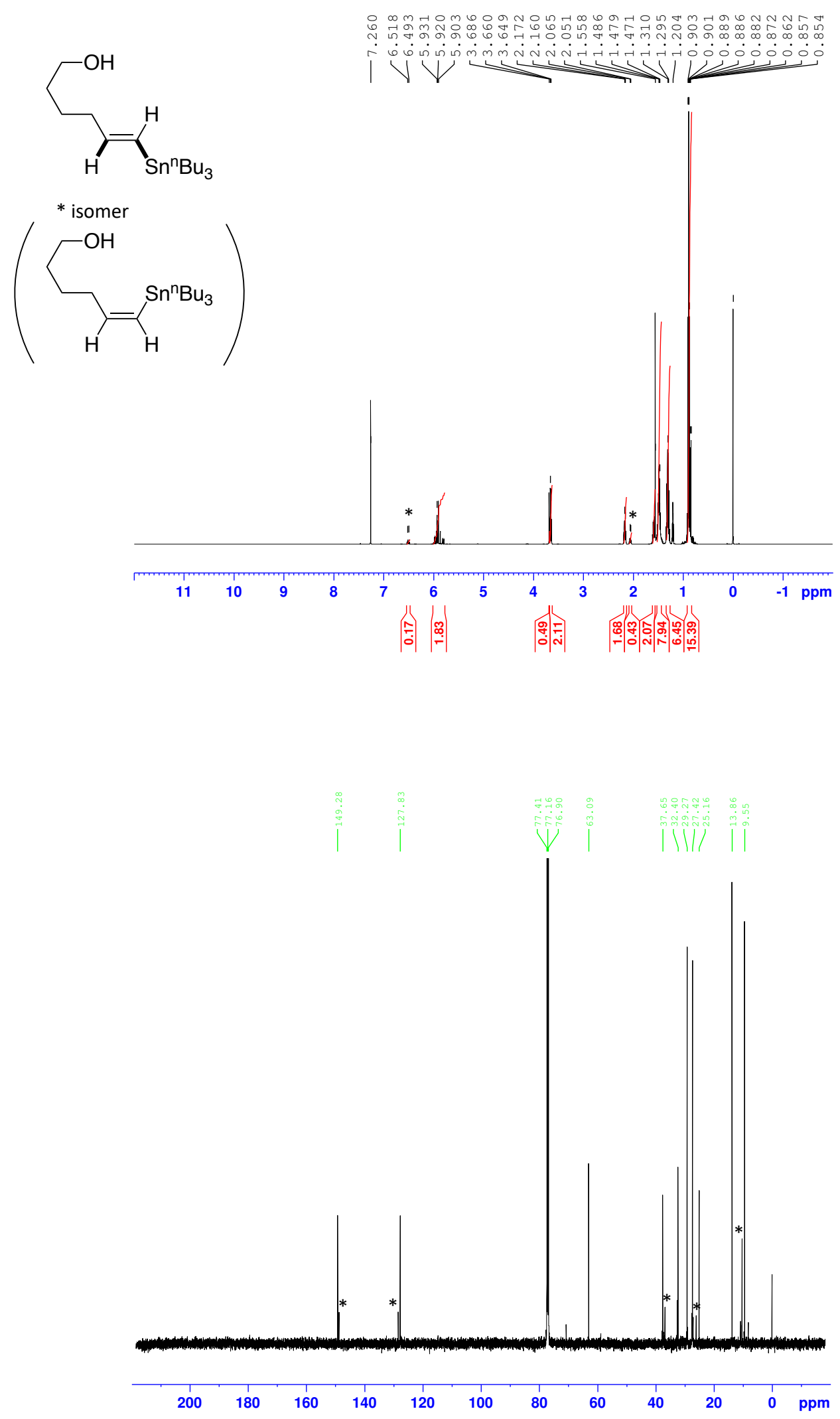

S-31 

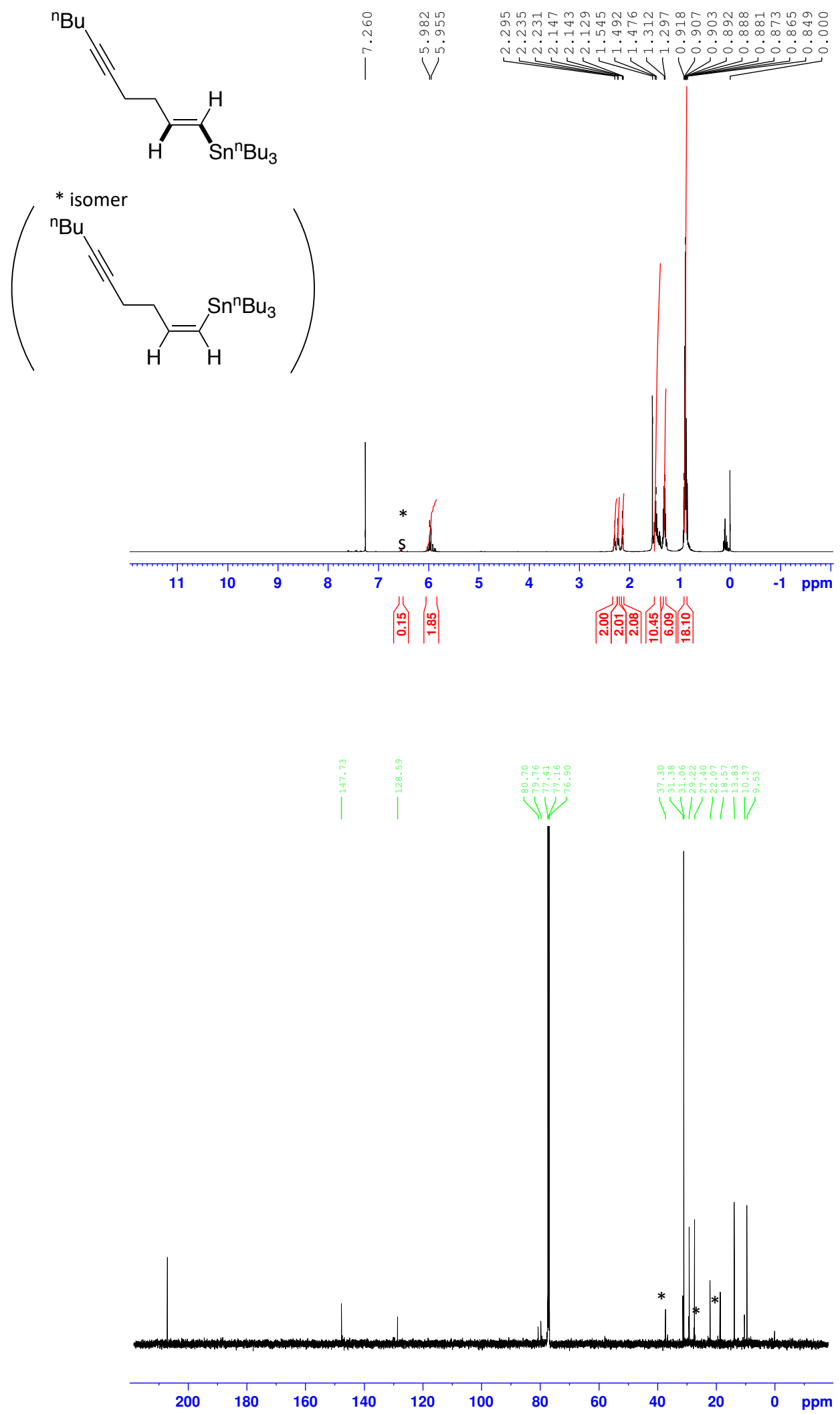

S-32 

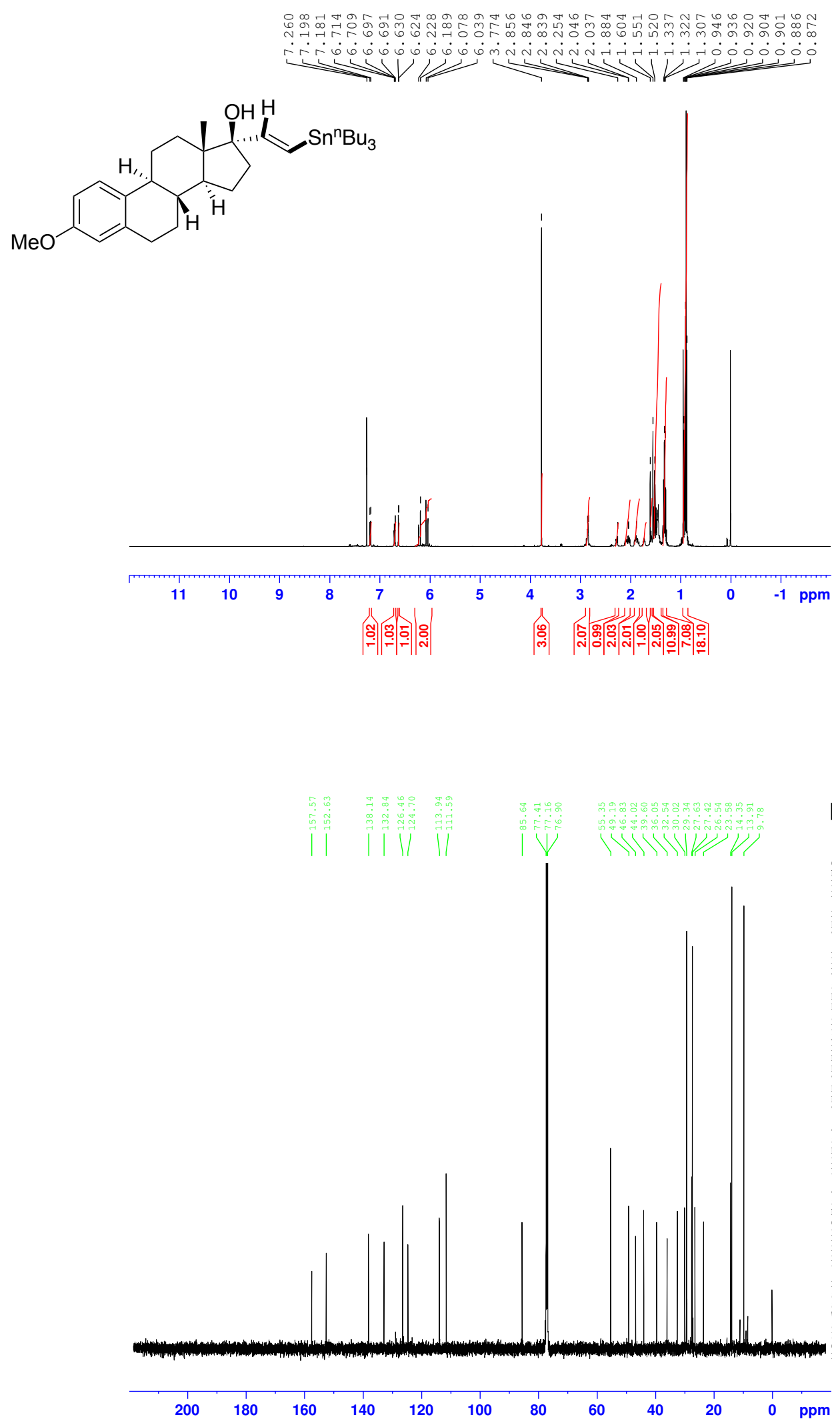

S-33 

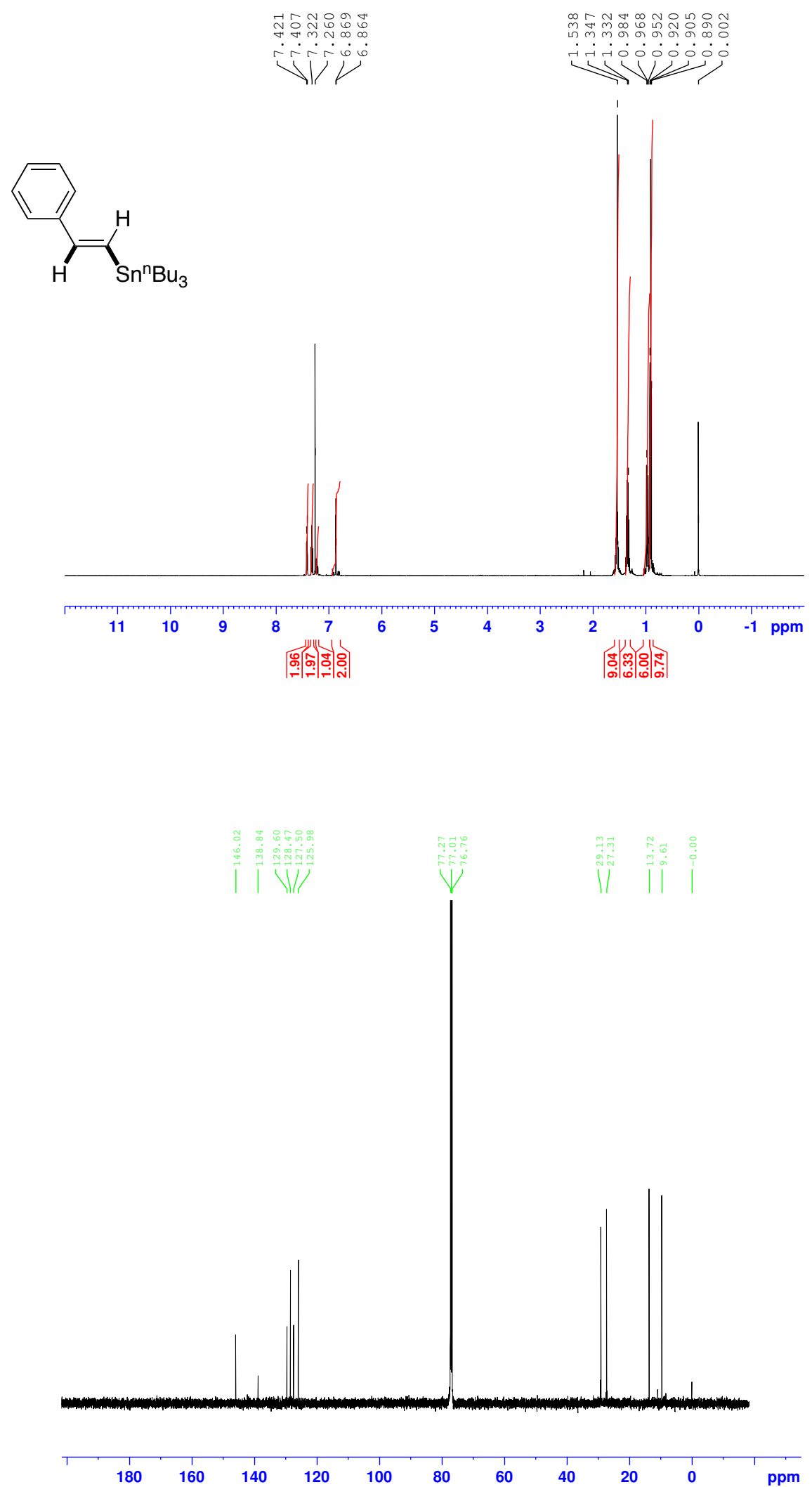

S-34 

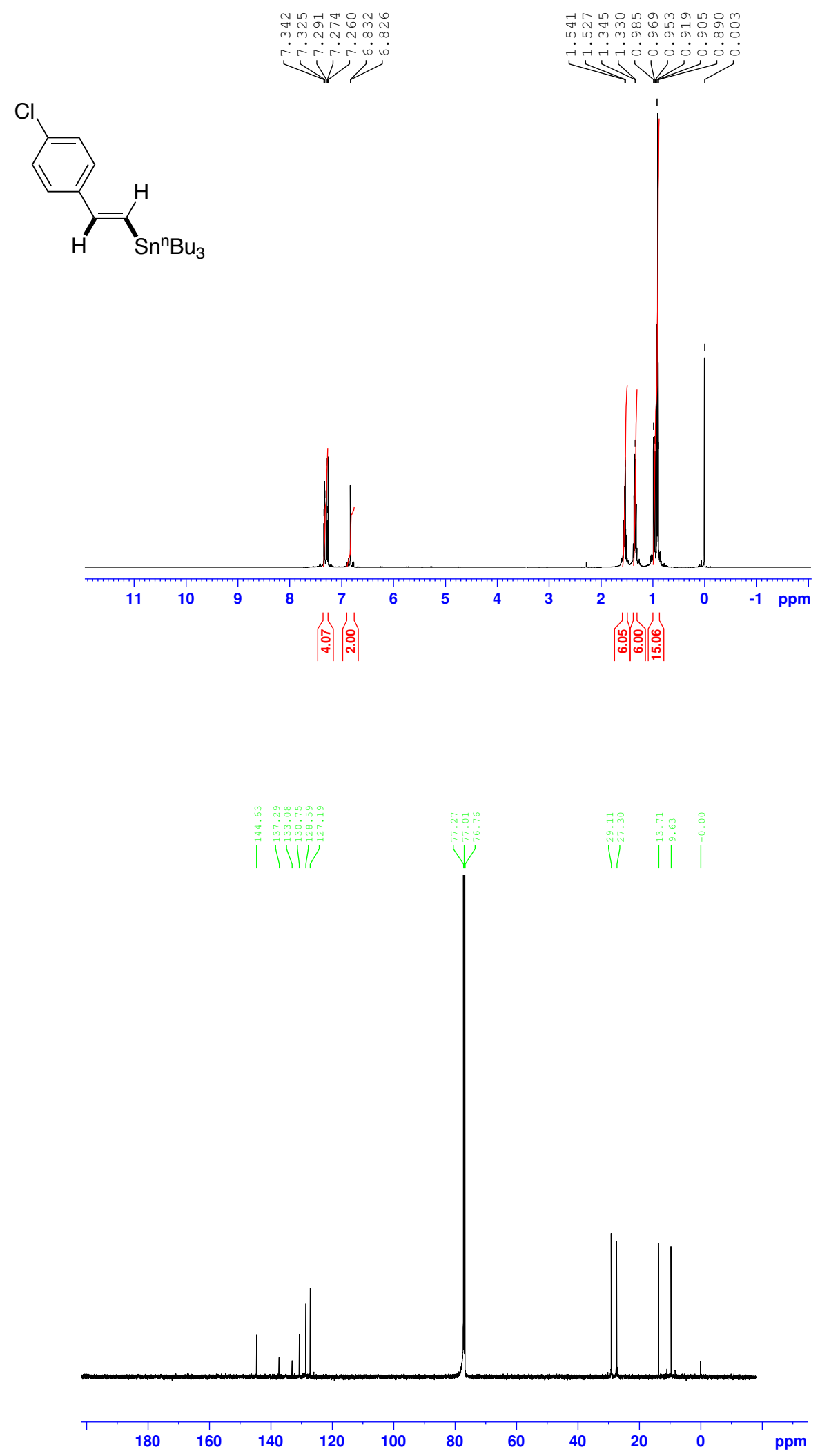

S-35 

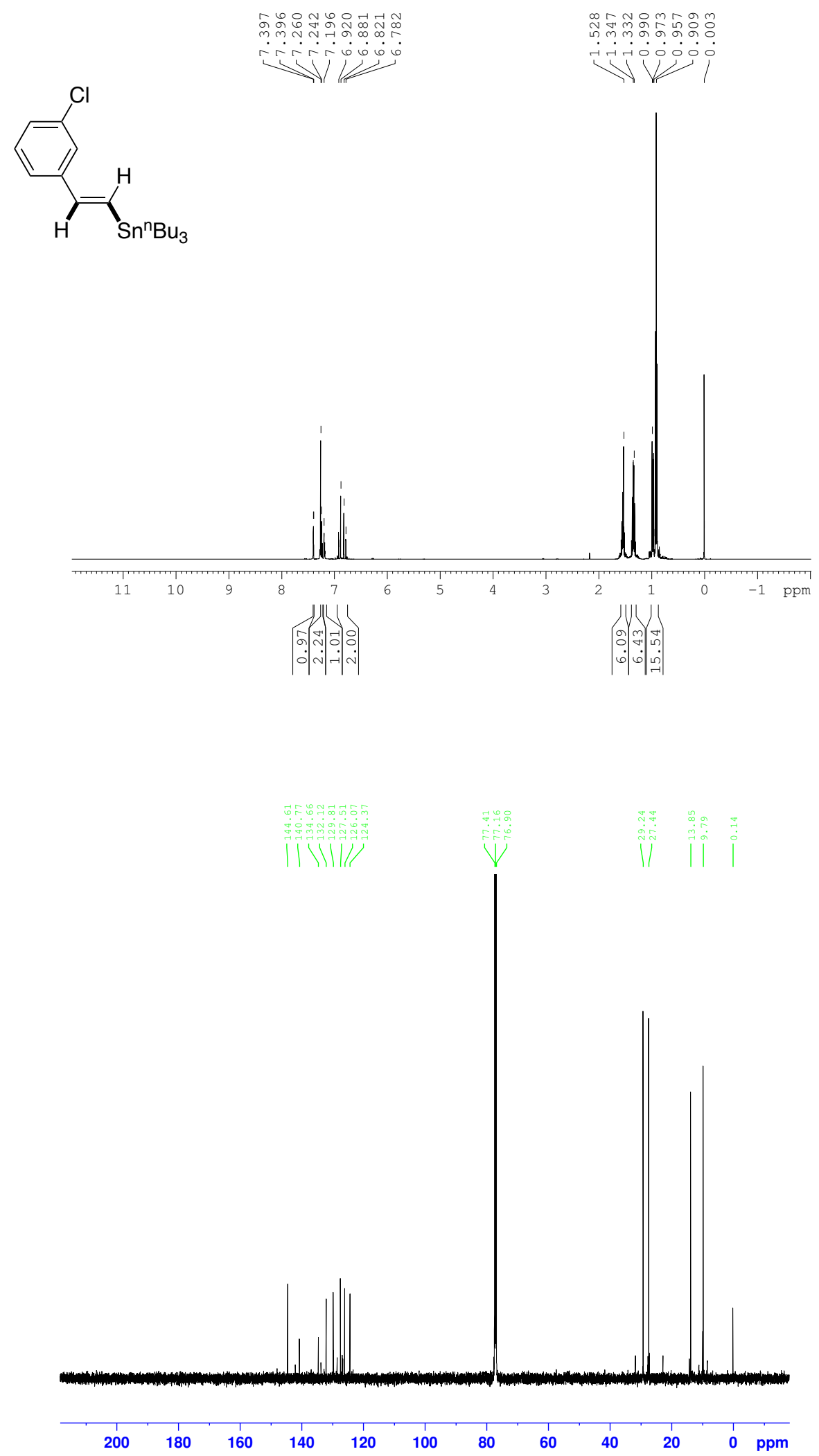

S-36 

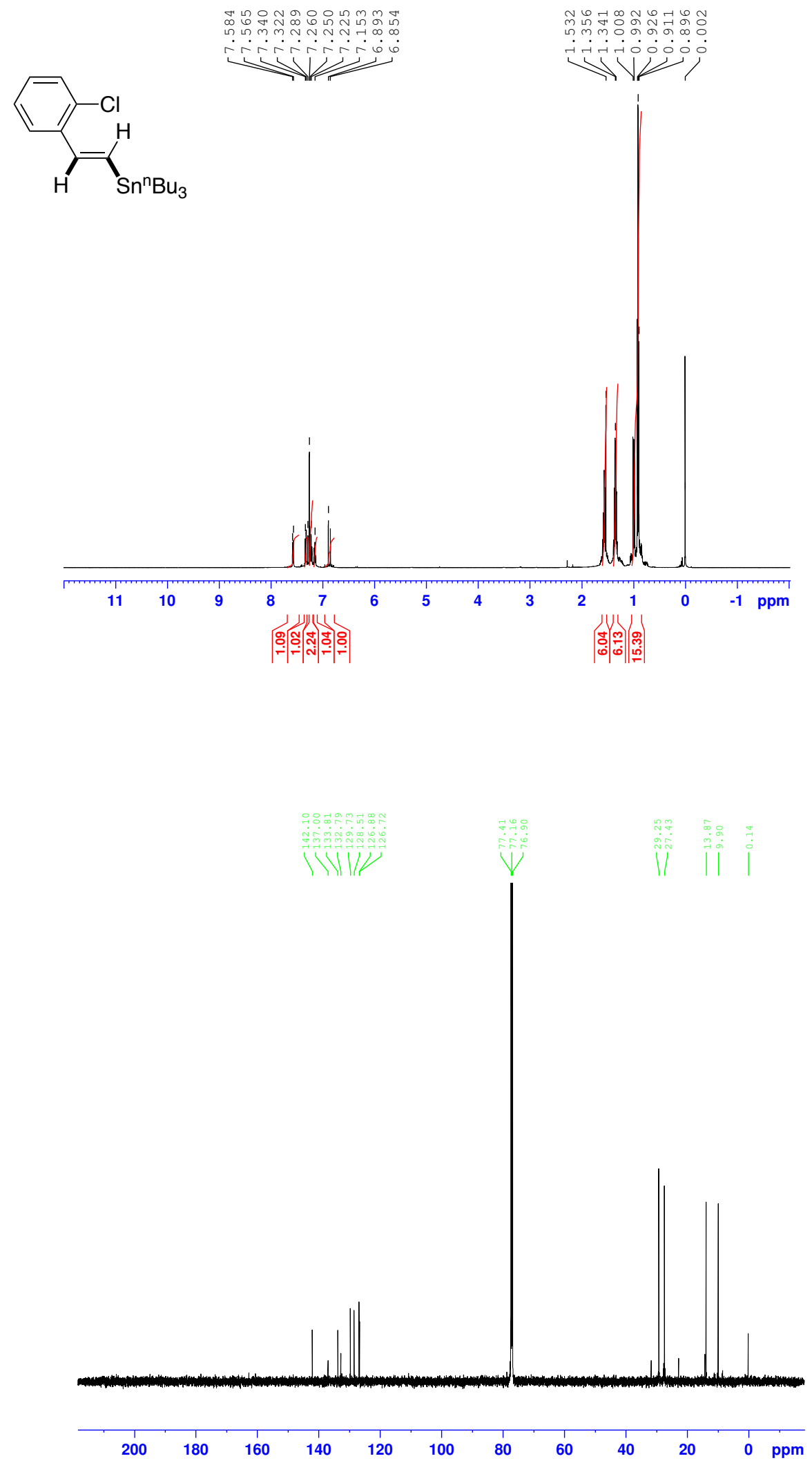

S-37 

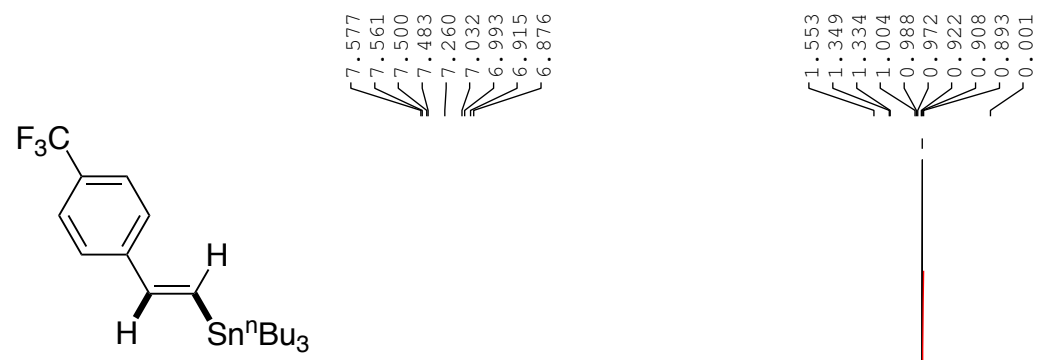

* ${ }^{n} \mathrm{Bu}_{3} \mathrm{Sn}-\mathrm{Sn}^{\mathrm{n}} \mathrm{Bu}_{3}$
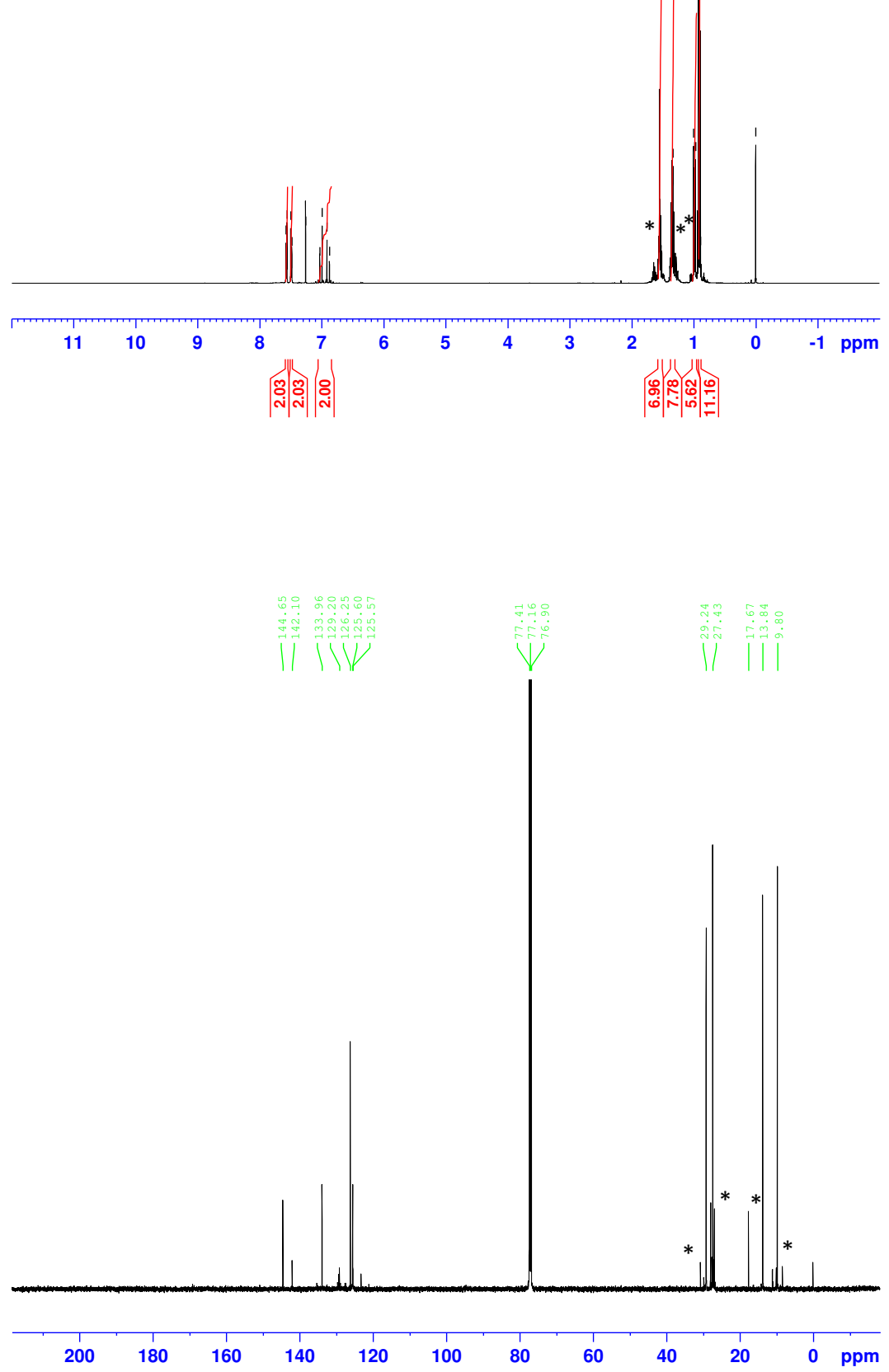

S-38 

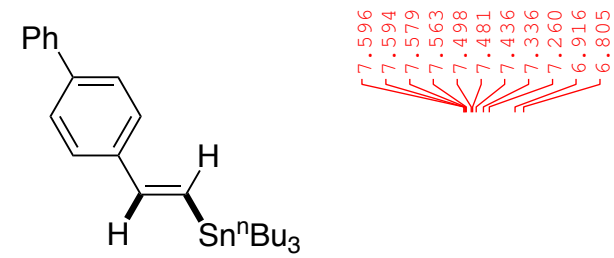

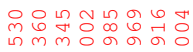

संखiji

$\left({ }_{H}^{*}\right.$
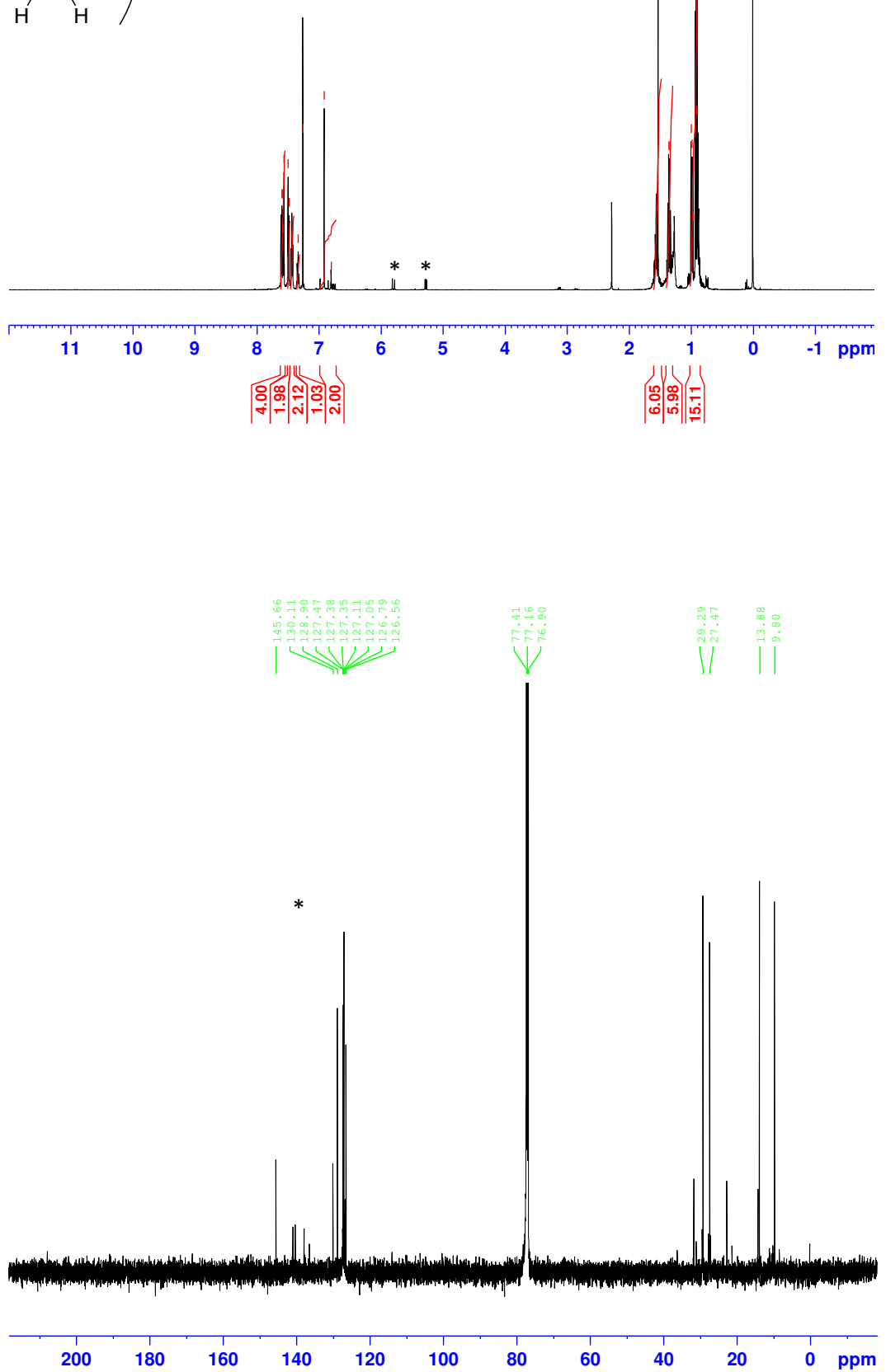

S-39 

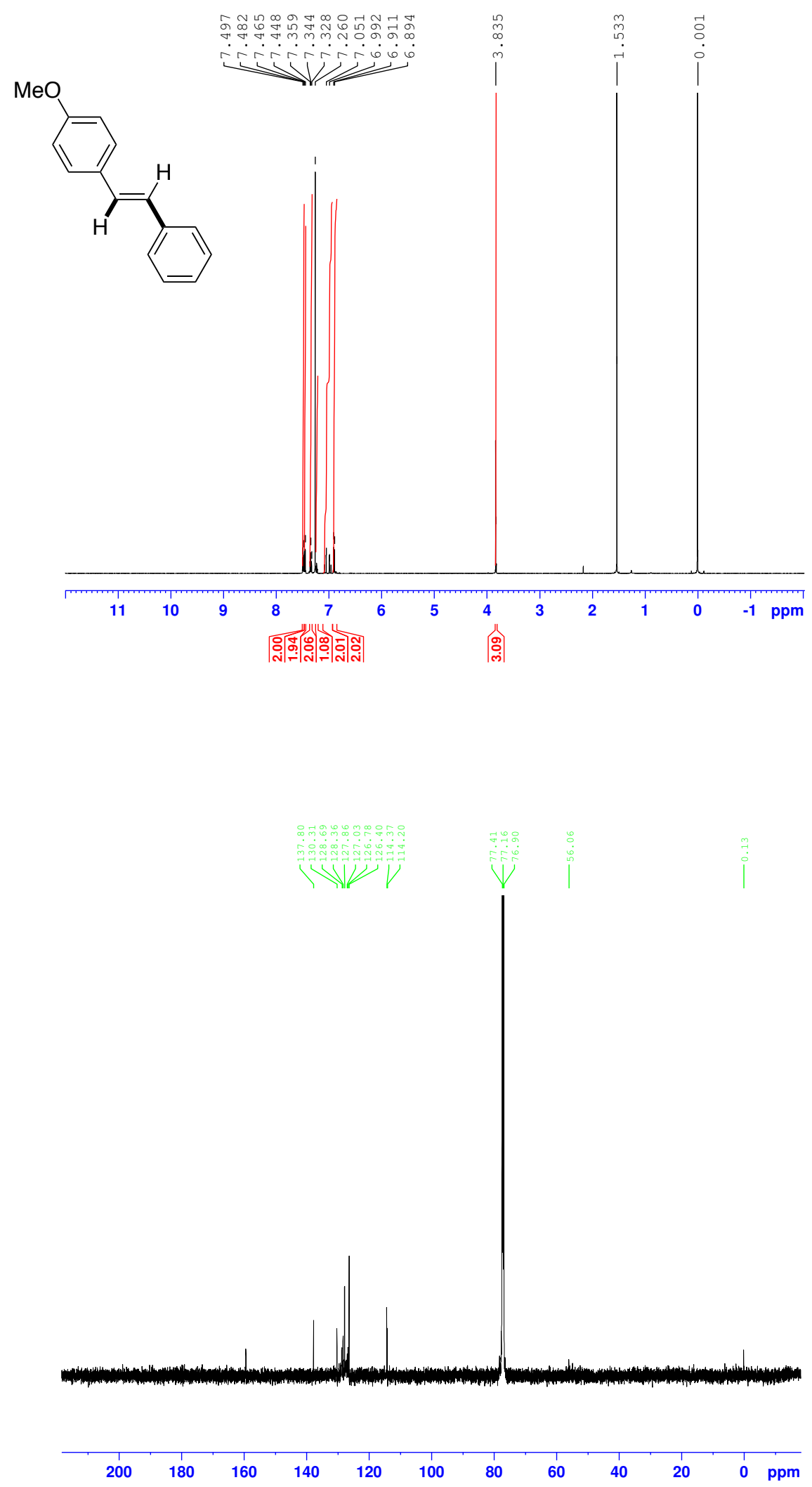

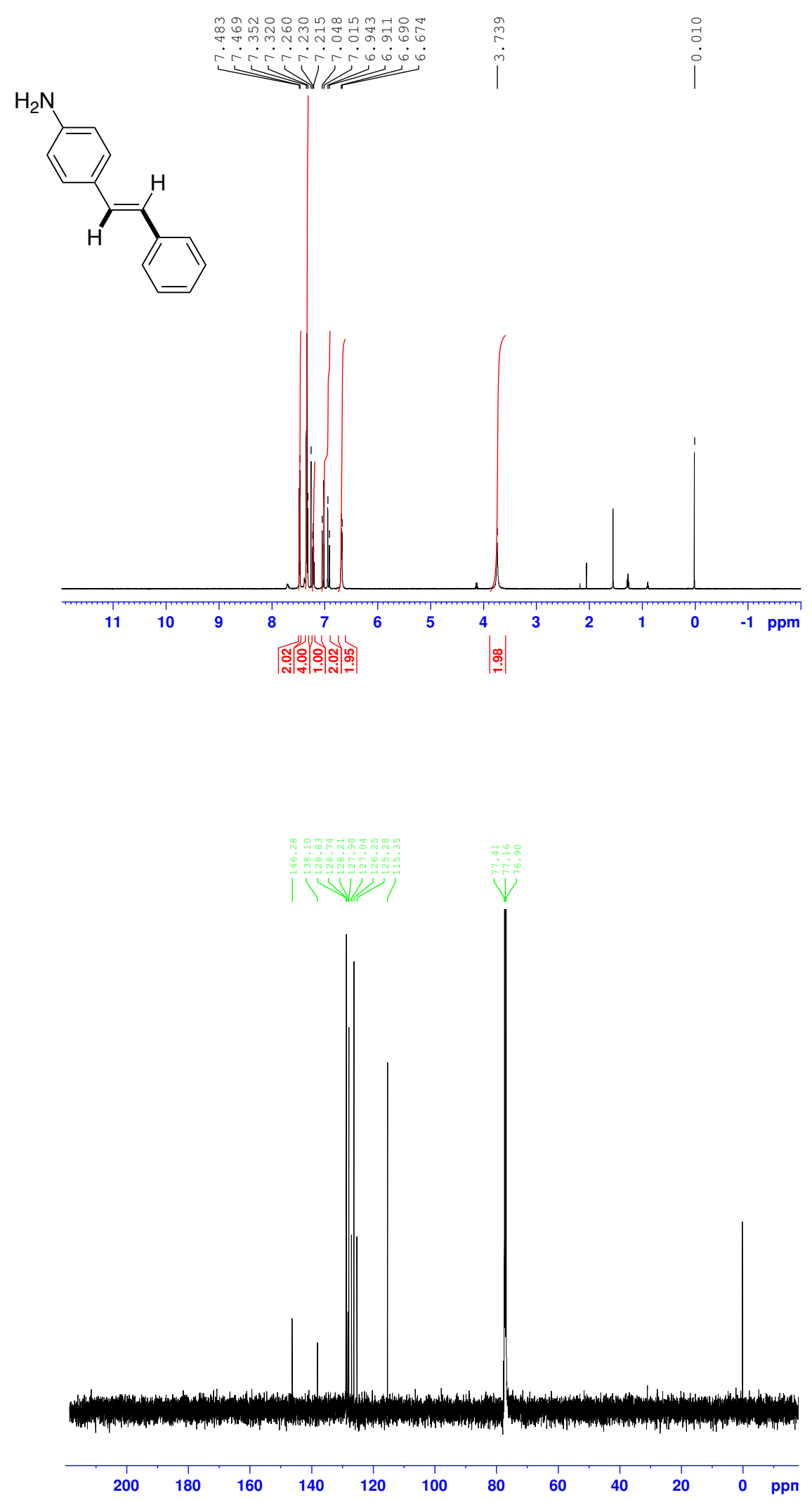

\section{S-41}



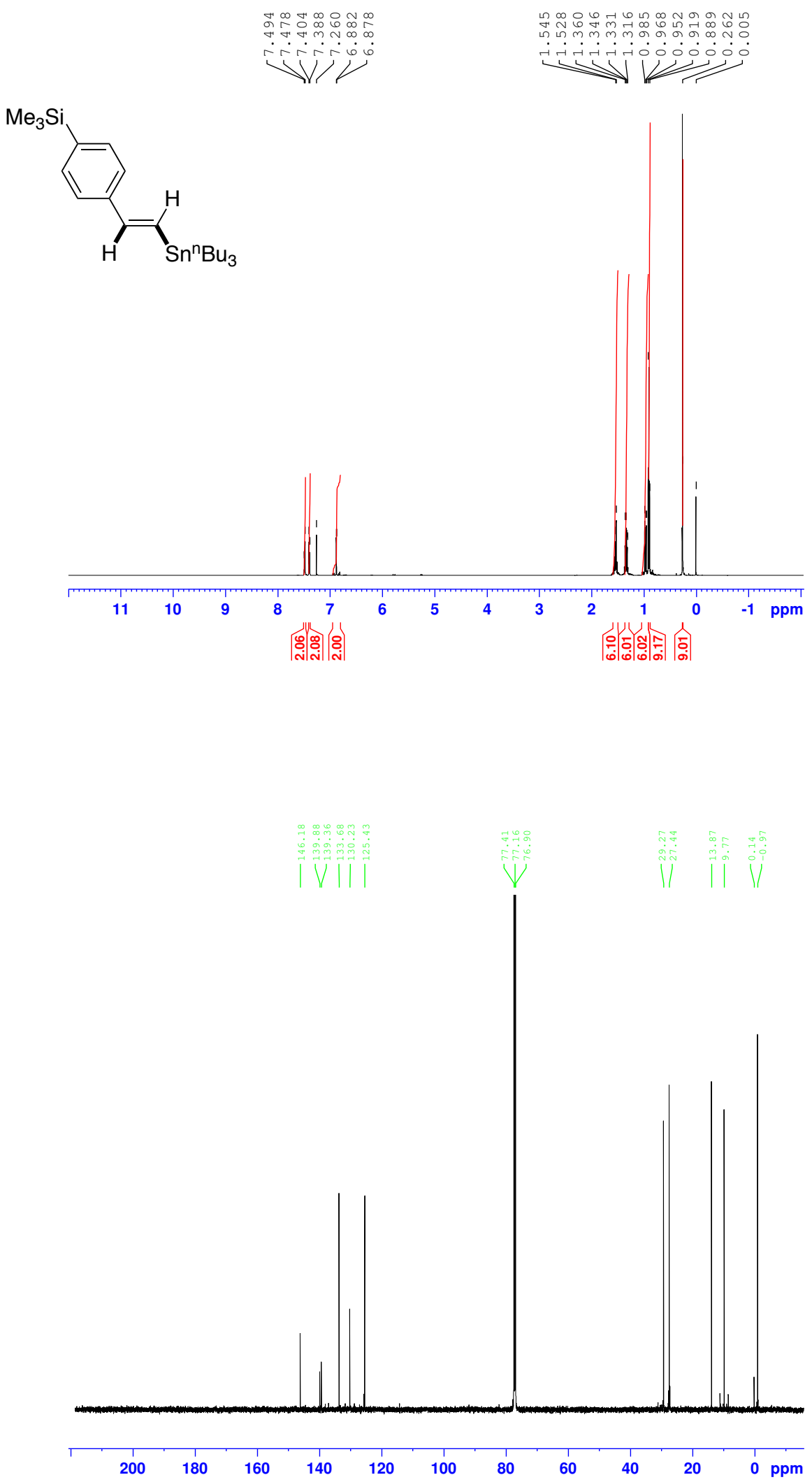

S-42 

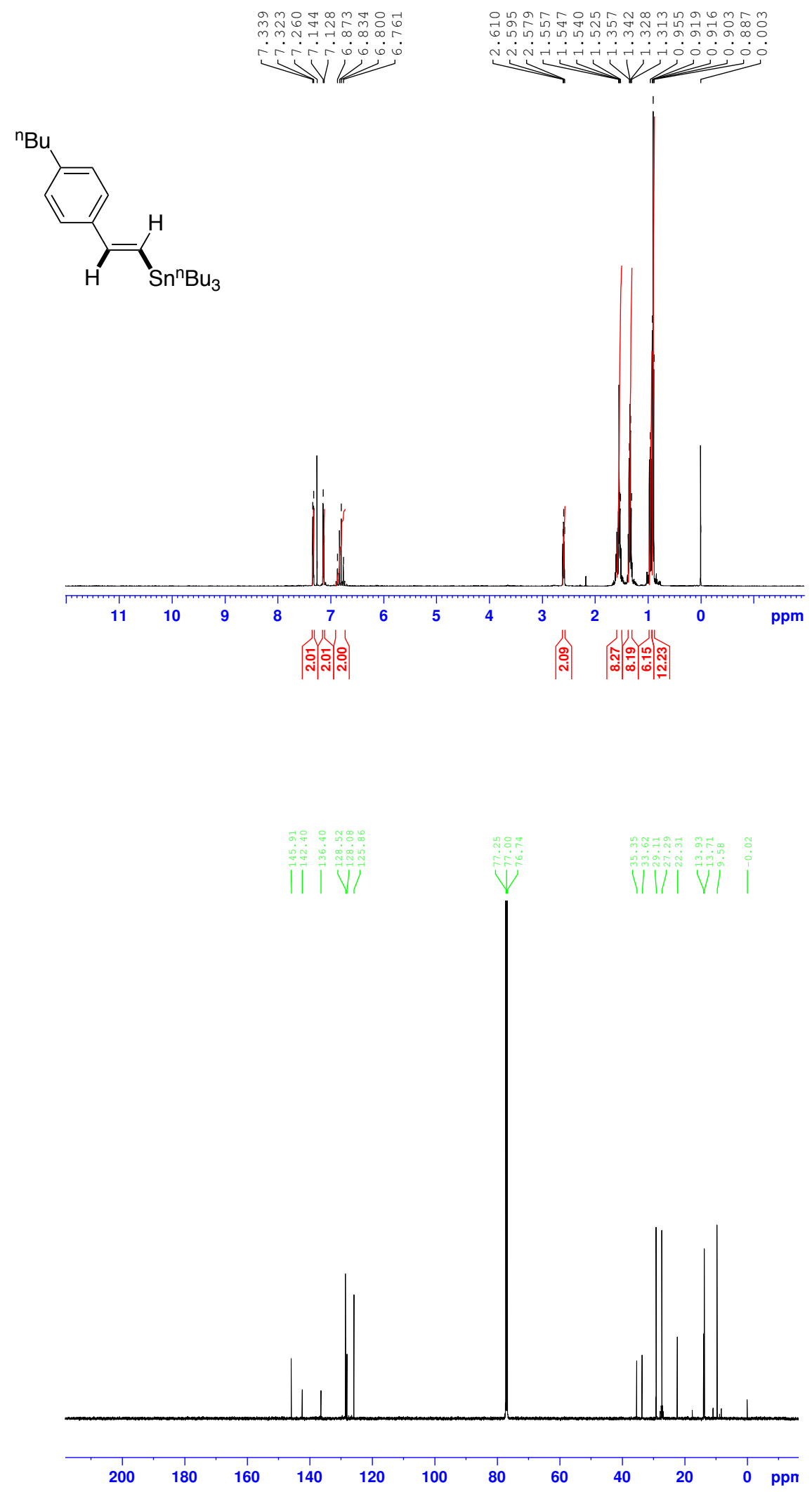

S-43 

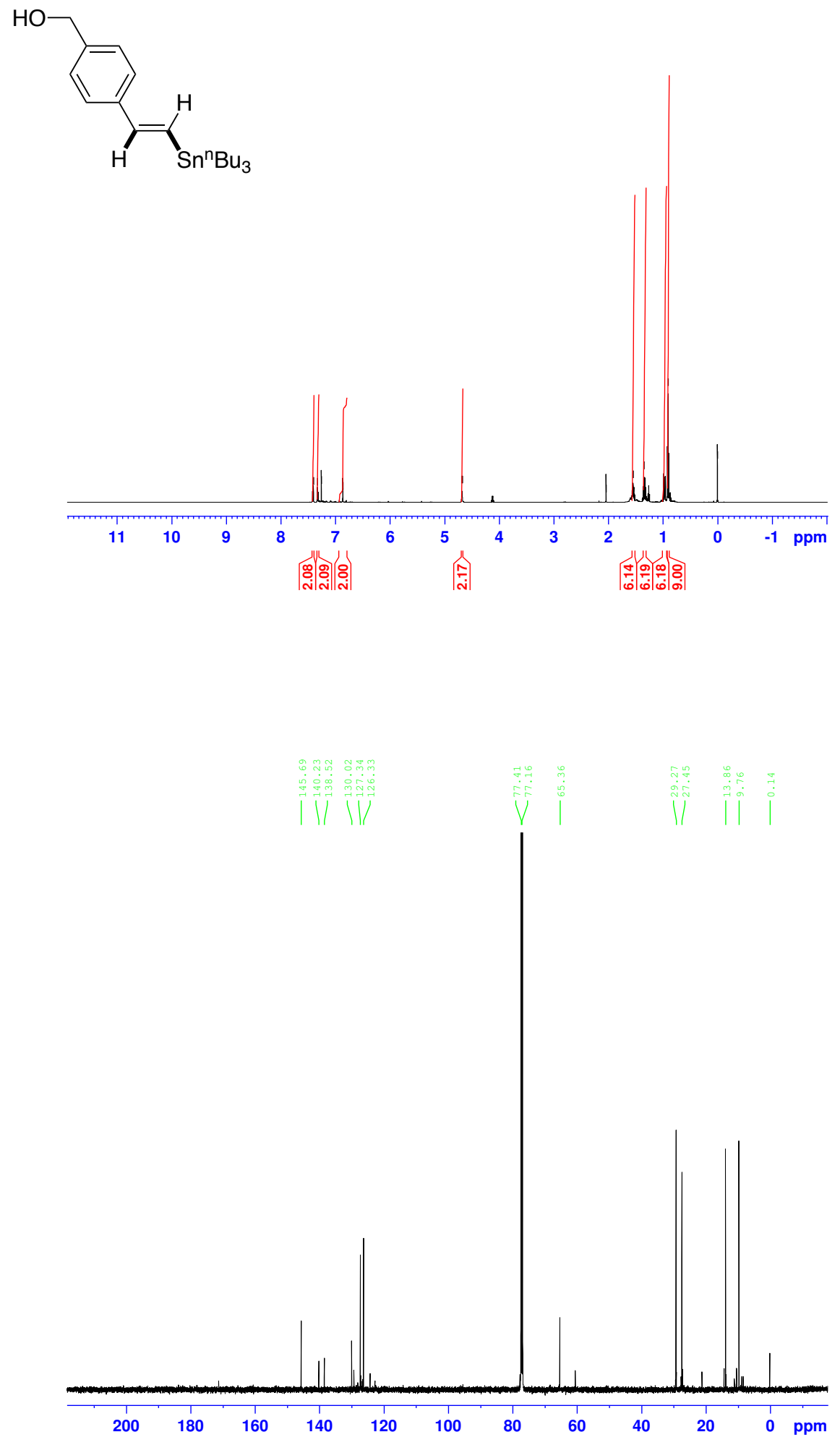

\section{S-44}



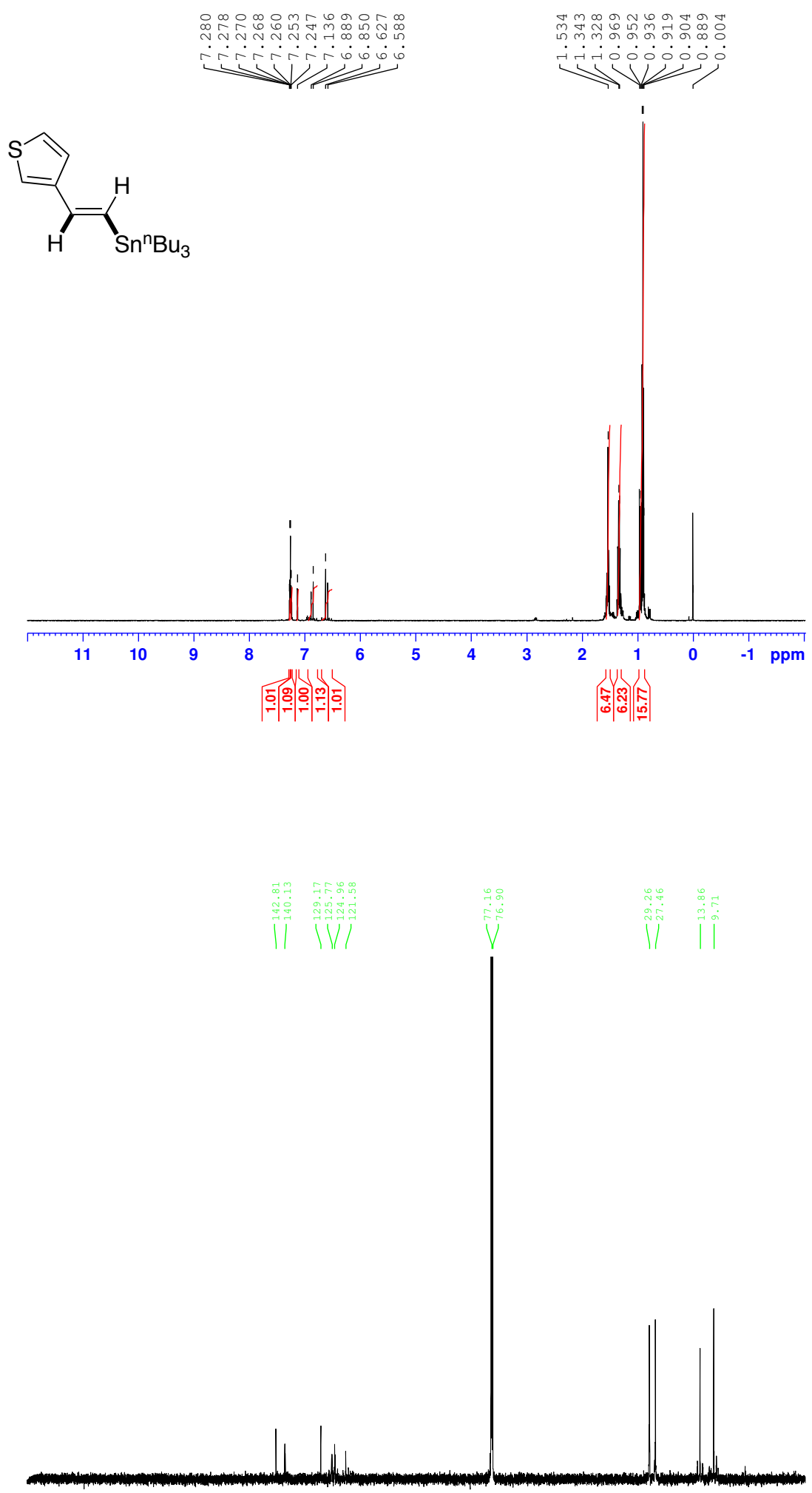

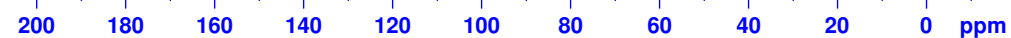



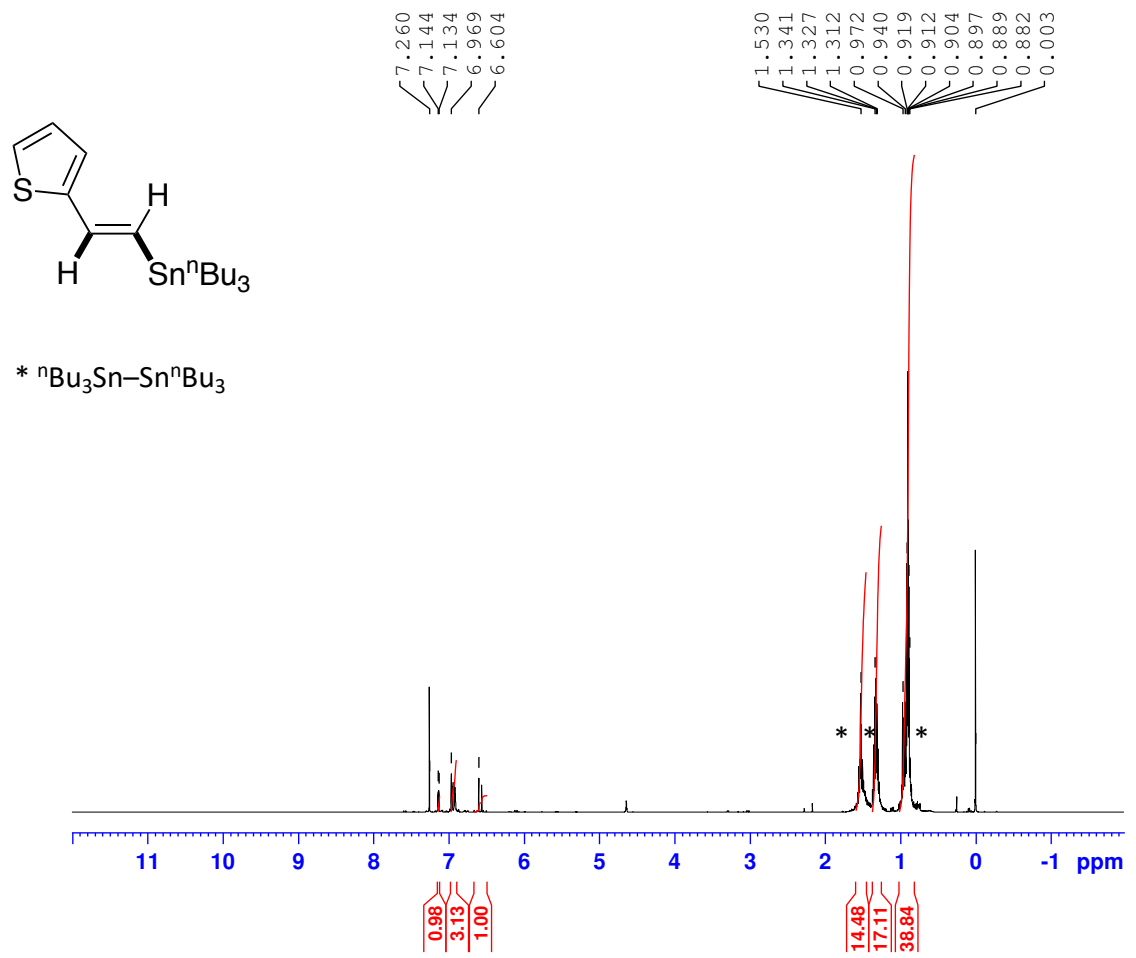

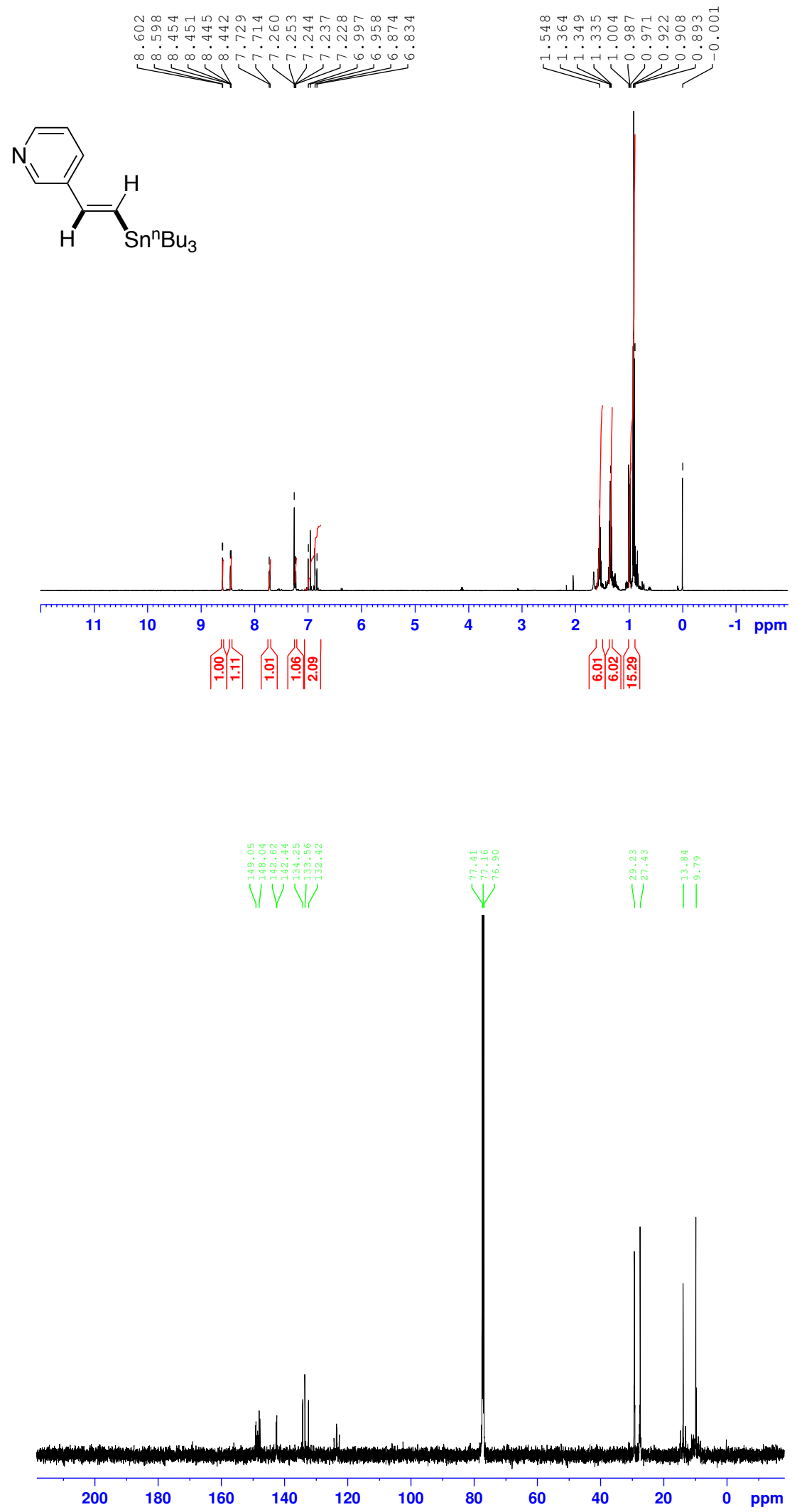

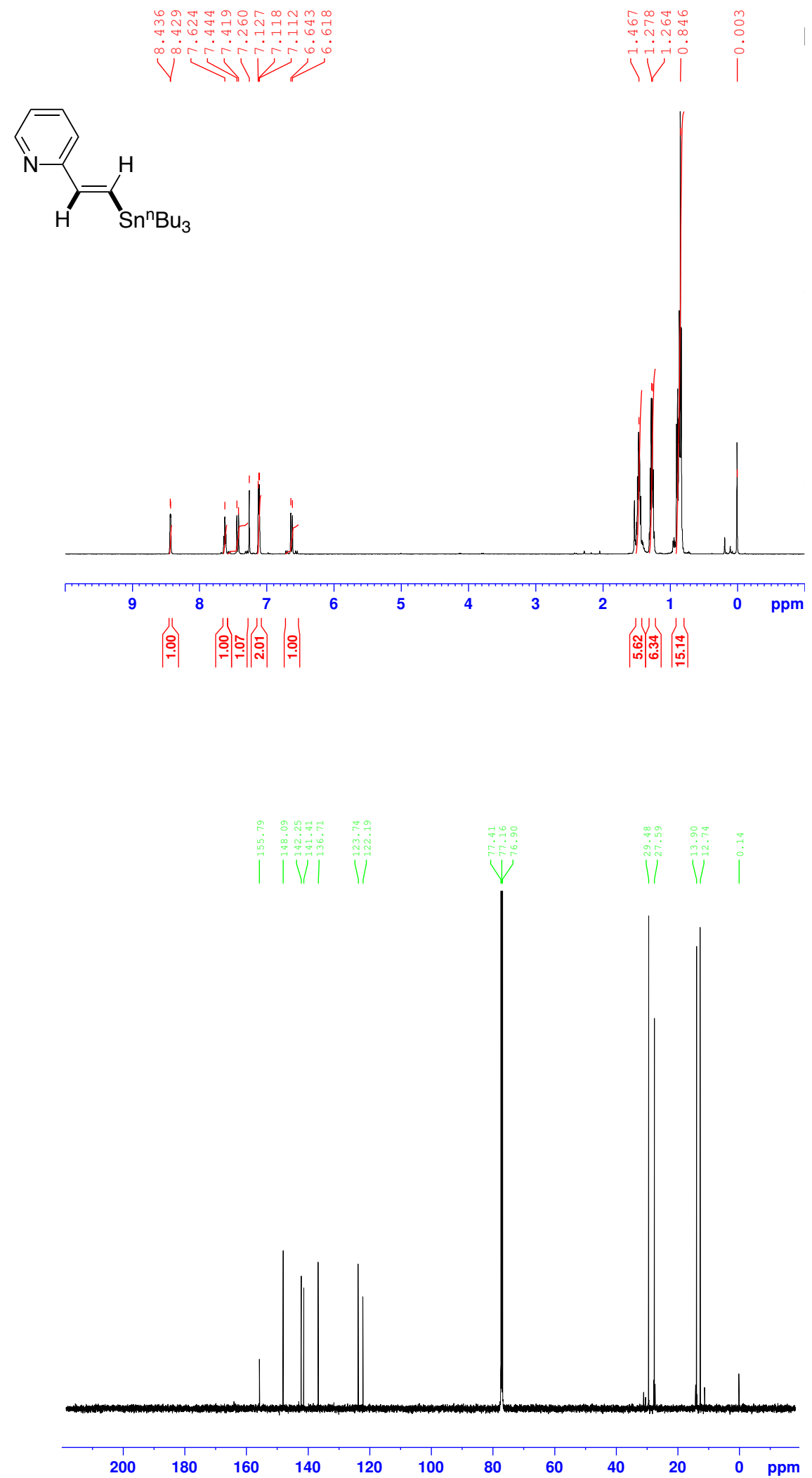

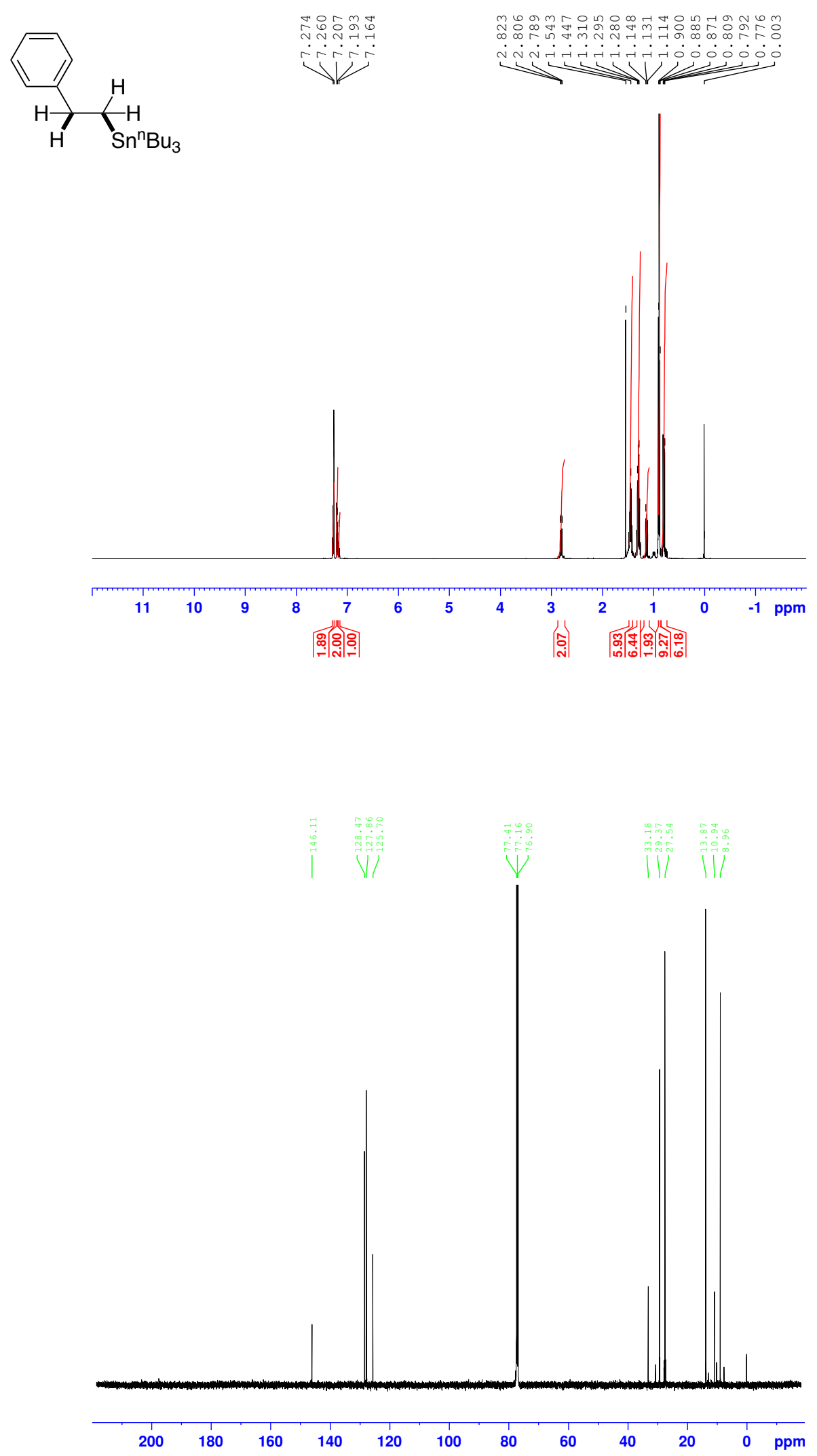

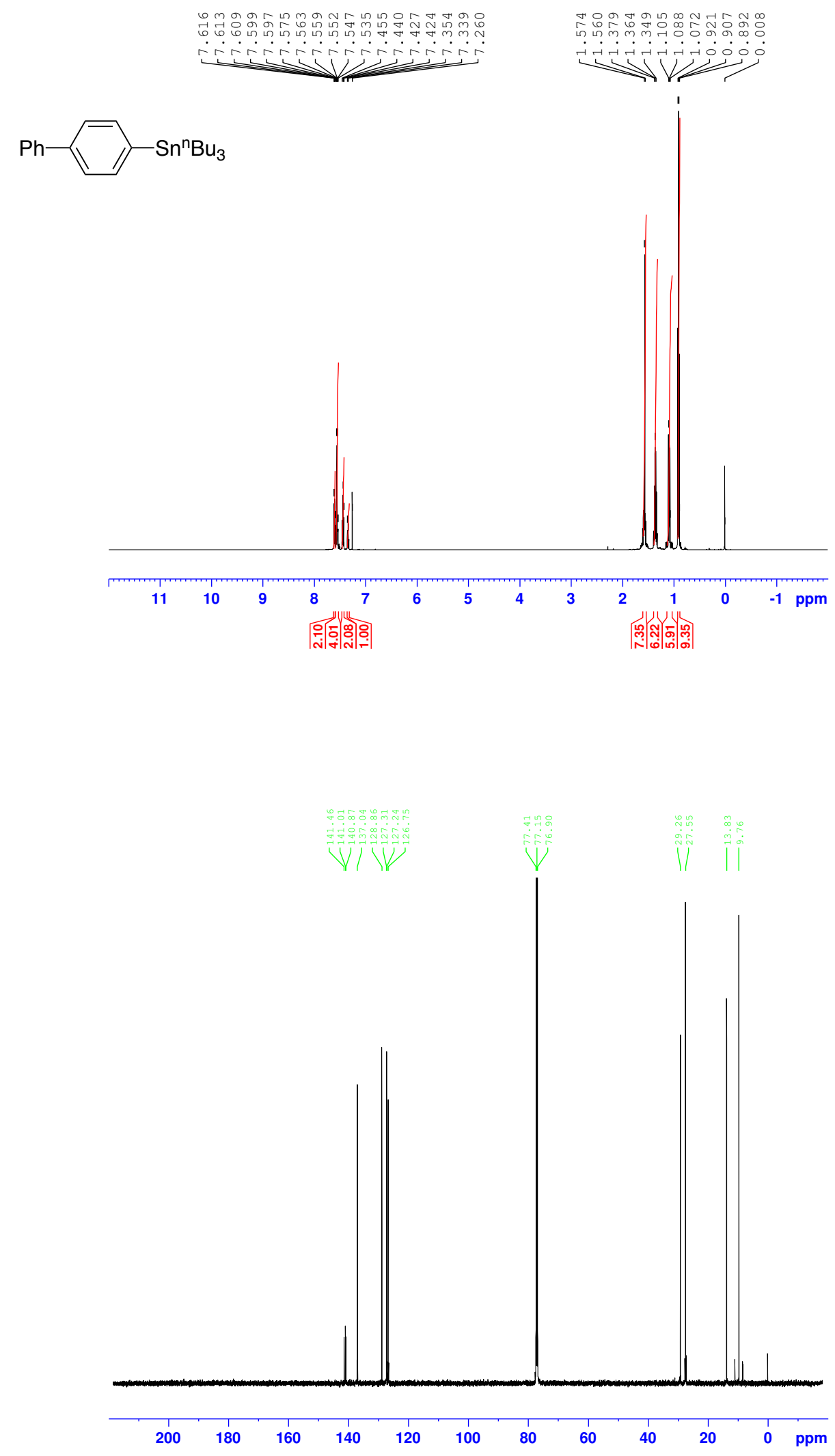

S-50 

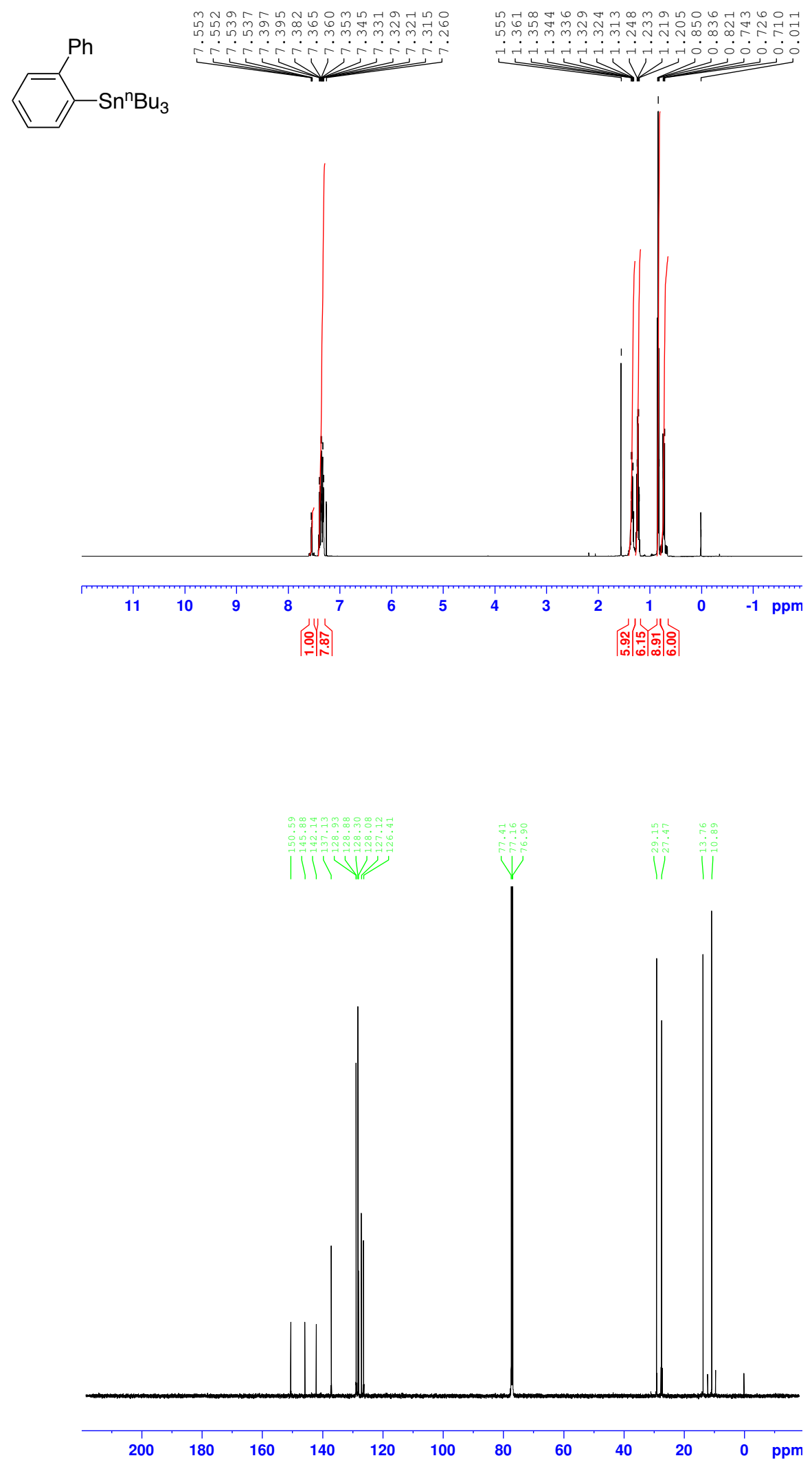

S-51 

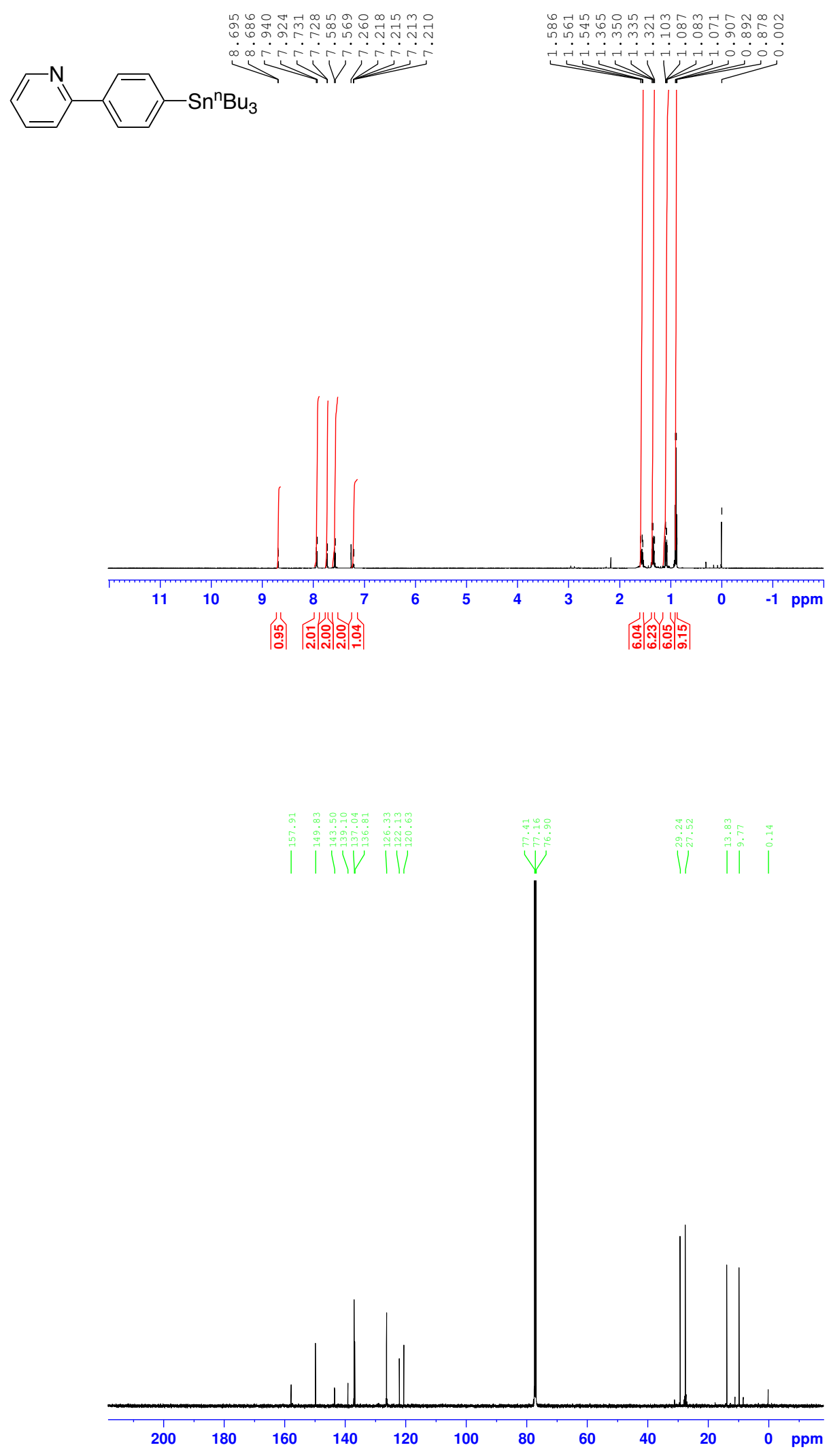

S-52 

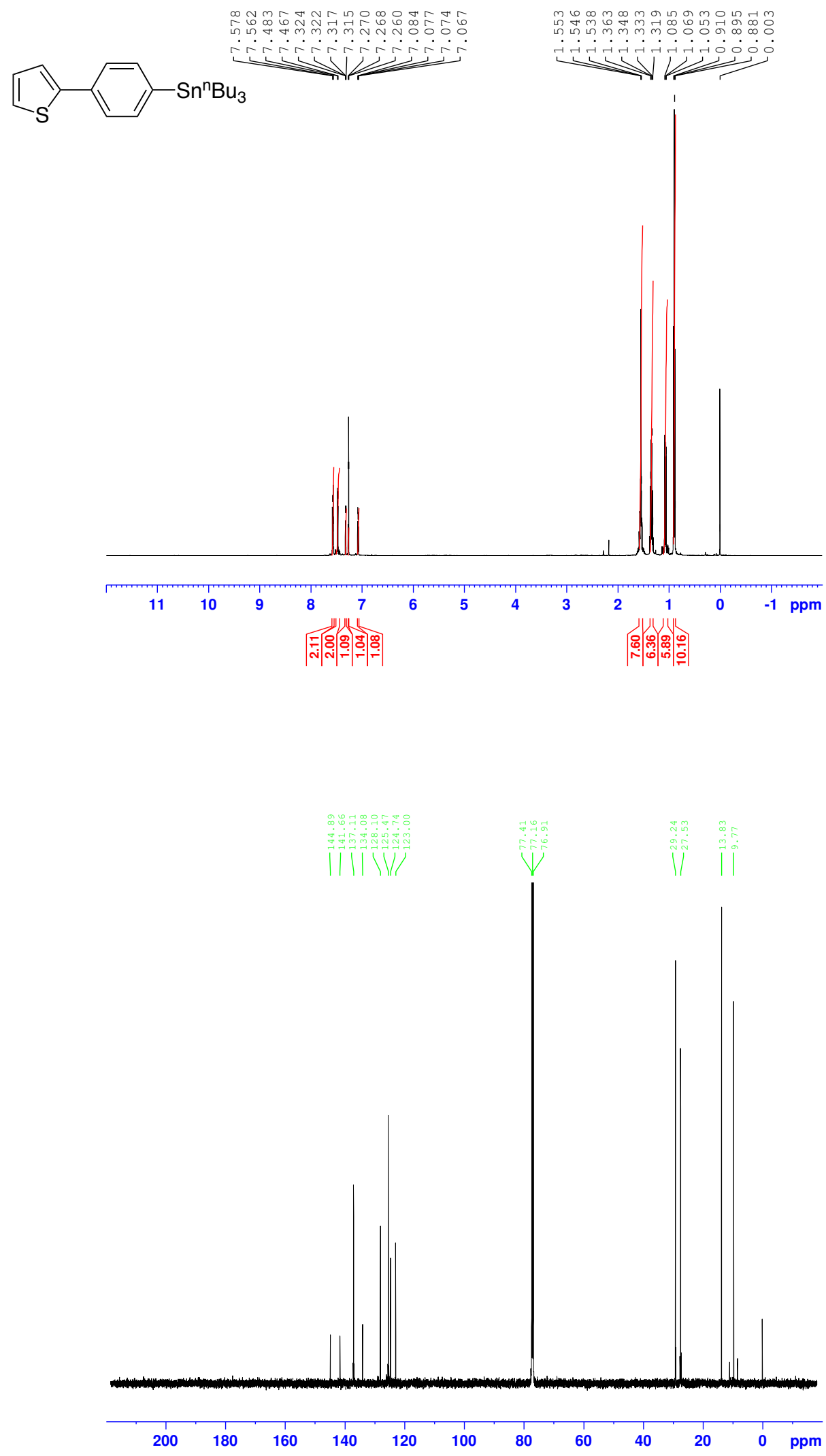

S-53 

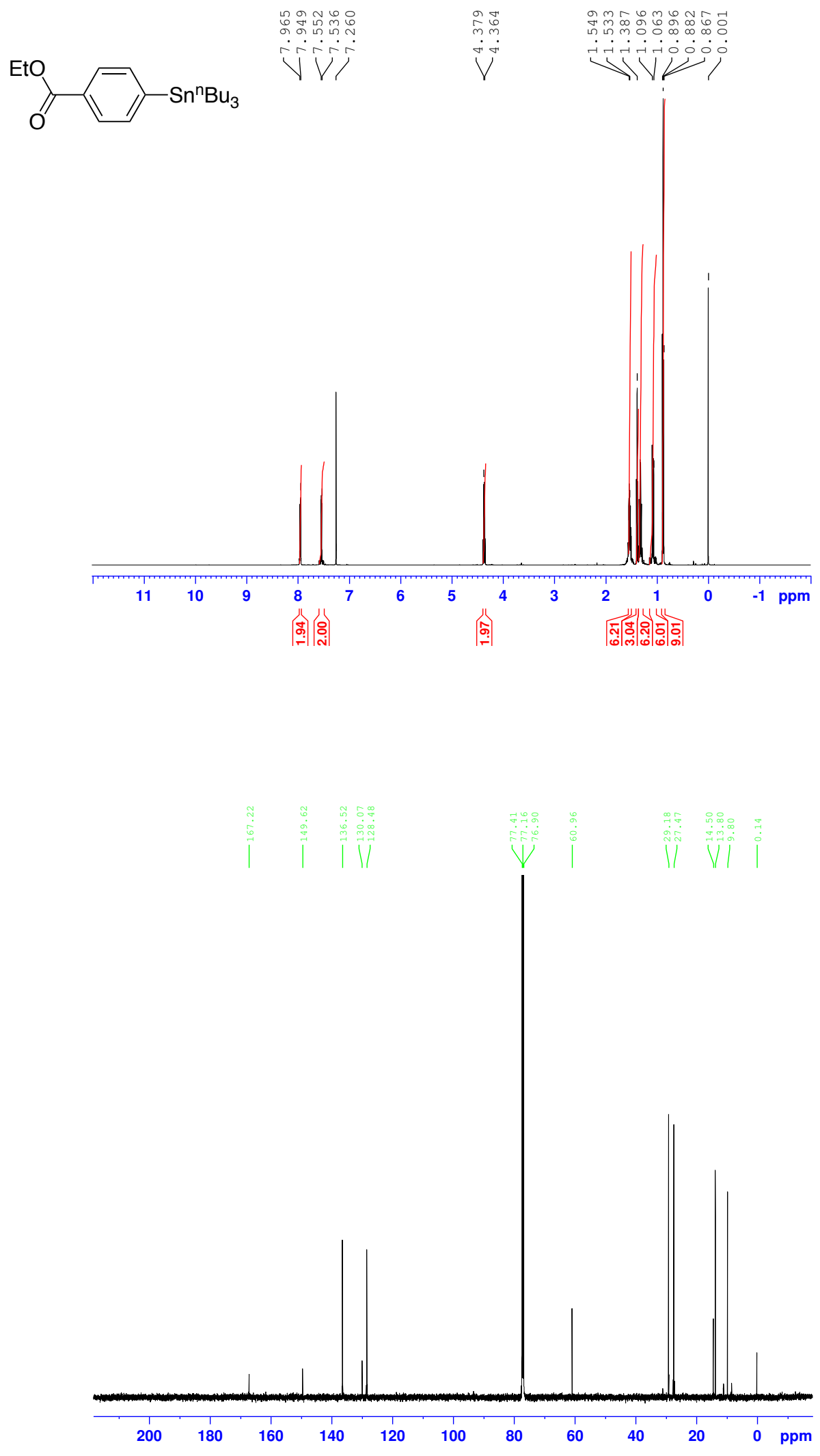

\section{S-54}



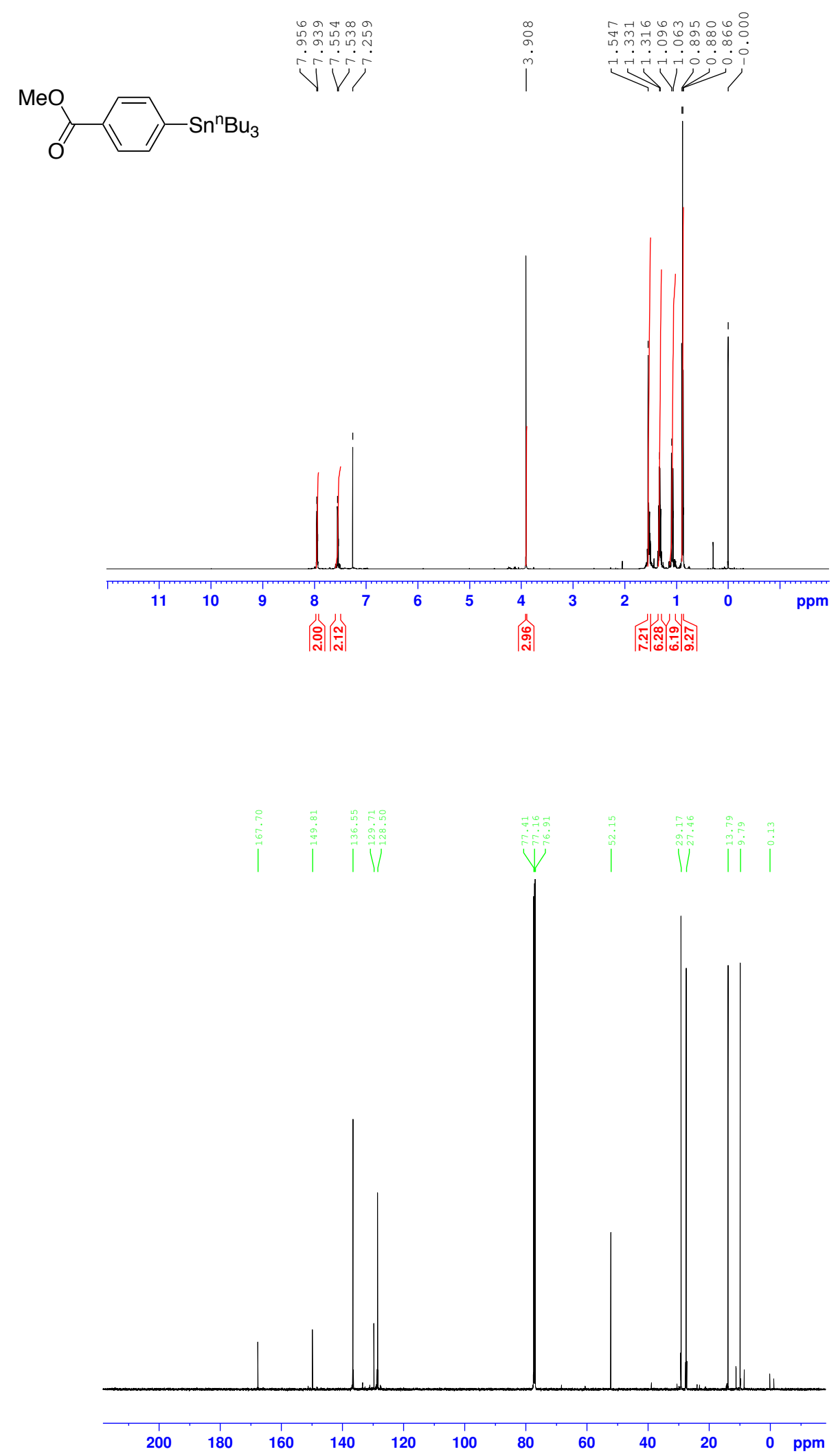

S-55 

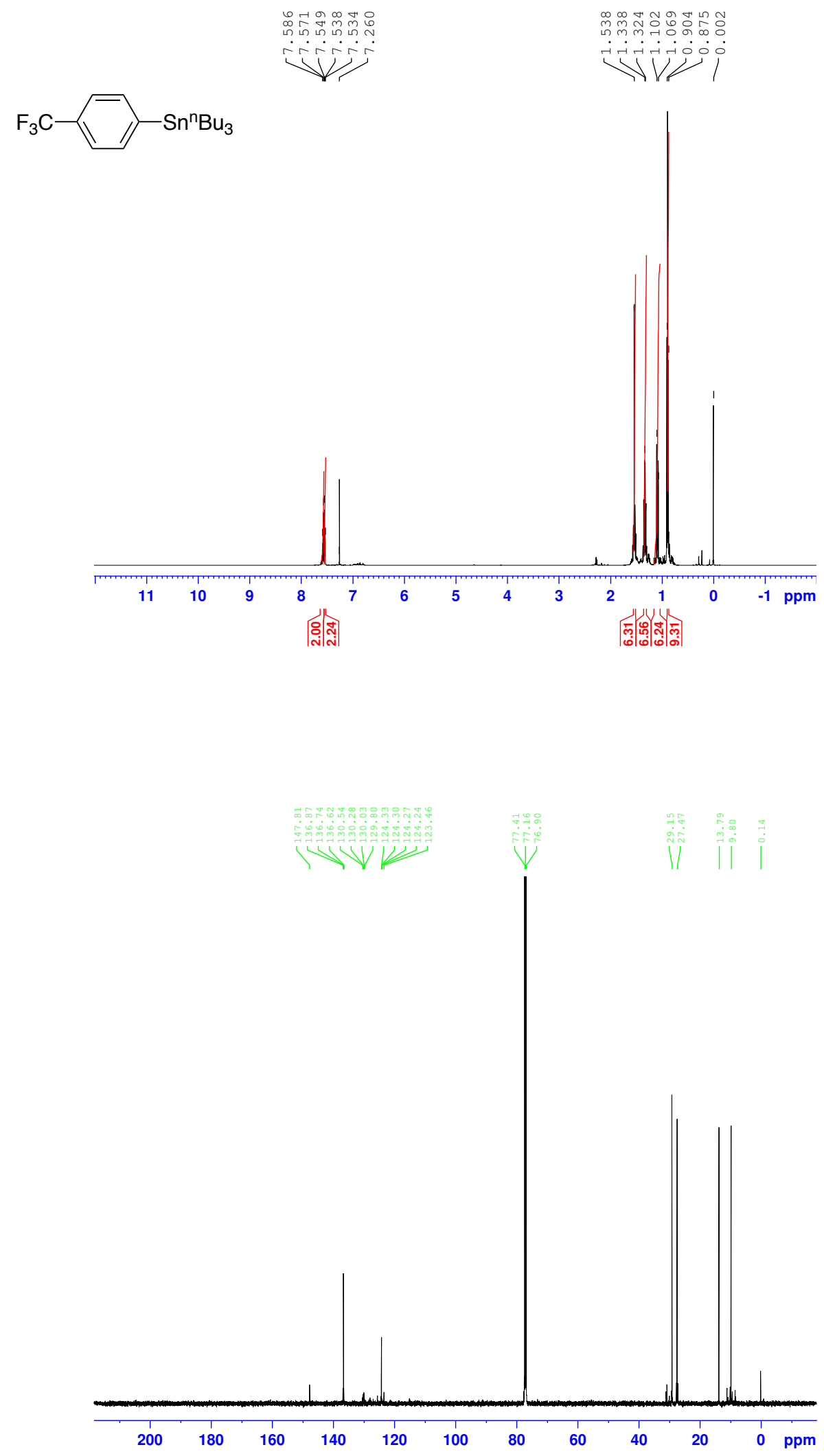

S-56 


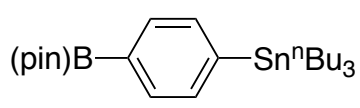

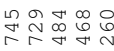

vivi

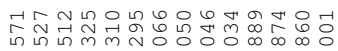
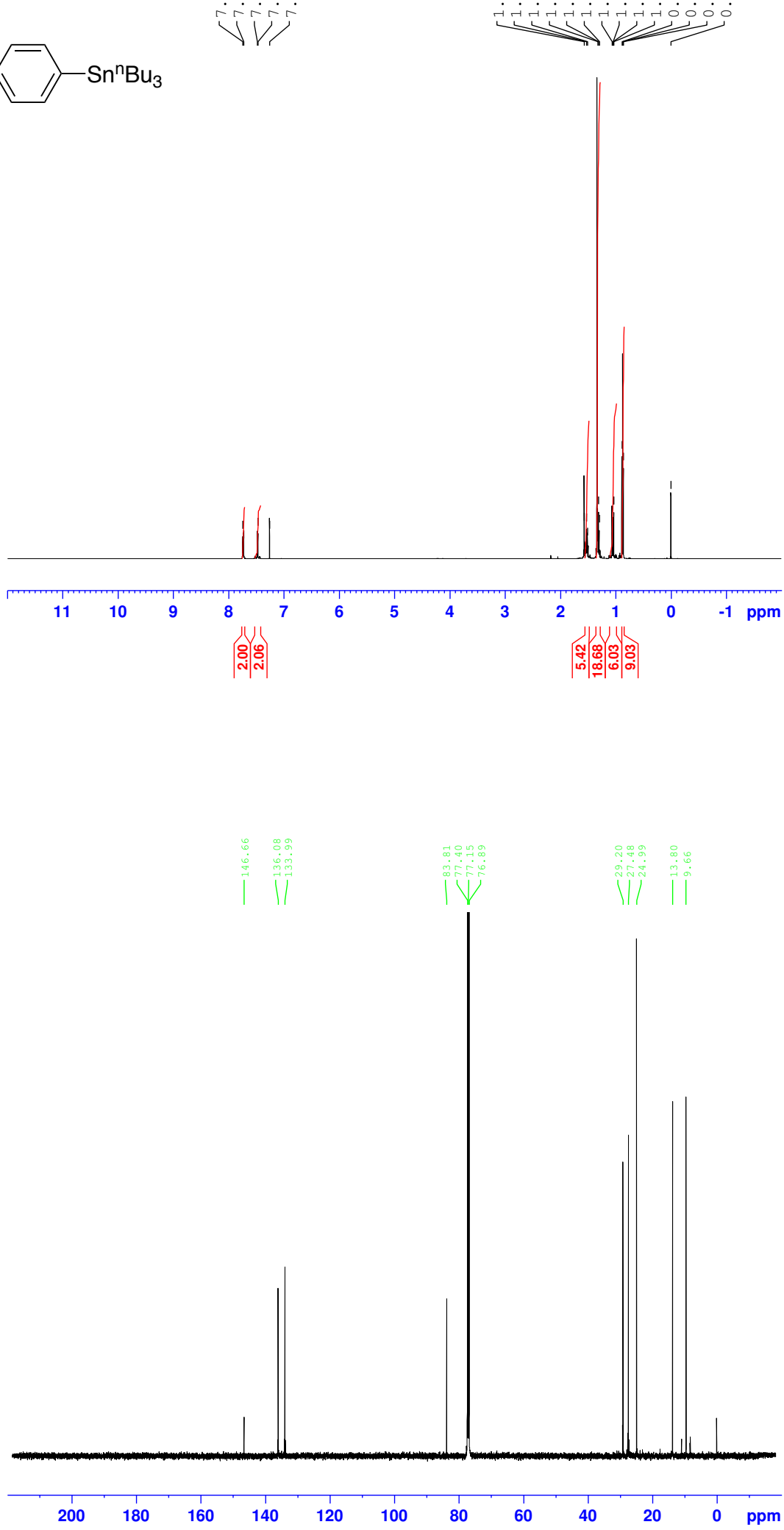

S-57 

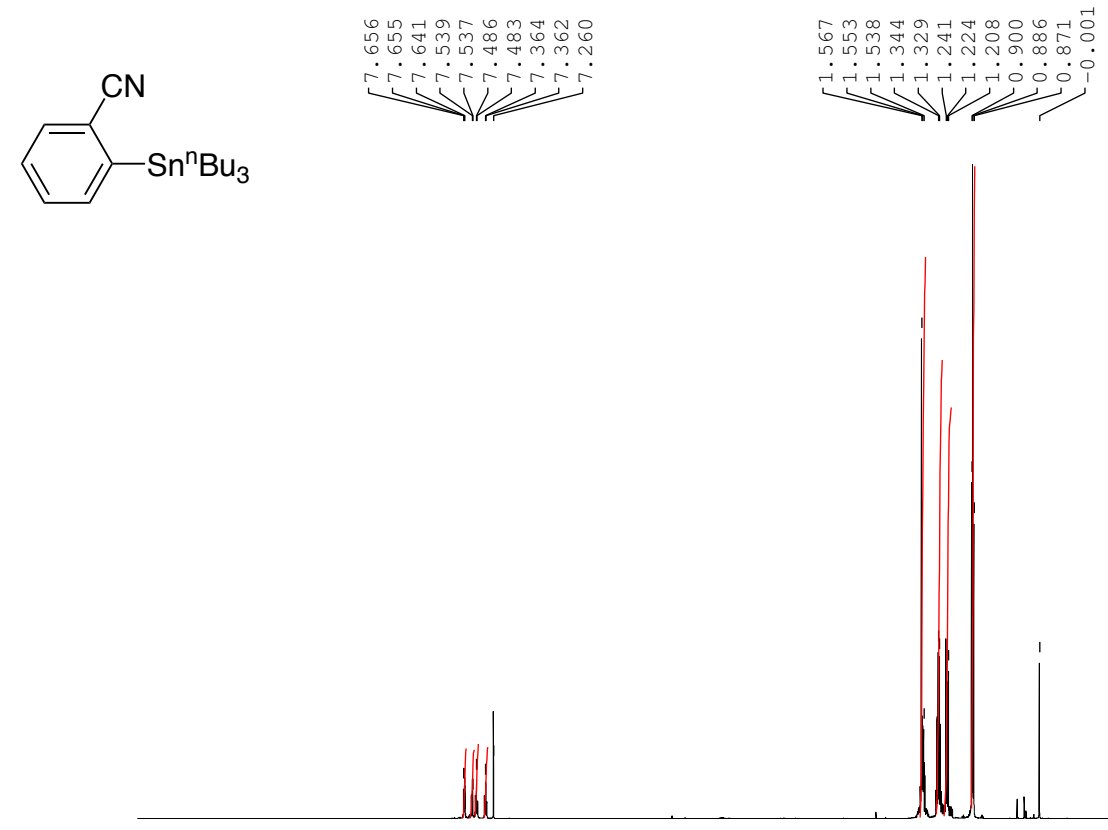

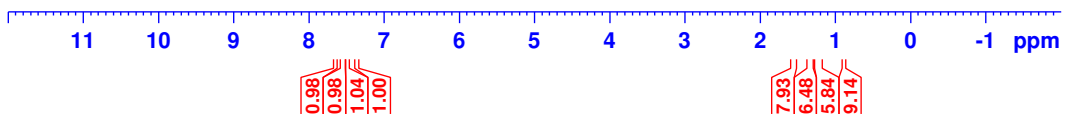

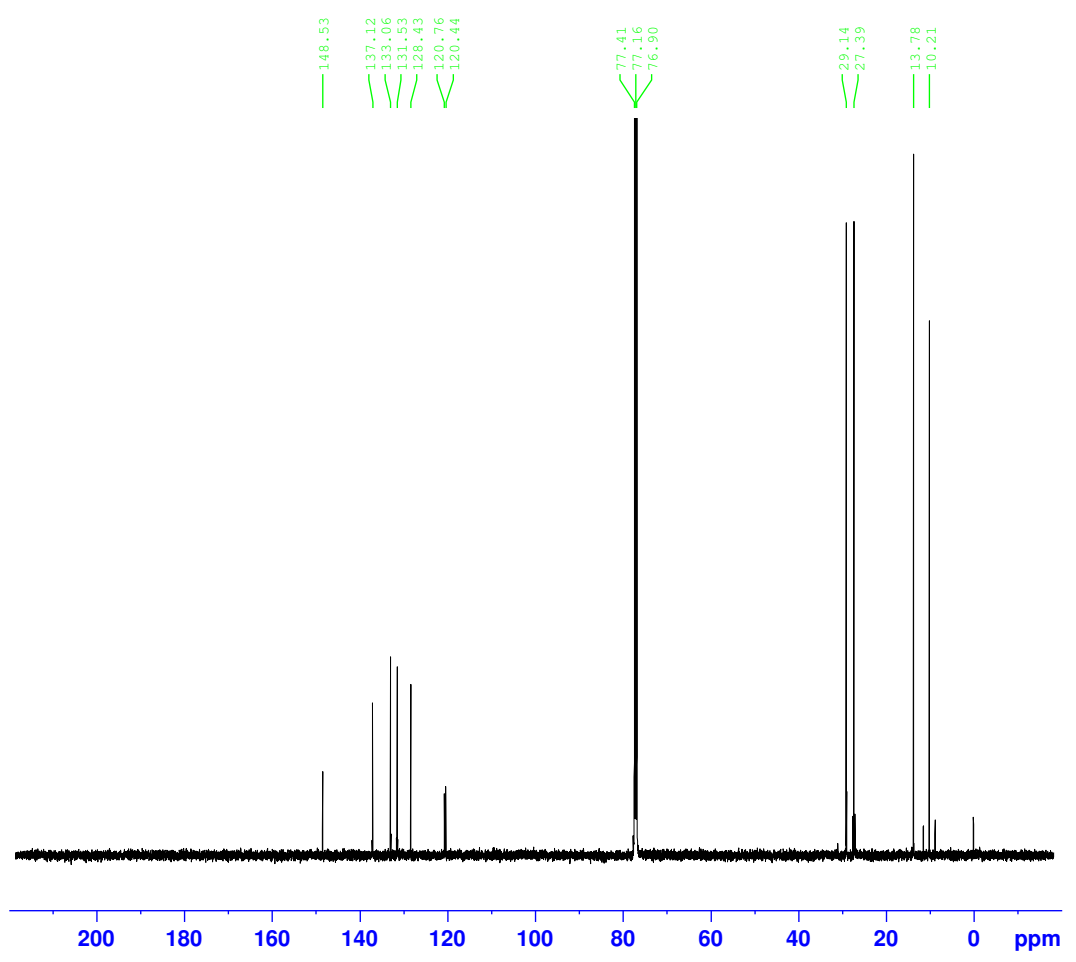

\section{S-58}



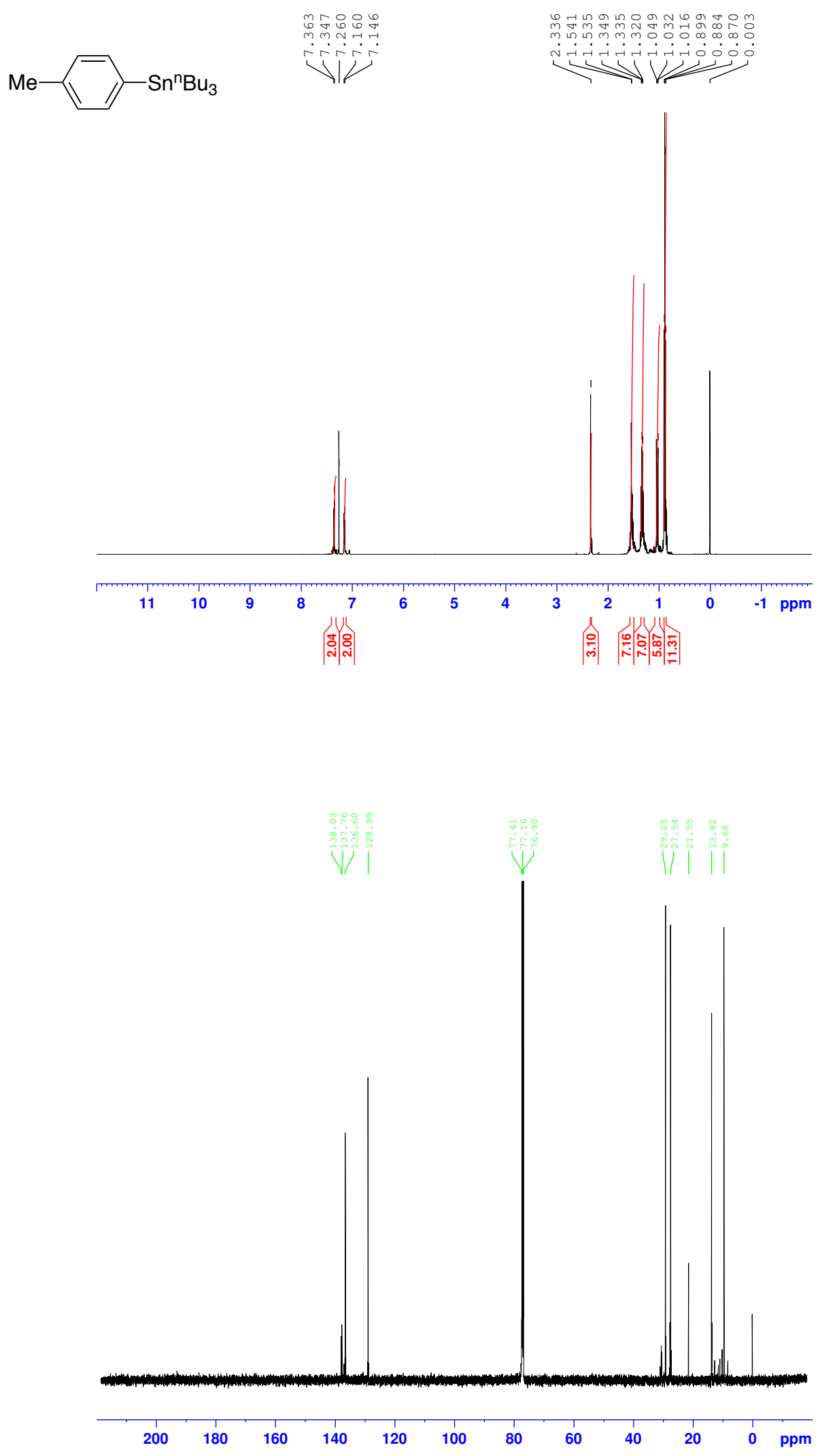

S-59 

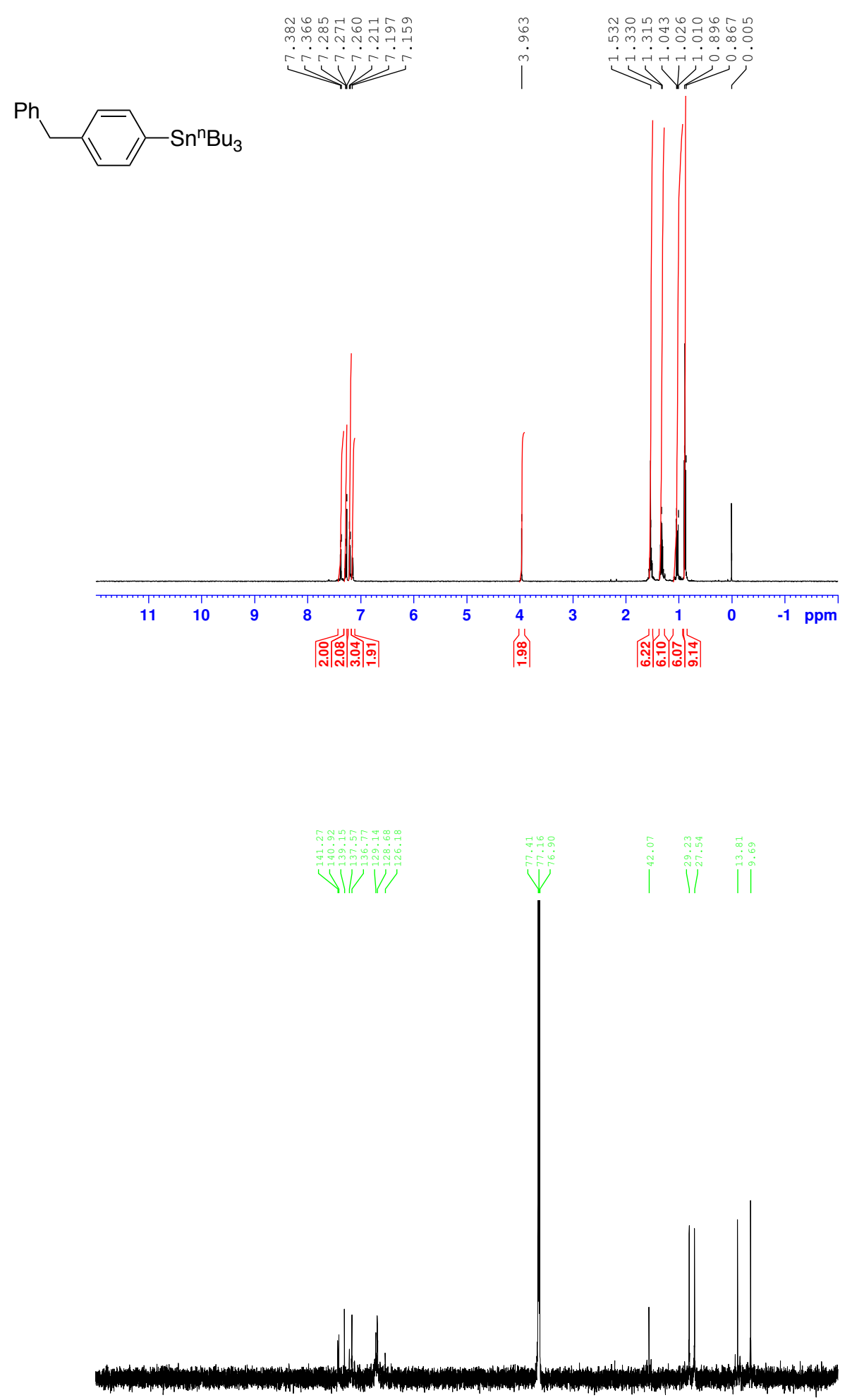

20

180

160

140

120

100

80

60

40

20

0 ppm

S-60 

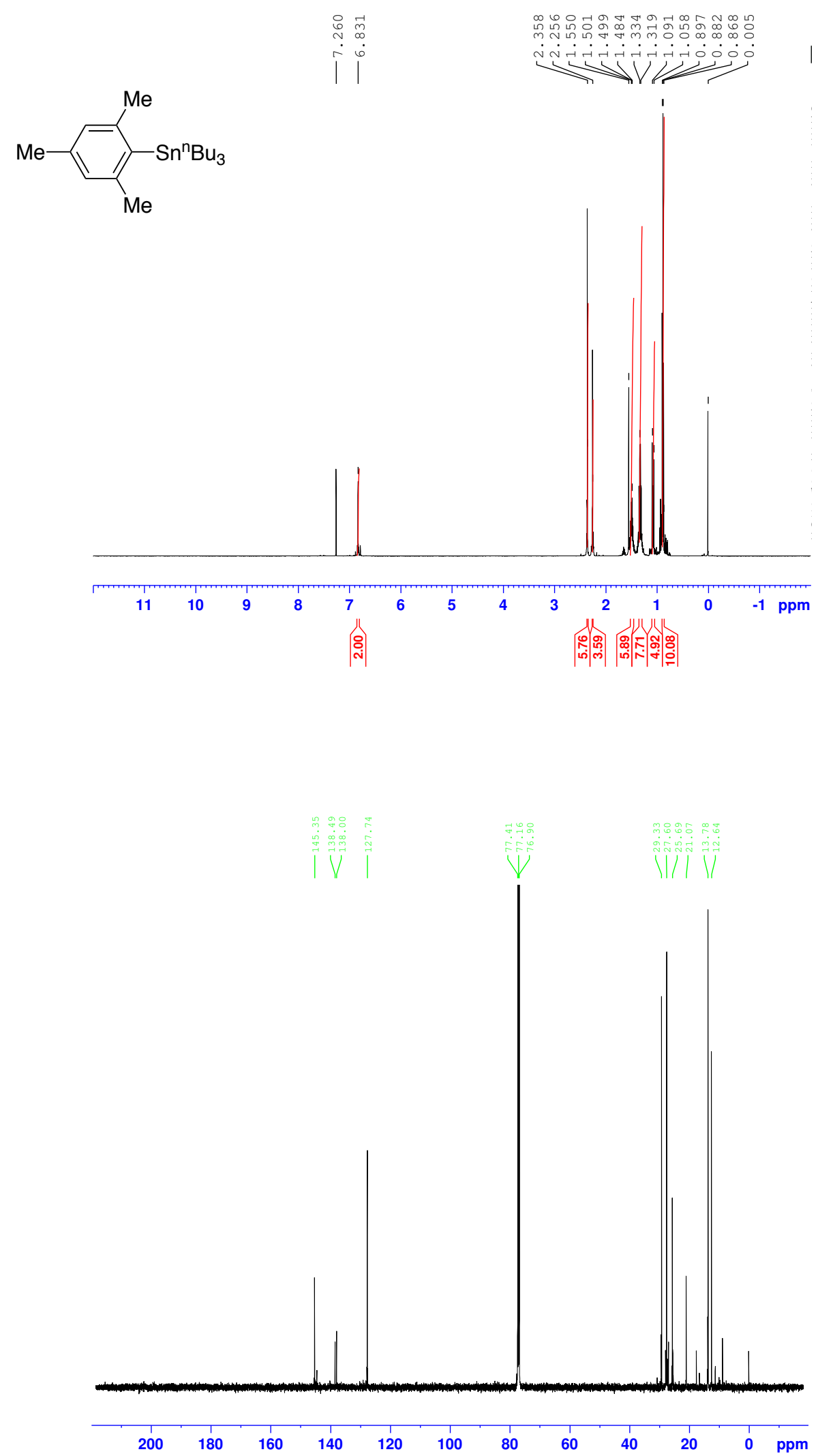

\section{S-61}



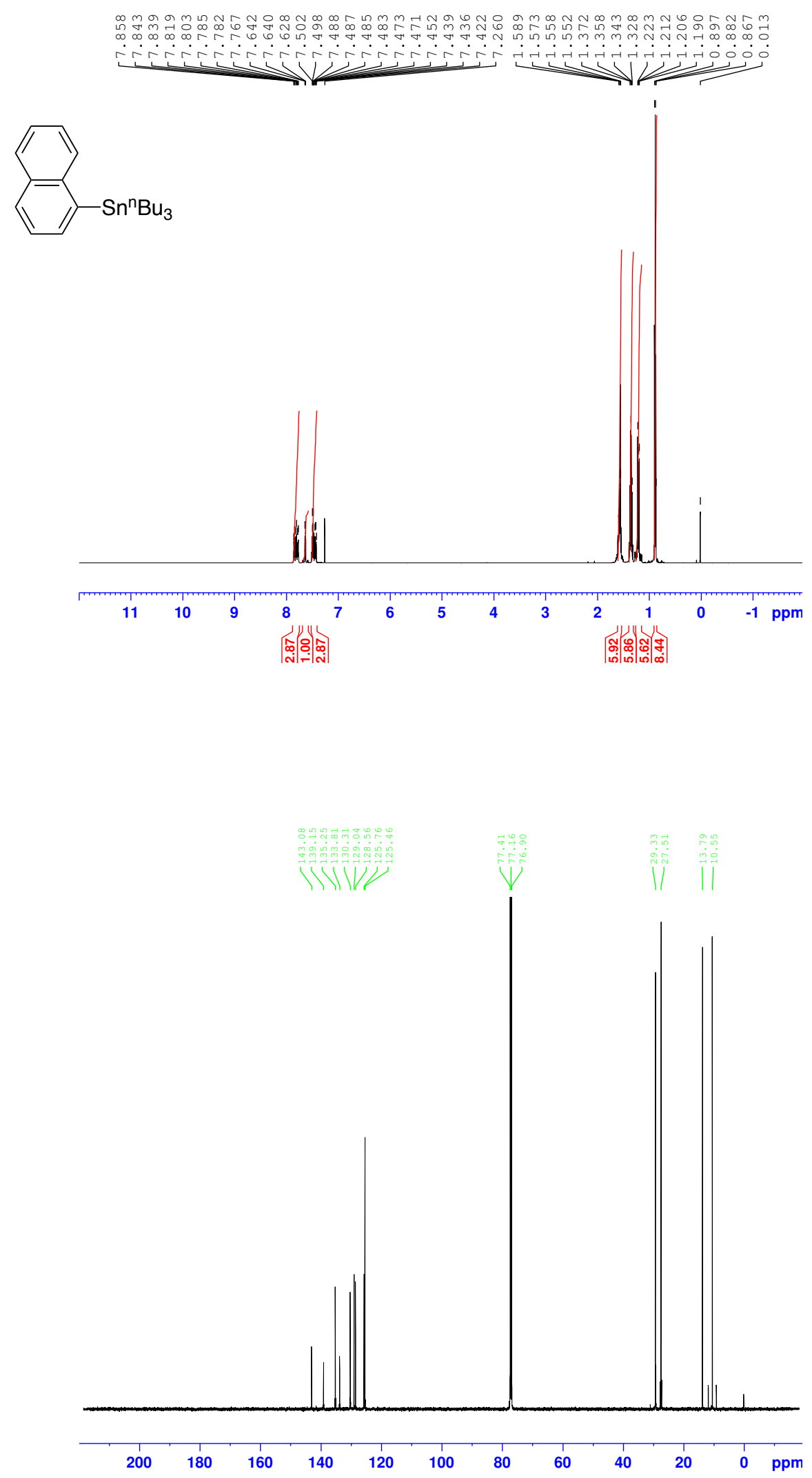

\section{S-62}



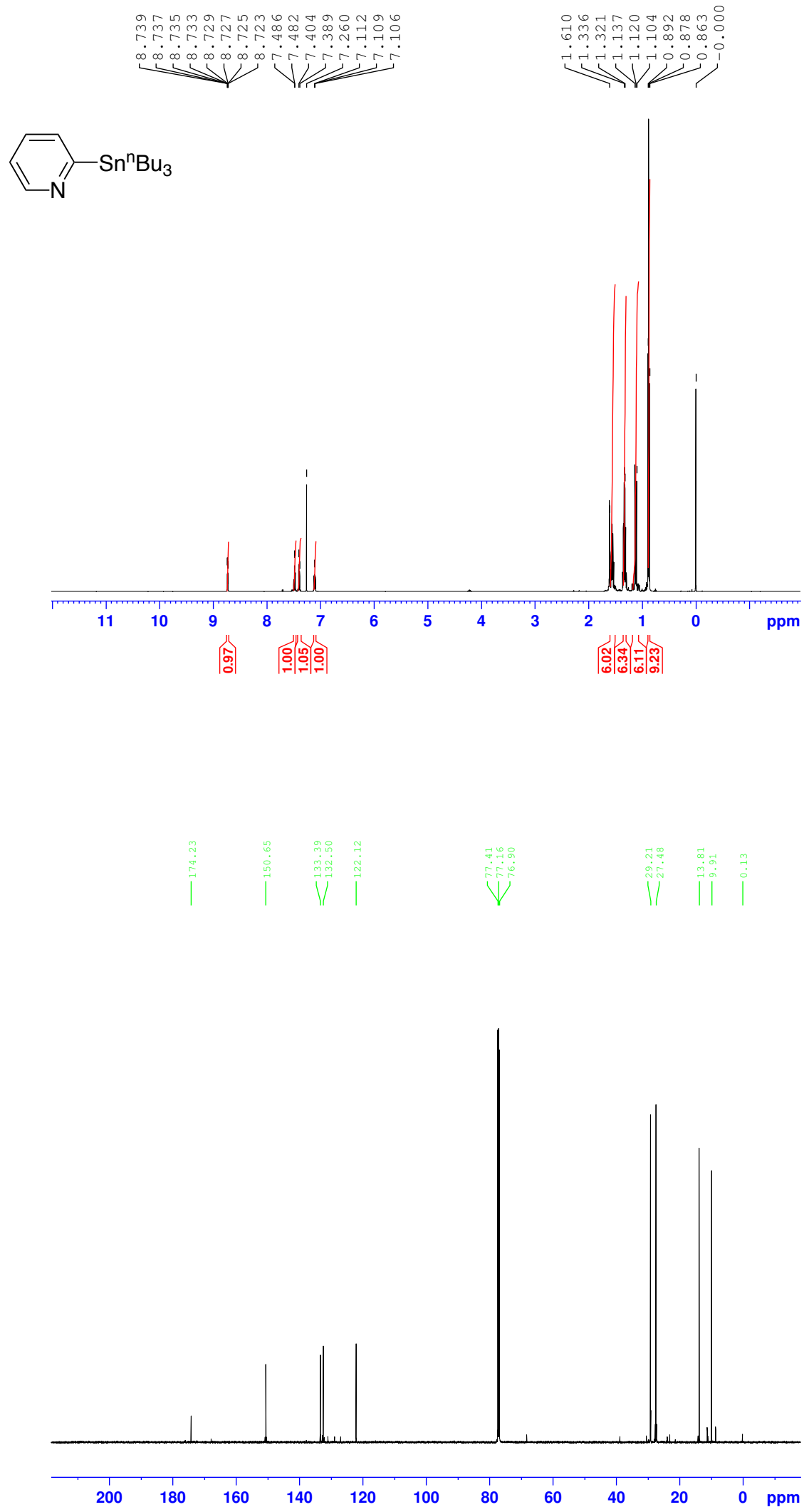

S-63 

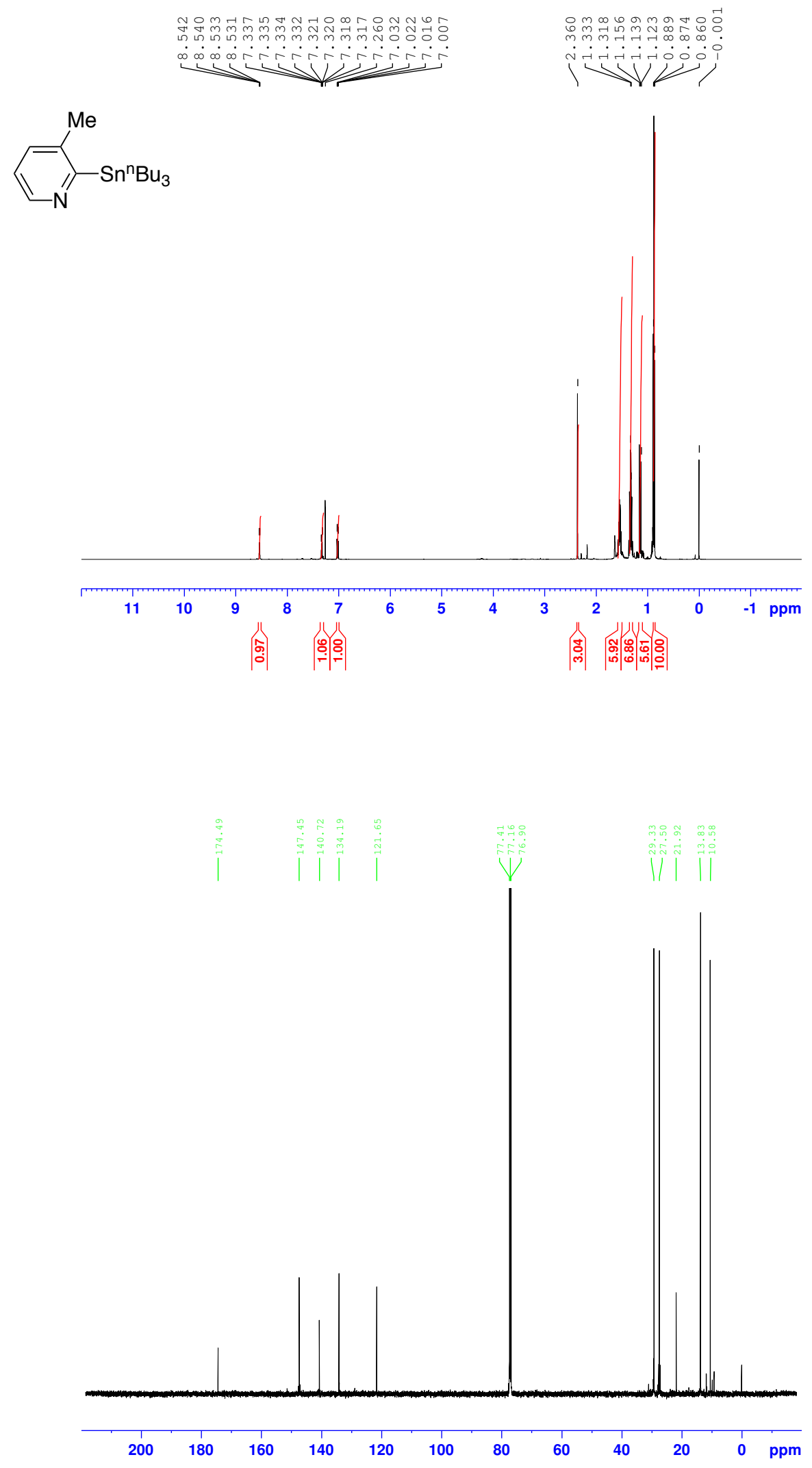

\section{S-64}



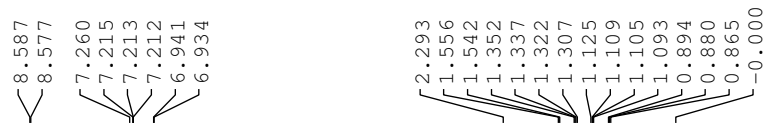

$\sum_{N}^{M e}-\mathrm{Sn}^{\mathrm{n}} \mathrm{Bu}_{3}$
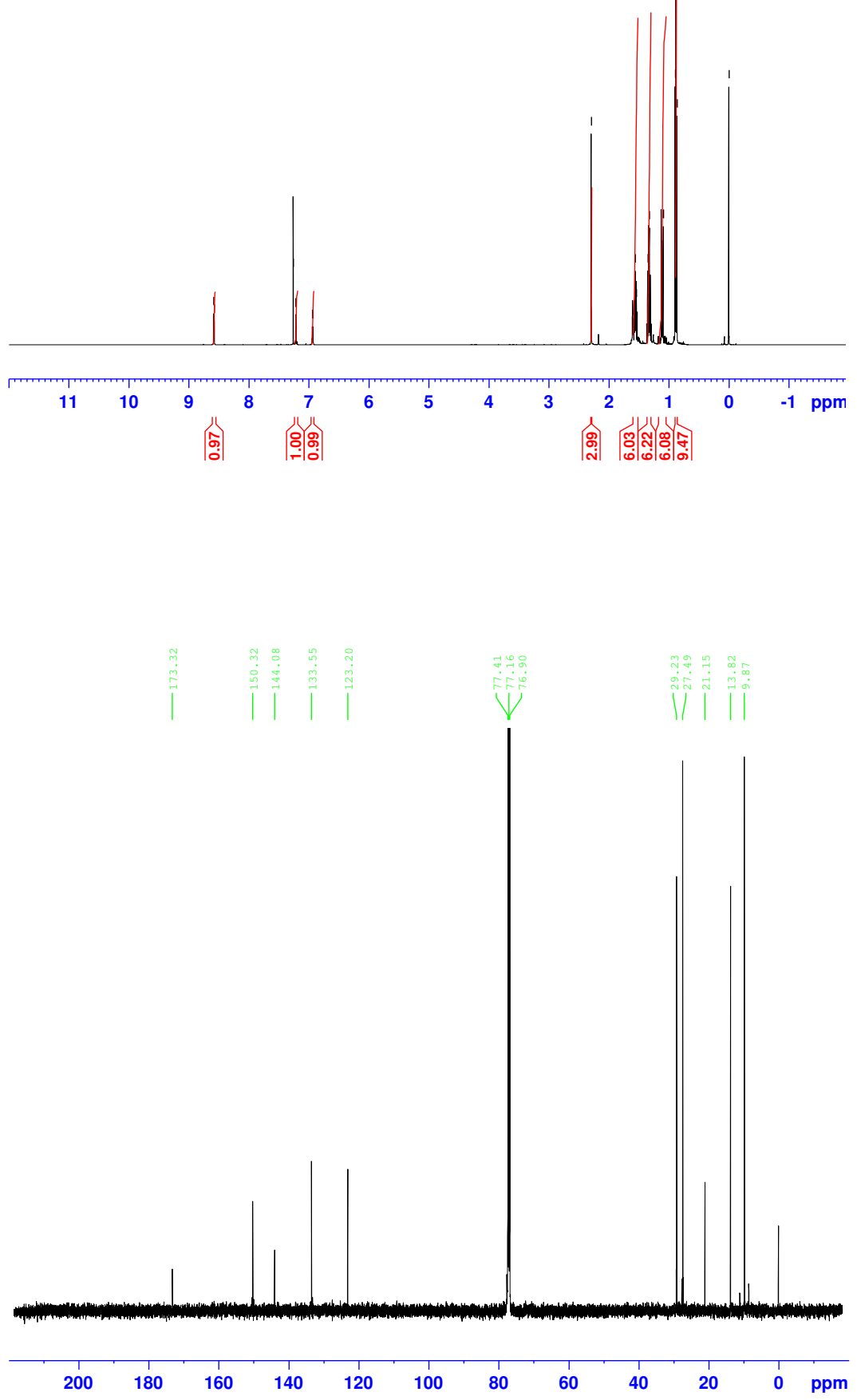

S-65 

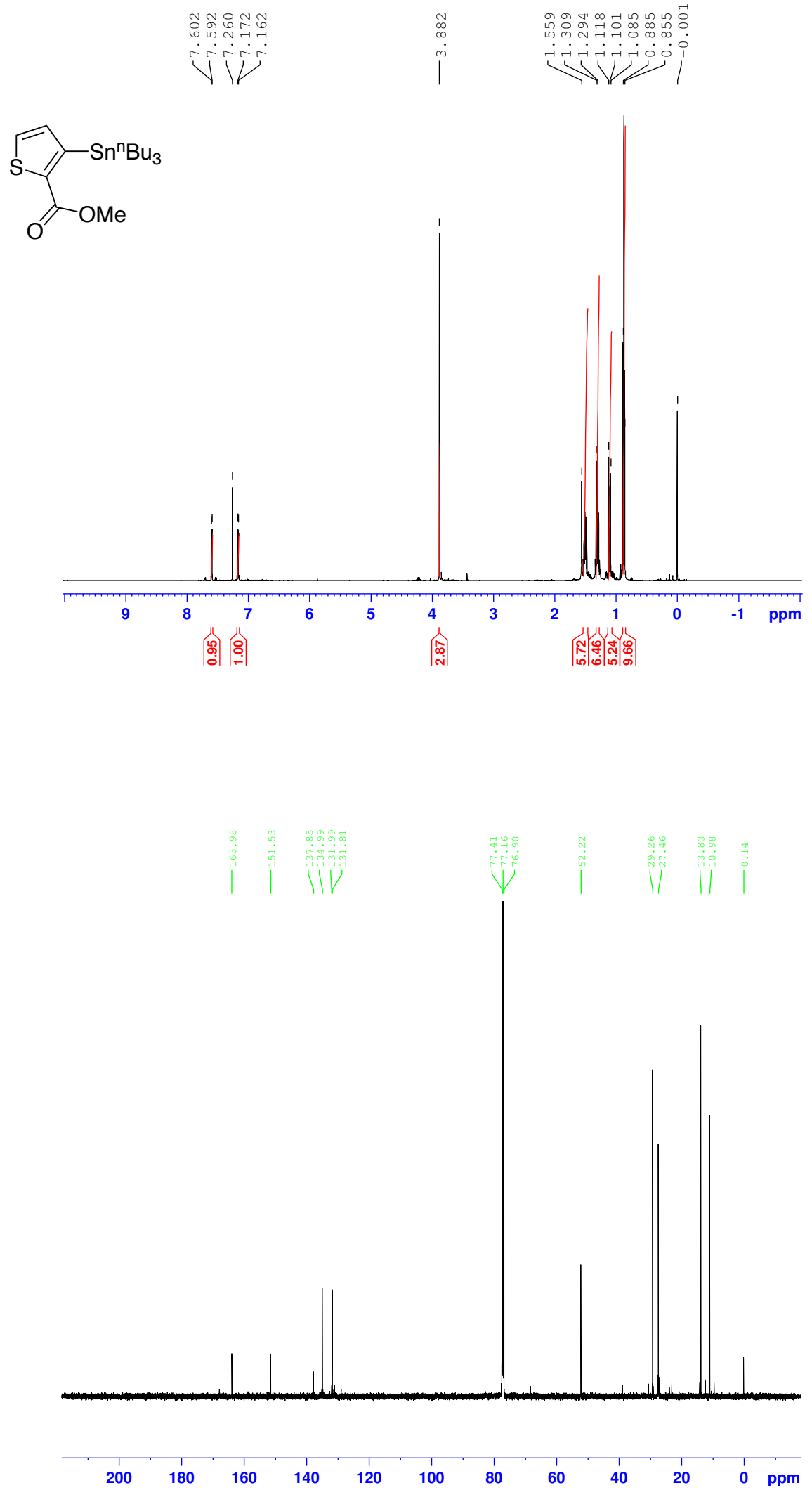

S-66 

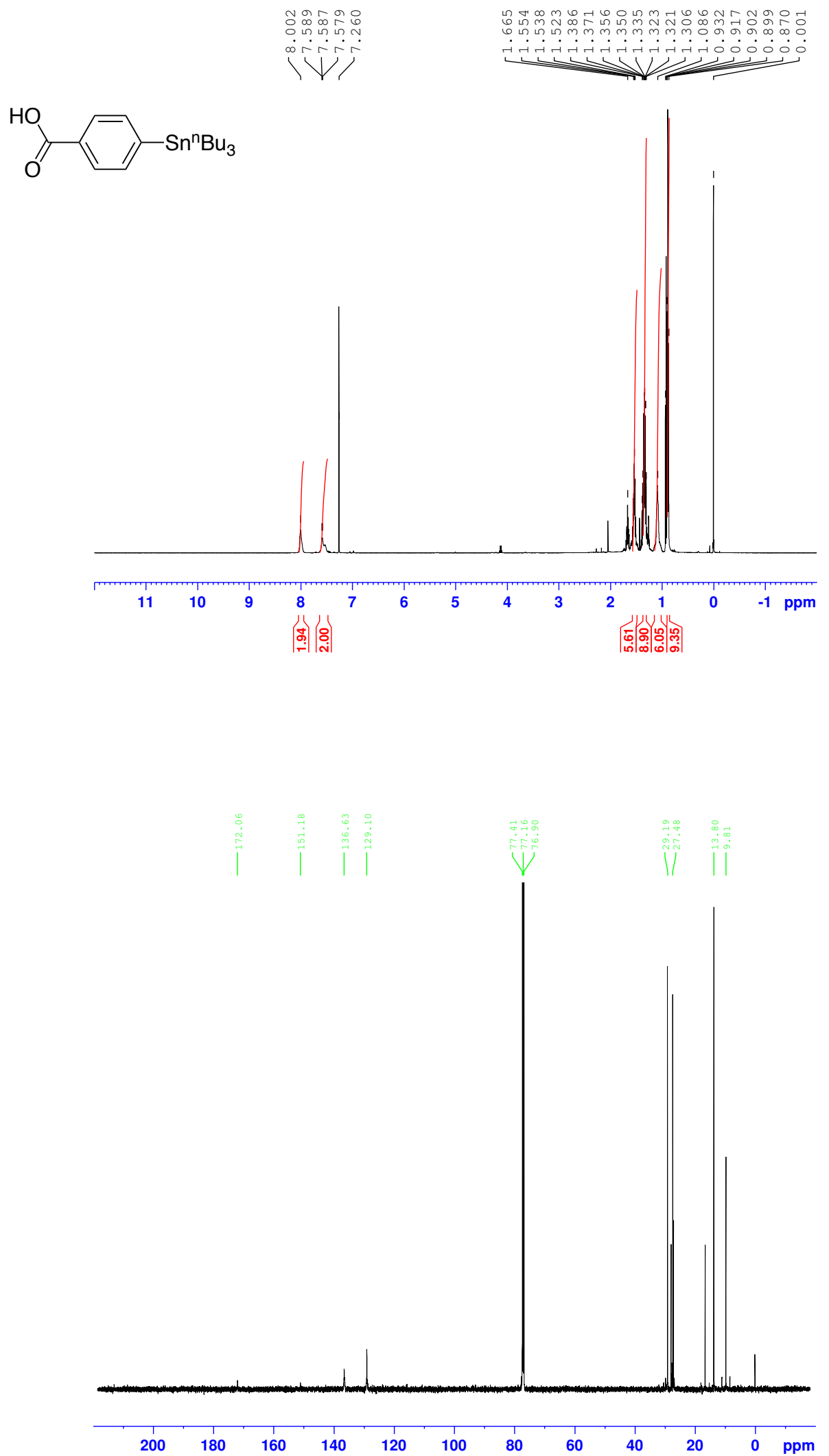

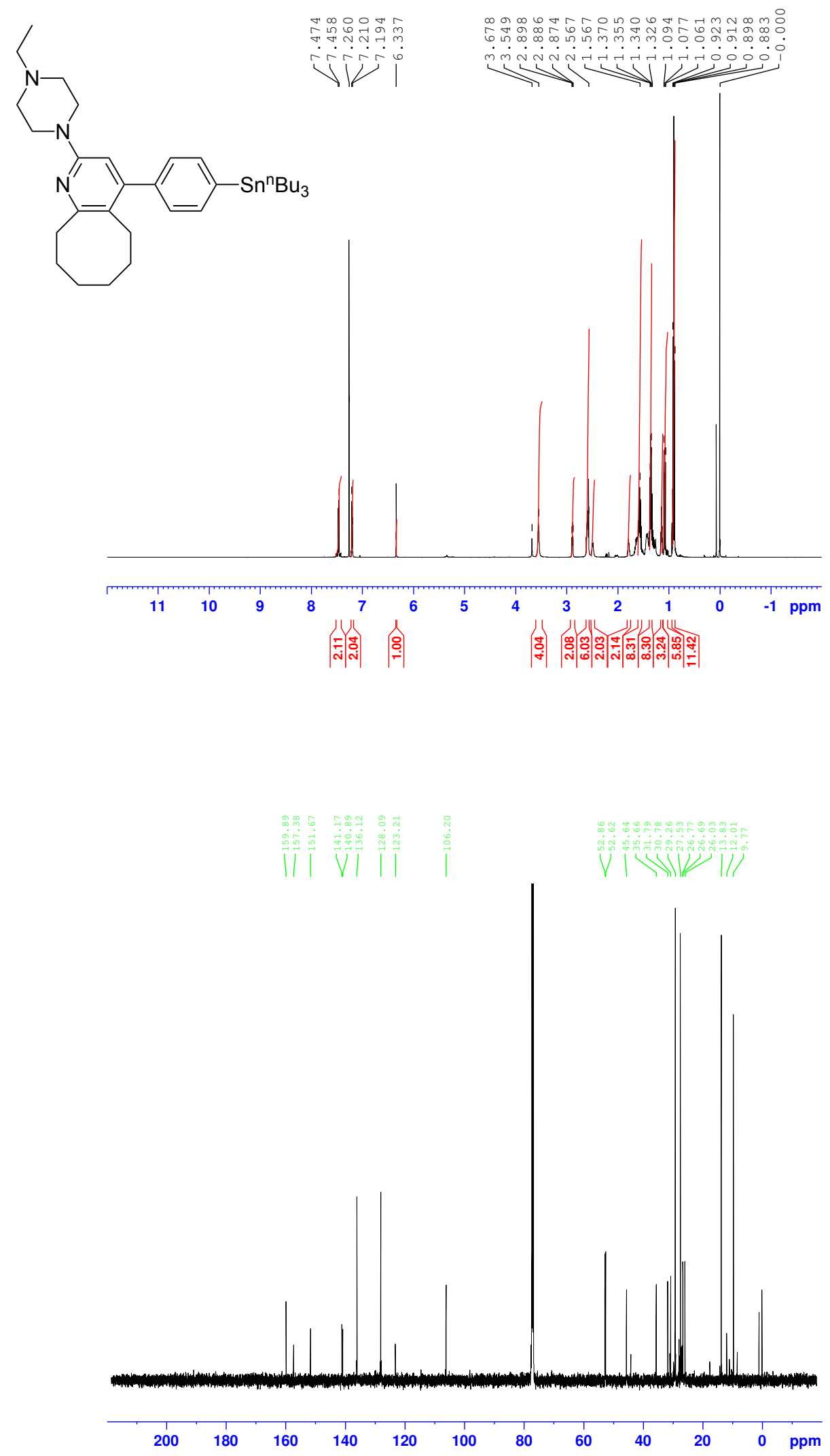

\section{S-68}



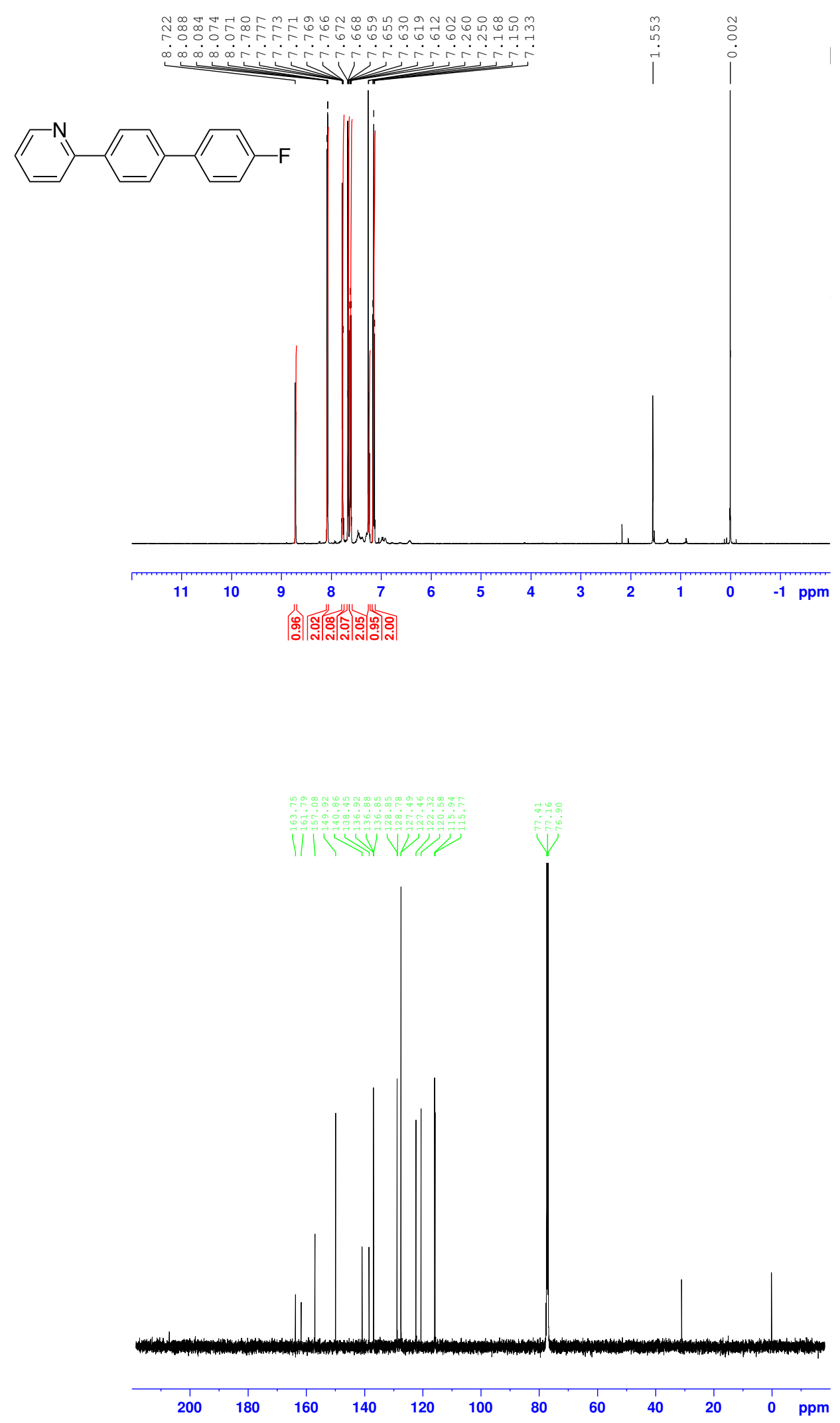

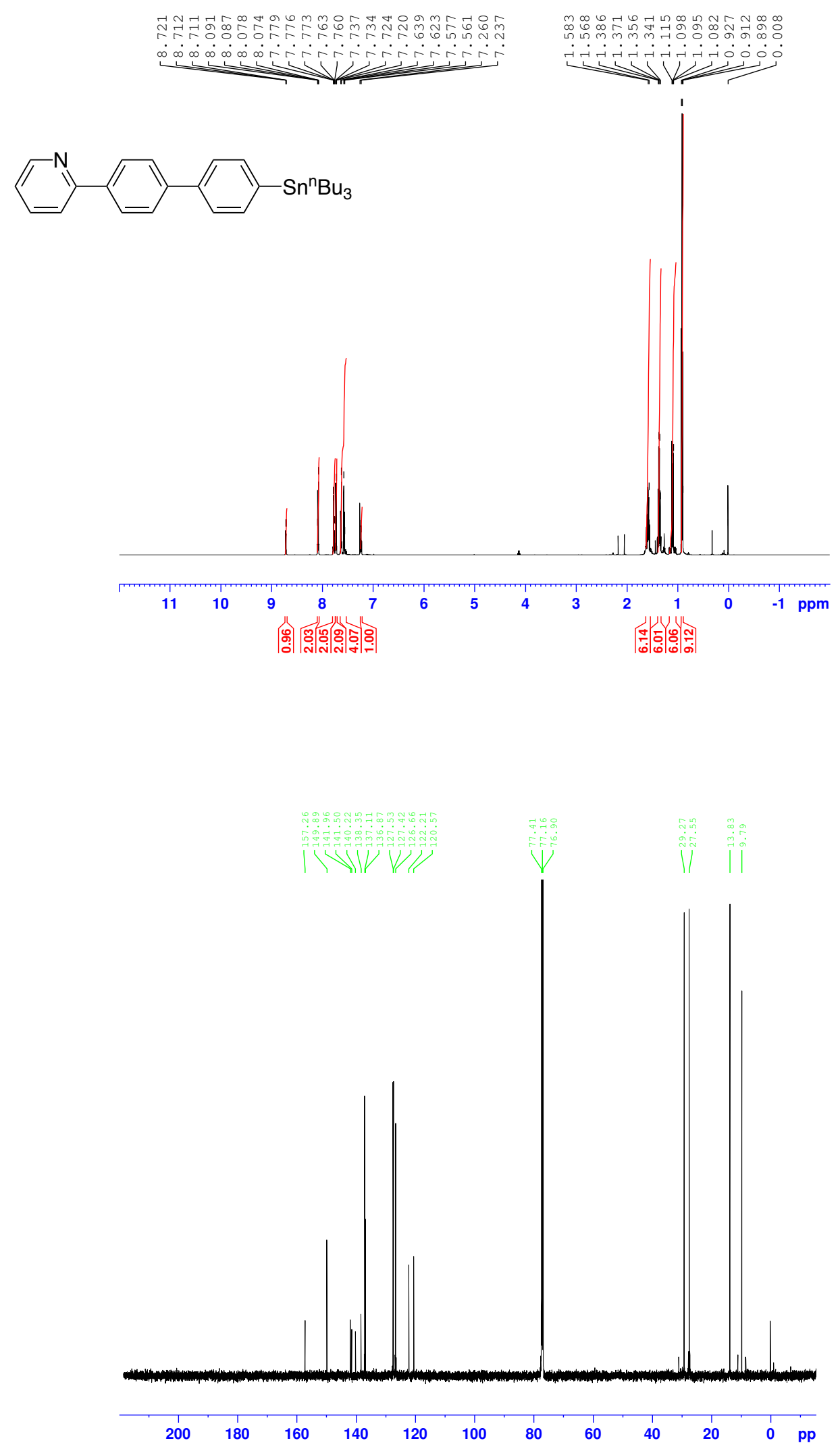

S-70 

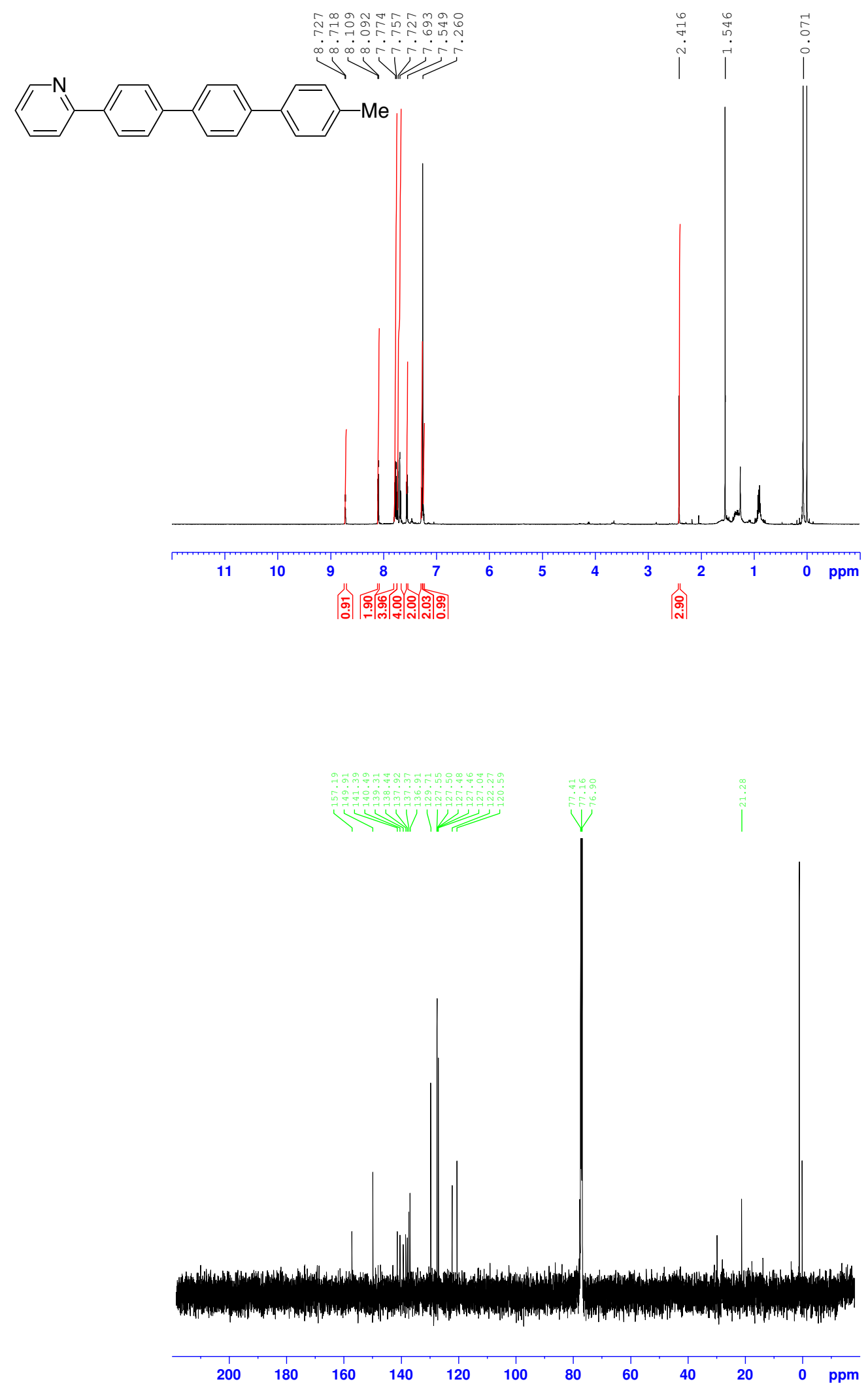

S-71 

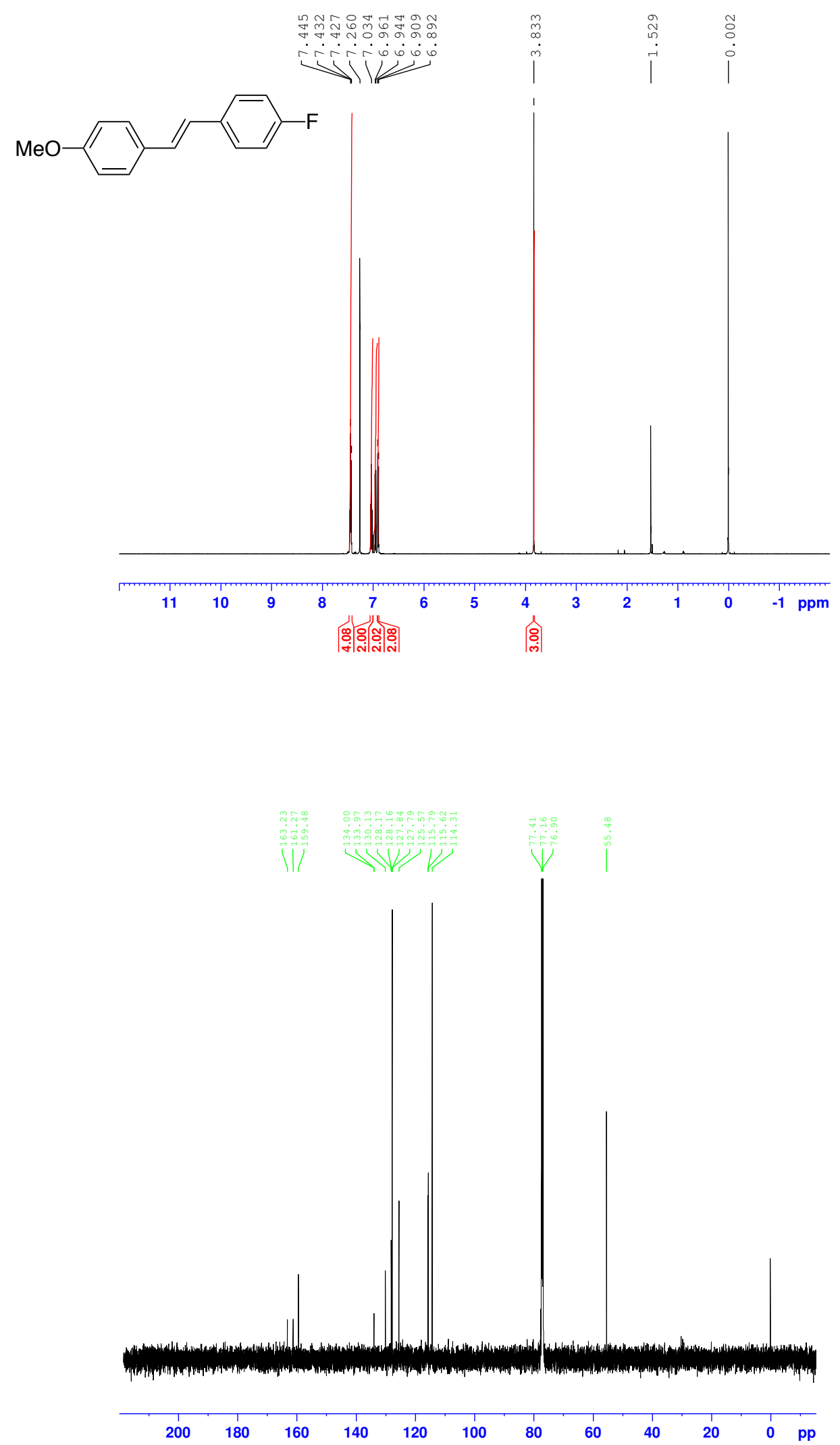

S-72 

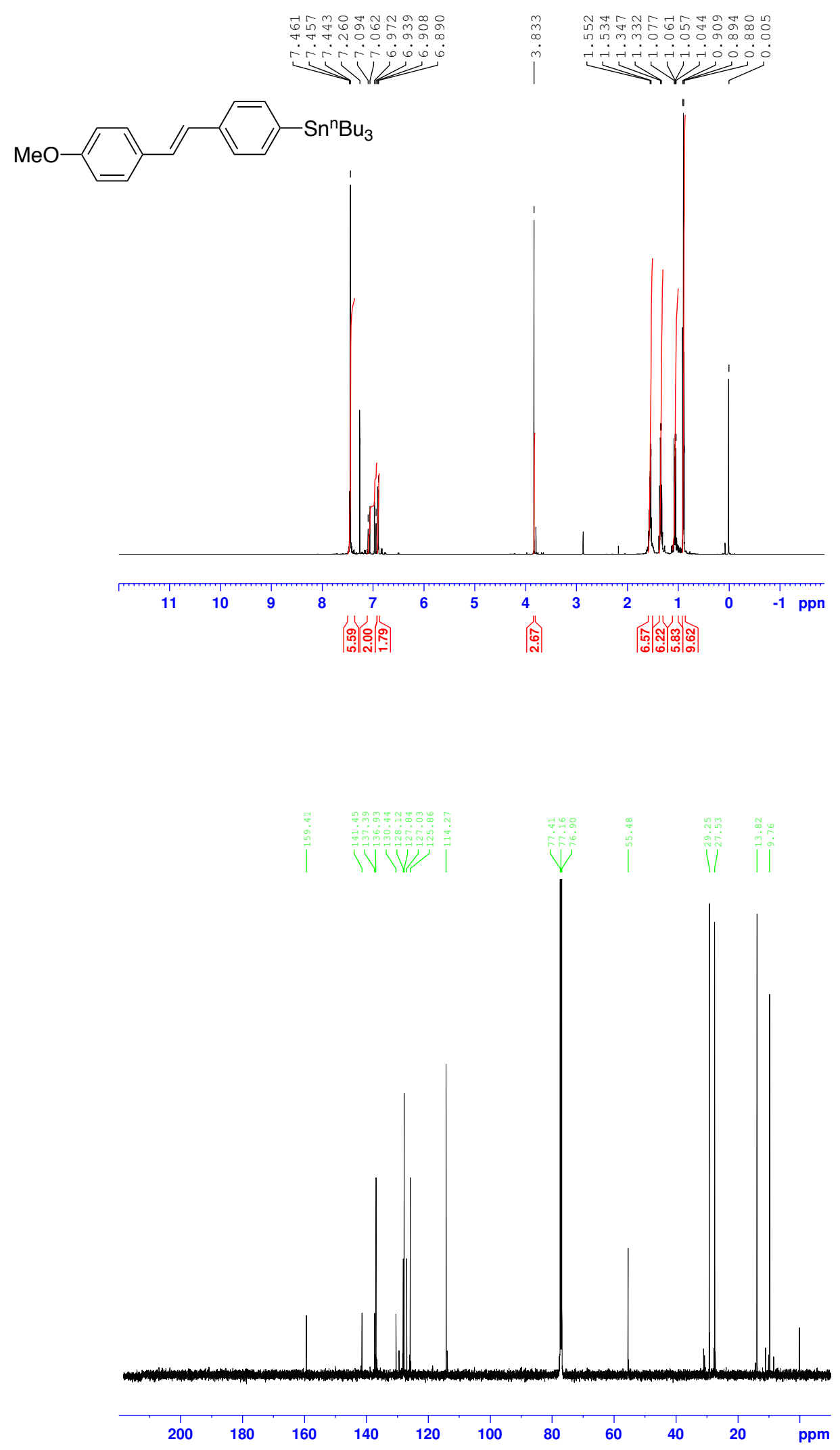

S-73 

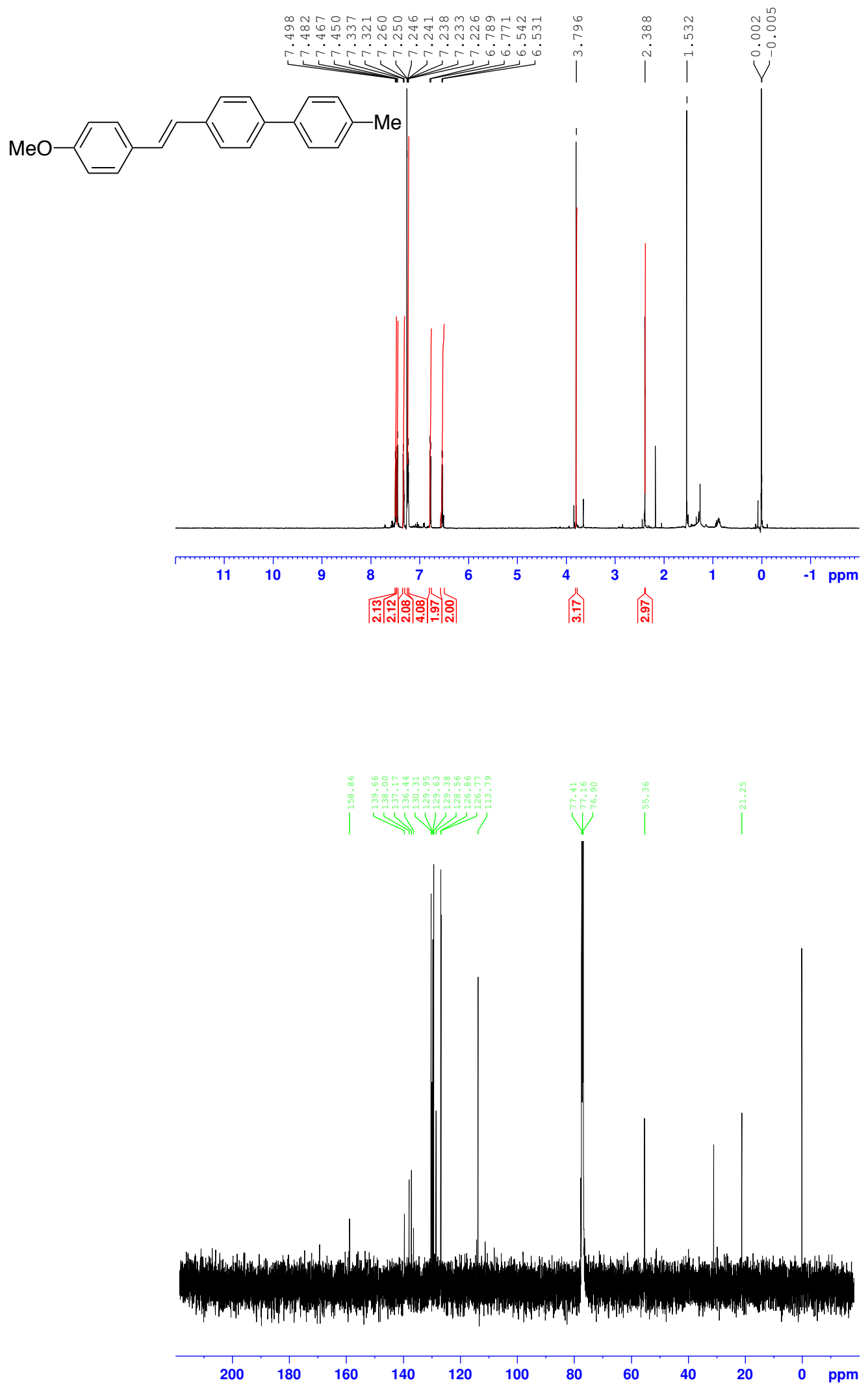

S-74 